\title{
Branched Kissing Loops for the Construction of Diverse RNA Homooligomeric Nanostructures
}

Di Liu, Cody Geary, Gang Chen, Yaming Shao, Mo Li, Chengde Mao, Ebbe S. Andersen, Joseph A. Piccirilli, Paul W. K. Rothemund, Yossi Weizmann

Submitted date: 13/03/2019 - Posted date: 14/03/2019

Licence: CC BY-NC-ND 4.0

Citation information: Liu, Di; Geary, Cody; Chen, Gang; Shao, Yaming; Li, Mo; Mao, Chengde; et al. (2019): Branched Kissing Loops for the Construction of Diverse RNA Homooligomeric Nanostructures. ChemRxiv. Preprint.

This study presents a robust homooligomeric self-assembly system based on RNA and DNA tiles that are folded from a single-strand of nucleic acid and assemble via a novel artificially designed branched kissing-loops (bKL) motif. By adjusting the geometry of individual tiles, we have constructed a total of 16 different structures that demonstrate our control over the curvature, torsion, and number of helices of assembled structures. Furthermore, bKL-based tiles can be assembled cotranscriptionally, and can be expressed in living bacterial cells.

File list (3)

RNA-bKL_Manuscript.pdf (5.02 MiB) view on ChemRxiv • download file view on ChemRxiv • download file 


\section{Branched kissing loops for the construction of diverse RNA homooligomeric nanostructures}

\footnotetext{
Di Liu ${ }^{1}$, Cody W. Geary ${ }^{2,3}$, Gang Chen ${ }^{1}$, Yaming Shao ${ }^{4}, \mathrm{Mo} \mathrm{Li}^{5}$, Chengde Mao ${ }^{5}$, Ebbe S. Andersen ${ }^{2}$, Joseph A. Piccirilli ${ }^{1,4}$, Paul W. K. Rothemund ${ }^{3}$, Yossi Weizmann ${ }^{1, *}$

${ }^{1}$ Department of Chemistry, the University of Chicago, Chicago, Illinois 60637, USA

${ }^{2}$ Interdisciplinary Nanoscience Center and Department of Molecular Biology and Genetics, Aarhus University, 8000 Aarhus, Denmark

${ }^{3}$ Bioengineering, Computational + Mathematical Sciences, and Computation \& Neural Systems, California Institute of Technology, Pasadena, CA 91125, USA

${ }^{4}$ Department of Biochemistry and Molecular Biology, the University of Chicago, Chicago, Illinois 60637, USA

${ }^{5}$ Department of Chemistry, Purdue University, West Lafayette, IN 47907, USA

*Correspondence to: yweizmann@uchicago.edu (Y. W.)
}

\section{Summary figure}

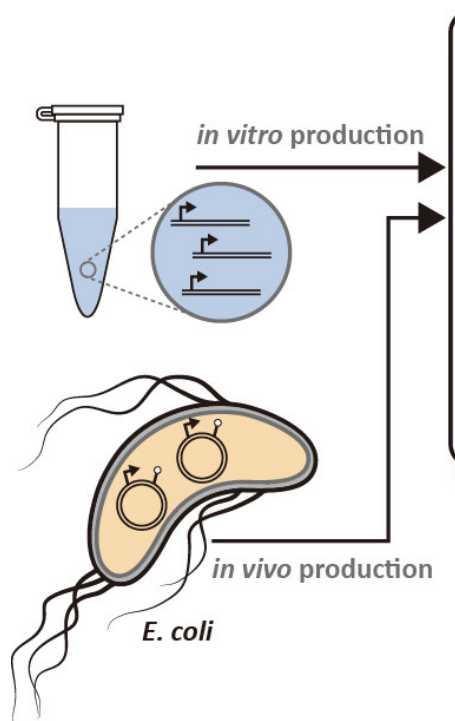

15

16 


\author{
Di Liu ${ }^{1}$, Cody W. Geary ${ }^{2,3}$, Gang Chen ${ }^{1}$, Yaming Shao ${ }^{4}, \mathrm{Mo} \mathrm{Li}^{5}$, Chengde Mao ${ }^{5}$, Ebbe S. Andersen ${ }^{2}$, Joseph A. Piccirilli ${ }^{1,4}$, Paul W. K. \\ Rothemund ${ }^{3}$, Yossi Weizmann ${ }^{1, *}$ \\ ${ }^{1}$ Department of Chemistry, the University of Chicago, Chicago, Illinois 60637, USA \\ ${ }^{2}$ Interdisciplinary Nanoscience Center and Department of Molecular Biology and Genetics, Aarhus University, 8000 Aarhus, Denmark \\ ${ }^{3}$ Bioengineering, Computer Science, and Computation and Neural Systems, California Institute of Technology, Pasadena, CA 91125, \\ USA \\ ${ }^{4}$ Department of Biochemistry and Molecular Biology, the University of Chicago, Chicago, Illinois 60637, USA \\ ${ }^{5}$ Department of Chemistry, Purdue University, West Lafayette, IN 47907, USA \\ *Correspondence to: yweizmann@uchicago.edu (Y.W.)
}

In biological systems, large and complex structures are often assembled from multiple simpler identical subunits. This strategy-homooligomerization-increases the efficiency with which a structure is genetically encoded while avoiding the problem of balancing the stoichiometry of multiple distinct units. In the design of artificial nucleic acid structures, it is similarly desirable to minimize the number of distinct subunits. Here, we present a robust self-assembly system in which homooligomerizable tiles are implemented using intramolecularly folded RNA single-strands. Cohesion between RNA tiles is specified via a novel, artificially designed branched kissing-loop (bKL) motif, which involves Watson-Crick base-pairing between the single-stranded regions of a bulged helix and a hairpin loop. By simply adjusting tile geometry, we have constructed 16 different structures that demonstrate our control over the curvature, torsion and the number of helices in linear and circular structures, as well as the ability to make finite-sized 3D cages. Minor modifications of the bKL motif enable its adaptation to DNA. Furthermore, bKL-based tiles can be self-assembled cotranscriptionally, and when inserted into a tRNA scaffold, they can be successfully overexpressed in living bacterial cells, and decorated with functional aptamers. This work both highlights the immense structural diversity that can be realized with a single-strand of RNA, and paves the way for the mass production and in vivo application of RNA nanostructures.

Large and complex structures are often required to fulfill the sophisticated functions of biological systems. One route to scale and complexity is through the expression of a single long polymer chain. Most notable is the case of the protein titin, whose largest isoform is 33,000 amino acids in length and which comprises a concatemer of 244 similar subunits ${ }^{1}$. However, a considerable fraction of large protein structures in cells are instead constructed as oligomers from many identical subunits ${ }^{2-4}$. Three primary advantages of such homooligomeric proteins have been proposed by Goodsell and Olson ${ }^{2}$, including high genetic coding efficiency, reduced 8 translation errors (i.e. incorporation of wrong amino acids and premature translation termination) and favorable assembly regulation (such as the dynamic instability and treadmilling of microtubules ${ }^{5}$ and actin filaments ${ }^{6}$ ). Reasonably, there is a length scale above which nature ceases to create finite structures whose sizes are explicitly coded by gene length (presumably about that of titin), and instead employs identical subunits to create structures whose sizes are implicitly encoded by the geometry of the subunit (for example, the geometry of a monomer controls the size of an oligomeric ring ${ }^{2}$ ), or are controlled by a system of complex regulation (for example, many different structures, of different sizes, can be made from the same subunit, as required by a particular cellular context - actin structures within the pseudopod of moving macrophage have a very different geometry than those within the muscle sarcomere ${ }^{7}$ ).

The construction of artificial homooligomeric self-assembly systems with a structural diversity that rivals those found in nature remains an important challenge for both nanoscience and chemical biology. The extraordinary programmability of nucleic acid hybridization, as demonstrated by the wide variety of sophisticated architectures created by the field of structural nucleic acid nanotechnology ${ }^{8-11}$, makes nucleic acids attractive materials for building homooligomeric systems. Moreover, natural nucleic acid-based homooligomeric systems play a number of biologically important roles, such as forming the dimer linkage structure in retroviral genomic $\mathrm{RNA}^{12}$ and creating a ring of prohead RNA (pRNA) in the packaging motor of 
$\Phi 29-$ like bacteriophages ${ }^{13}$. A better understanding of these natural systems might be reached by constructing and analyzing their artificial counterparts.

Identical subunits (also known as "tiles") for the formation of large extended structures can be created from multiple strands of $\mathrm{DNA}^{14,15}$ or RNA ${ }^{16}$. However, to prevent the formation of incomplete tiles which might act as chain terminators or create assembly defects, it is important to tightly control the stoichiometry of the different strands that make up the tiles. One way to evade this requirement is to construct each tile using a single strand. A number of systems have partly explored this approach with DNA ${ }^{17-19}$ or RNA ${ }^{20-22}$. For DNA-based systems, two difficulties have prevented the widespread design of one-stranded homooligomeric assemblies. First, despite recent advances, the high-yield synthesis of sufficiently long single-stranded DNA (ssDNA) remains relatively challenging and/or expensive, whether by phosphoramidite-based chemical synthesis, phage-based biological production ${ }^{23}$, or asymmetric PCR $^{24,25}$. Second, DNA-based designs typically employ single-stranded overhangs with free termini ("sticky ends") for cohesive interactions between subunits and, therefore, usually require multiple distinct DNA strands. Clever use of sequence symmetry can partly solve both difficulties and, in previous DNA-based systems ${ }^{17-19}$, structures were efficiently assembled from DNA tiles formed by two identical copies of a short chemically-synthesized strand containing one or more palindromic segments. However, the geometries that can be accessed using such sequence-symmetry tricks seem limited.

Compared with DNA, RNA has more appealing features such as its versatile functional capacity and its facile in vivo synthesis, making RNA nanotechnology an excitingly emerging field ${ }^{11,26}$. Partly guided by the philosophy developed in DNA nanotechnology, a wide assortment of RNA constructs, including topological structures $^{27,28}$, polyhedra ${ }^{29}$, tile-based assemblies ${ }^{16}$ and origami-type structures ${ }^{22,30,31}$, have been created. More importantly, unlike DNA, natural RNAs have an enormous variety of naturally occurring structural primitives that can be mined to greatly increase the geometric and architectural diversity of artificial nanostructures. As a result, the utilization of natural RNA folds (such as tRNA ${ }^{32}$ and pRNA $^{33,34}$ ) and motifs (such as bulges ${ }^{35,36}$, $\mathrm{K}_{\text {-turn }}{ }^{37}$, junctions ${ }^{38}$ and kissing-loop (KL) ${ }^{39}$ ) have provided the basis for a strain of RNA nanotechnology known as RNA tectonics or tectoRNA ${ }^{40-42}$, which emphasizes the combination of the unique geometries of multiple natural folds/motifs to arrive at a desired structure. Practiced in its most complex form, RNA tectonics has enabled the cotranscriptional production of arbitrary shapes out of RNA including a heart ${ }^{42}$.

Meanwhile, RNA has been used to build a number of homooligomeric assembly systems by taking advantage of two facts: (1) large quantities of very long single-stranded RNA (ssRNA) can be easily obtained by in vitro transcription from readily accessible double-stranded DNA (dsDNA) template; and (2) naturally occurring RNA motifs, such as the tetraloop-receptor ${ }^{21}$ and $\mathrm{KLs}^{20,22,43,44}$ interactions, can provide the cohesions necessary for the oligomerization without depending on sticky ends. Nevertheless, the small vocabulary of geometries or sequences available to natural motifs has limited the diversity of homooligomeric systems.

In this work, we present a novel homooligomeric self-assembly system based on an artificially designed T-shaped branched kissing-loop (bKL) motif. This bKL motif is formed via the programmable Watson-Crick base-pairings between the single-stranded regions from a bulged helix and a hairpin loop, both of which are topologically closed parts, and therefore is the so-called paranemic cohesion ${ }^{45,46}$. Importantly, this property allows the design of RNA tiles that can be associated independent of sticky ends, enabling each of the homooligomerizable tiles in this work to be constructed from a single RNA strand. Through a series of straightforward modifications of the tiles, we demonstrate that a wide assortment of extended and finite-sized structures can be assembled, and their curvature, torsion and number of helices can be controlled. Having mastered the basic geometry of bKL-based RNA tiles, we show that they can be inserted into a tRNA scaffold for overexpression in living bacterial cells, holding great promise for the mass production and in vivo applications of RNA nanostructures. Finally, we extend the use of the bKL motif in other contexts by using it to position aptamers in RNA origami tiles, as well as by adapting this motif to DNA.

\section{Results and Discussion}

Design of the bKL motif. Design of the bKL motif was inspired by a naturally occurring structure-the RNA KL complex from the HIV-1 dimerization initiation site (DIS) ${ }^{47}$. This KL is comprised of two hairpins, binding to each other via Watson-Crick base-pairings over a region of six continuous nucleotides within the 9-nucleotide 
(nt) apical loop. The remaining three nucleotides in each loop are unpaired purines (here adenines), with two positioned on the 5' side and one on the 3' side of the binding region. The two 5' adenines, serving as a linker, return the strand from the Watson-Crick binding domain to the stem and span a distance of $\sim 14 \AA$. We found that this distance can accommodate the replacement of the two-adenine linker with an RNA A-helix (Supplementary Fig. 1). This enabled us to design the bKL motif through the use of motif fusion (Figs. 1a and b), which turns one hairpin loop of the KL complex into an internal loop (or bulge). The new motif still maintains the Watson-Crick base-pairings, now formed between the complementary 6-nt regions of the bulge and the remaining hairpin loop (Fig. 1c). Consequently, as in the original KL, the interaction in the bKL is also paranemic.

We expected the bKL motif to retain the coaxial stacking present between the stems and kissing-loop region in the original $\mathrm{KL}$ and, therefore, to have a T-shape. Thus, the bKL motif is reminiscent of the previously reported DNA T-junction ${ }^{48}$, which involves cohesion between the single-stranded regions of a bulged helix and a 5' overhang. Our bKL motif possesses the assets of both the RNA KL and the DNA T-junction: like the RNA $\mathrm{KL}$, its paranemic interaction requires no sticky ends and thus decreases the number of unique strands required to make complex structures; like the DNA T-junction, its branched geometry and a certain degree of rigidity due to the coaxial stacking make it a versatile construction unit. Meanwhile, as is the case for both RNA KL and DNA T-junctions, the 6-nt Watson-Crick binding region of our bKL enables the programming of multiple specific binding interactions - in contrast, for example, paranemic GNRA tetraloop-receptor interactions have quite limited programmability and specificity ${ }^{49}$.

Multiple bKL motifs can be combined to create a single tile that self-assembles into higher-order structures. Figure 1d depicts how two bKL-forming motifs can be incorporated into a Z-shaped tile (Z-tile) to specify the formation of a ladder-like structure (below termed "ladder"). The two helix segments which occur between hairpin loops and bulges are dubbed "beams" and the helix segment between the two bulges is dubbed the "strut". In the assembled ladder, the extended coaxially stacked helices that comprise the beams and kissing regions are termed "rails". Below we show how geometrical diversity of higher-order structures can be achieved by adjusting the lengths of the beams and strut, or combining them with other structural motifs.

Control of torsion and symmetry. We began our study with the simplest possible Z-tile, one with beams are of equal length and expected to make a straight ladder. Assuming that bKLs are planar, the dihedral angle between adjacent Z-tiles (and hence torsion of a ladder) would be determined by the length of the beams. In general, to achieve a dihedral angle of zero, an integral number of RNA helical turns (i.e. multiples of 11 base-pairs (bp)) must be used for each repeat unit along a rail. For the kissing region of each bKL, we assumed that (as has been observed in a NMR structure of $\mathrm{KL}^{50}$ ) each unpaired 3' adenine stacks between the neighboring helices, adding a total of 2-bp equivalents (for clarity, we omit these two adenines in schematic figures). Therefore, we modeled the kissing region of each bKL as contributing a twist equivalent to an 8-bp A-form RNA helix. Consequently, in designing the tile LZB14 (Fig. 2a, see the caption of Supplementary Fig. 2 for detailed explanation of the nomenclature of the tiles in this work), we chose to set the length of both beams to $14 \mathrm{bp}$, so that the total repeat unit would be $22 \mathrm{bp}$, or two helical turns. Assembly of LZB14 by annealing predominantly resulted in ladders that closed into rings ranging in size from 40 to $130 \mathrm{~nm}$ in diameter (AFM and cryo-EM, Fig. 2b,c, Supplementary Figs. 16 and 17), though occasionally longer ladders with a length up to a micron were produced (Fig. 2d). No evidence of twisting was observed (e.g. periodic crossings of rails, described for other structures below) as expected. The predominance of rings suggests that ladders were flexible, and/or that the bKL motif has some intrinsic curvature: adjacent Z-tiles in a ladder are related by a simple translational symmetry, allowing the accumulation of any out-of-plane curvature caused by bKL geometry to encourage ladders to close into rings.

Introduction of torsion into the ladders provided one way to discourage ring formation and to generate extended ladders. To explore the effects of torsion, we designed two tiles, LZB13 and LZB15 (Fig. 2e), in which their both beams were 13 and $15 \mathrm{bp}$, respectively, to form left- and right-handed twisted ladders, respectively. By AFM (Fig. 2f,h, Supplementary Figs. 18 and 19), several micron long filaments were observed for both tiles. Cryo-EM, on the other hand, provided a way to visualize structures in their native states, without artifacts created by absorption on surface (Figs. 2g,i, Supplementary Figs. 6 and 20). While we could observe 
176 periodic crossings of the rails, the contrast of cryo-EM images was not high enough for us to determine the 177 absolute handedness of either ladder by tomography. Unlike the case for LZB14, small rings were rarely 178 observed. We suggest that the suppression of ring formation is due to a requirement that the phase of ladder 179 twist matches during ring closure-thus the addition of twist greatly decreases the probability that the two ends 180 of ladder find each other in an appropriate orientation to bind.

181 Manipulation of symmetry provided a second method to achieve extended ladders by suppressing 182 accumulated out-of-plane curvature in the original LZB14 tiles. If every other tile in the ladder is flipped, 183 bending from face-up tiles is canceled by bending from face-down ones (i.e. the so-called corrugated design ${ }^{51}$ ). 184 To achieve this, we converted the Z-tile into a C-shaped tile (C-tile) by changing the length of the strut from an 185 even number of helical half-turns to an odd number of half-turns so that the two beams point in the same 186 direction. We designed two different C-tiles according to this principle, having the strut length of either 16 or 27 bp (LCS16 and LCS27, respectively, in Fig. 2j). As expected, C-tiles assemble into extended ladders with both torsion and curvature minimized (Fig. 2k to n, Supplementary Figs. 21 to 24), though a few large (micron-scale) rings were also observed.

Interestingly, C-tile ladders sometimes associated laterally along their length into wider structures, as were observed for the LCS16 tile by AFM (Fig. 2k, Supplementary Fig. 21), but not by cryo-EM (Fig. 21, Supplementary Fig. 22). We suggest that sample preparation for dry AFM concentrates the ladders and encourages them to associate, and that the major factor promoting lateral association is base stacking between struts on different ladders. Base-stacking occurring at the blunt ends of helices presents an opportunity for the helices to stick together; this phenomenon has been employed to program the association of a variety of DNA nanostructures $^{52,53}$. For struts on different ladders to stack, the ends of the struts must experience some displacement away from the position where they connect to the beams. This implies a certain flexibility of the bKL motif (the top of the T-shape in each bKL must slip sideways, see Supplementary Fig. 7 for more details). We suggest that the appearance of stacking between C-tile ladders, but not for Z-tile ladders, is further evidence of the lack of torsion and curvature in C-tile ladders. This is because any significant bend or twist in a ladder would disrupt the long-range alignment of struts, decreasing the opportunity for their ends to match and stack. Experience from previous work on artificially designed stacking interactions ${ }^{52,53}$ supports this conclusion, as the shape of structures had to match closely for stacking to occur.

Besides the flexibility which allows the stacking of LCS16 ladders, the structures examined so far provide evidence for three additional types of intrinsic flexibility of the bKL motif (Supplementary Fig. 8). First, the broad range of ring sizes observed in LZB14 ladders implies a range of out-of-plane bending of the beams. Second, the strut must have some freedom of rotation along its axis: the same strut length (21 bp) is used for all three Z-tiles, LZB13, LZB14, and LZB15, and thus rotational flexibility of the strut is necessary to accommodate the different torsions present in three different ladders. Finally, bKL must also exhibit some in-plane bending flexibility of the beams: such in-plane bending is necessary for the formation of the LZB13 left- and LZB15 right-handed twisted ladders because it provides the curvature of the rails to follow the trajectory of a circular helix.

Control of in-plane curvature. In-plane bending of bKL can be harnessed to design closed annuli by using Z-tiles having two beams of different lengths $\boldsymbol{X}$ and $\boldsymbol{Y}$, chosen so that the binding of tiles forms trapezoidal repeat units. Ladders created by such tiles would bend toward the shorter trapezoid base so that the shorter trapezoid base defines the inner circle of an annulus and the longer one defines the outer circle. To achieve flat annuli, the bases of the trapezoid are chosen to be an integral number of helical turns in length (tiles denoted with names that end in $\boldsymbol{X} / \boldsymbol{Y}$, also see the caption of Supplementary Fig. 2 for more details).

We began our exploration with annuli of relatively low curvature. Figure 3a shows Z-tile RZB14/25 whose beam lengths differ by one helical turn, setting the repeat length along the inner circle to two helical turns (22 bp) and that of the outer circle to three helical turns $(33 \mathrm{bp})$. Geometrical analysis predicted that an unstrained planar annulus should contain 16 or 17 tiles (Supplementary Fig. 9). Accordingly, we expected annuli having a diameter of 47 or $50 \mathrm{~nm}$. By AFM (Fig. 3b, Supplementary Fig. 25) and cryo-EM (Fig. 3c, Supplementary Figs. 10 and 26), annuli of different sizes were observed, potentially due to the intrinsic flexibility of the bKL and/or the assembly kinetics (i.e. the rate of tile addition versus ring closure). Structures containing greater or fewer 
than 15-18 tiles assumed apparently nonplanar geometries in order to release strain: those with fewer tiles assumed the shape of a conical frustum, while those containing more tiles assumed saddle shapes.

Moving towards greater curvature, we designed Z-tile RZB14/36 to have a two-turn difference between longer and shorter trapezoid bases (Fig. 3d), expecting it to form smaller annuli containing 8 or 9 tiles (31 or 35 $\mathrm{nm}$ in diameter). To our surprise, two distinct structures formed (Fig. 3e, Supplementary Figs. 11 and 27). One was the expected smaller annulus, with a range of diameters from 26 to $38 \mathrm{~nm}$ (roughly 7 to 10 tiles); the other was a long linear filament, which appeared to be $\sim 30 \%$ thicker (1.6-1.8 $\mathrm{nm}$ in height) than the annulus (1.2-1.3 $\mathrm{nm}$ in height) by AFM (Supplementary Fig. 11). The height of the annulus is consistent with typical dry AFM measurements of RNA on mica. The height of the filament implies a structure having regions with two layers of RNA helices. We propose that the filaments are, in fact, partially double-layered ladders, with three rails and a narrow, triangular cross section (bottom of Fig. 3d and Supplementary Fig. 12). In the proposed structure, one edge of each filament is a single rail (and hence a single layer) formed by the shorter beam of each tile (which contributes two turns to this rail). The other edge is comprised of two closely associated rails (and hence two layers), either of which is formed by the longer beam of every other tile (which contributes four turns to either rail). The resultant pairing of two short beams for each long beam yields a four-turn repeat unit with no tendency for in-plane bending. We note that if a purer sample of small annuli is desired, the size difference between the two products enables efficient purification. Passing the reaction mixture through a $0.22 \mu \mathrm{m}$ filter removes most of the filaments, leaving annuli as the dominant species in the filtrate (Fig. 3f,g, Supplementary Figs. 28 and 29), though a small amount of short fragments and circularized filaments still remain.

The formation of two different structures from RZB14/36 suggested that the large in-plane curvature of 8-tile RZB14/36 annuli might be near the bending limit that can be achieved with such bKL-based Z-tiles. To investigate this limit, we designed RZB14/47 to have a three-turn difference between the longer and shorter beams (Fig. 3h) for the even smaller annulus (5- or 6-tile, and 25 or $29 \mathrm{~nm}$ in diameter). If the annulus were indeed formed with five tiles, its inner circle would be a 110-bp double-stranded RNA (dsRNA) circle, with a diameter of $\sim 10 \mathrm{~nm}$, which is close to the diameter $(\sim 11 \mathrm{~nm})$ of the dsDNA loop around the nucleosome, and near the lower size limit for synthesis of double-stranded DNA (dsDNA) circles $^{54}$ without using special bent sequences or DNA-binding proteins to enhance curvature. With a persistence length $(64 \mathrm{~nm})$ longer than that of dsDNA $(52 \mathrm{~nm})$, dsRNA is stiffer and so it was unsurprising that no single-layered small annuli were observed experimentally. Instead, analogous to the filaments formed with RZB14/36, we found only double-layered annuli (Fig. 3i, Supplementary Fig. 30). In these double-layered annuli, the pairing of two short beams (contributing four turns) with a single long beam (contributing five turns) results in a repeat unit with a one-turn length difference, creating the gentle bend (also see Supplementary Fig. 13).

Combining torsion and curvature. All the above structures were either essentially linear, or curves confined to the plane. In the case of the LZB13 and LZB15 ladders, though the rails trace out a double helix, the curves traced by the center of those ladders were still approximately a straight line. By incorporating both torsion and curvature into a tile, true 3D space curves can be achieved. Starting from the annulus-forming tile RZB14/25, left- or right-handed torsion was introduced by simultaneously removing or adding one bp from both beams to produce tiles RZB13/24 and RZB15/26, respectively (Fig. 3j). The introduction of torsion discouraged the closure of the assembled filaments, and thus the majority of structures were helical ladders which took on a ramen-noodle-like appearance. Compared to the left-handed nano-ramen assembled from RZB13/24 (Fig. 3k,1, Supplementary Figs. 31 and 32), right-handed RZB15/26 nano-ramen appeared to be more flexible, as suggested by an increase in the formation of cyclic structures (Fig. 3m,n, Supplementary Figs. 33 and 34). The interplay of twist, curvature and some bKL flexibility makes it difficult to predict the helical parameters of nano-ramen. Experimentally, we estimated the diameter of RZB13/24 nano-ramens to be $16-20 \mathrm{~nm}$ and the period to be $\sim 55 \mathrm{~nm}$; RZB15/26 structures trace out a somewhat wider helix with a diameter of 20-30 nm and a period of $\sim 60 \mathrm{~nm}$.

5 Multi-railed ladders. The addition of more rails to a ladder presents an opportunity to create stiffer filaments, of different symmetries, and with more opportunities for functionalization-we explored two approaches to the design of tiles that would form multi-railed ladders. The first approach employs Z-tiles with beams of unequal 
278 lengths, so that the repeat unit formed using the longer beam (plus kissing domain) has an integral number of 279 full helical turns $L$, and the repeat unit formed using the shorter beam (plus kissing domain) has a possibly 280 non-integer turns $S$, which approximately divides $L$ to yield an integer $M$. The resulting ladder has a central rail 281 comprising the shorter beams and $M$ peripheral rails comprising the longer beams, and the peripheral rails are 282 spaced at intervals of $\theta=360^{\circ} / X$ around the central rail, where $X$ is the smallest integer such that $S X$ is 283 approximately an integer. This approach is essentially similar to the unintended three-railed ladder assembled 284 from the previous RZB14/36 tile (Fig. 3d). However, for that structure, $L=4$ turns, $S=2$ turns, $M=2, X=1, \theta$ $285=360^{\circ}$; thus, were the tile not flexible, the two long-repeat rails would be superimposed on each other, yielding 286 a steric clash.

287 To create a three-railed ladder, we designed RZB19/47 (Fig. 4a, also see Supplementary Figs. 35 and 36), 288 for which $L=5$ turns, $S=27 \mathrm{bp} \approx 2.5$ turns, $M=2, X=2$, and $\theta=180^{\circ}$, by placing the two long-repeat rails on 289 opposite sides of the central rail. The overall helicity of the assembled structure is because that the distances $2902 S=54 \mathrm{bp}$ (along the central rail) and $L=55 \mathrm{bp}$ (along a peripheral rail) differ by a single base pair over a 291 five-turn span. To create an analogous four-railed ladder, we designed RZB10/47 (Fig. 4b, also see 292 Supplementary Figs. 37 and 38), for which $L=5$ turns, $S=18 \mathrm{bp} \approx 5 / 3$ turns, $M=3, X=3$, and $\theta=120^{\circ}$, so that

302 Out-of-plane curvature for 3D nanocages. The structures that we have so far described have no exactly well-defined size: they were either extended ladders, capable of reaching potentially arbitrary length, or annuli with a relatively broad distribution of diameters. In the latter case, though the curvature of tiles was designed to specify annuli with a particular number of tiles, $N$, because $N$ was relatively large, inclusion of an extra tile or omission of a tile required only a small structural deformation (per subunit), which could be easily accommodated by the flexibility of the tiles.

In contrast, it is common for natural proteins to homooligomerize into finite assemblies with a precisely defined number of subunits. Such systems exhibit closed point group symmetries that constrain the number of subunits arranging around a principle axis of rotation ${ }^{2}$. We implemented such a strategy in designing tile CZB12b11 so that it could preferentially form a $13.3 \mathrm{~nm}$ wide tetrameric cage (Fig. 5a). Beginning with a standard Z-tile, we introduced an out-of-plane bend into each beam by inserting at its midpoint a $90^{\circ}$-angle 5 -nt bulge (AACUA) motif ${ }^{5}$. This bulge motif, from domain Ila of the internal ribosome entry site (IRES) of the hepatitis $\mathrm{C}$ virus $(\mathrm{HCV})$, has been previously used to construct RNA nanosquares ${ }^{35}$ and nanoprisms ${ }^{56}$. Here, equipped with this motif, the original Z-tile was transformed into a 5-helix segment geometry. We arranged the pattern of bKL sequence interactions (see the numeric bKL labels in Fig. 5a) in such a way that the resulting tetramer was specified to have $\mathrm{D}_{2}$ symmetry. This arrangement ensured that only even-numbered $N$-mers of $\mathrm{D}_{N / 2}$ symmetry would be allowed to prevent the likely formation of the 3-fold symmetric trimers and the 5-fold symmetric pentamers due to the flexibility of Z-tile. The formation of dimers and hexamers, though allowed by symmetry, requires greater flexibility and distortion of the tiles and thereby is unlikely.

Beyond theoretical considerations of geometry and symmetry, experimental salt conditions can exert profound effects on the outcome of self-assembly - the formation of specific motifs, such as $\mathrm{KL}^{20}$, and overall RNA tertiary structure ${ }^{57}$ are highly dependent on $\mathrm{Mg}^{2+}$ concentration. Therefore, we optimized conditions for tetrameric nanocage formation as a function of salt, and found that an annealing buffer containing $100 \mathrm{mM} \mathrm{Na}^{+}$ and $0.3 \mathrm{mM} \mathrm{Mg}^{2+}$ (Fig. 5b, lane 3 ) gave the desired product in excellent ( $>95 \%$ ) yield. Visualization by AFM revealed square-shaped particles of uniform size (Fig. 5c). Cryo-EM analysis (Fig. 5d) enabled single-particle reconstruction (Fig. 5e), verifying the gross features of the tetramer cage (a width of $13.7 \mathrm{~nm}$ ). The reconstructed suggests that struts assume a $\sim 10^{\circ}$ tilt, conferring an overall left-handed twist to the cage. Though 
329 a handful of RNA nanocages have been previously constructed using multiple tiles ${ }^{32,33}$ or using a single tile 330 formed from multiple RNA strands ${ }^{56}$, to the best of our knowledge, ours is the first example of a 331 homooligomeric nanocage assembled from only one strand of RNA and with a high yield.

332

333 Cotranscriptional assembly and cellular production. For all the structures described so far, tiles were first 334 purified from an in vitro transcription and then self-assembled via annealing (slow cooling from $70{ }^{\circ} \mathrm{C}$ to $4{ }^{\circ} \mathrm{C}$ 335 over the course of 2.5 hours). However, the intramolecular folding of RNA secondary structure (10-100 $\mu$ s) is 336 expected to proceed several orders of magnitude faster ${ }^{58,59}$ than both the tiles' synthesis by RNA polymerase $337(\sim 0.5 \mathrm{~s}$ at $200-400 \mathrm{nt} / \mathrm{s})$ and the tiles' intermolecular assembly via bKL $\left(\mathrm{t}_{1 / 2}=10 \mathrm{~s}\right.$ at $1 \mu \mathrm{M}$, assuming bKL 338 association kinetics are similar to $\mathrm{KL}^{60}$, which has a rate constant of $10^{5} / \mathrm{M} / \mathrm{s}$ ). This separation of timescales 339 suggests that the assemblies should be able to form cotranscriptionally ${ }^{22,44}$ while tiles are being enzymatically 340 synthesized at a constant temperature of $37^{\circ} \mathrm{C}$.

341 We tested the cotranscriptional assembly of two representative Z-tiles, LZB13 and RZB14/25 (recall that, 342 when annealed, LZB13 predominantly forms linear twisted ladders, and RZB14/25 predominantly forms 343 annuli). Supplementary Fig. 15 shows AFM images of diluted cotranscriptional mixtures for both tiles, which 344 confirm that cotranscriptional assembly produces distributions of structures qualitatively similar to those formed 345 with annealing. The successful isothermal cotranscriptional assembly of these tiles encouraged us to explore 346 their expression and folding in the cells.

347 To generate RNA tiles in bacterial cells, we constructed a gene encoding the desired RNA tile, added an lpp 348 promoter and $\mathrm{rrnC}$ terminator, and inserted the construct between the SphI and BamHI restriction sites of the 349 vector pUC19 (Fig. 6a). Design of the tile involves addressing challenges not present for in vitro transcription. 350 To enhance the in vivo RNA stability and to demonstrate the tile's correct folding, we combined a basic Z-tile 351 (similar to LZB13) with two other RNA motifs (a tRNA scaffold ${ }^{61,62}$ and a Spinach aptamer ${ }^{63}$ ) to form a 352 chimera that we termed TSSP (a Z-tile within a tRNA scaffold with a Spinach aptamer) as shown in Fig. $6 \mathrm{~b}$ and 353 c. The unique fold of tRNA scaffold enables that the expressed RNA construct can be precisely processed by 354 cellular enzymes and escape cellular RNases, leading to better stability and cellular accumulation. Spinach 355 aptamer $^{63}$, which folds into an elongated conformation ${ }^{64}$ and fluoresces only if it binds its fluorophore 356 3,5-difluoro-4-hydroxybenzylidene imidazolinone (DFHBI), was fused to the anticodon loop of the tRNA ${ }^{65}$ at 357 its one end and connected to the strut of the Z-tile via a well-structured $3 \mathrm{WJ}^{66}$ from the phi29 prohead RNA at 358 its other end.

359 After transformation with TSSP expression vector and treatment with DFHBI, E. coli cells became highly 360 fluorescent (Fig. 6d). Because the Spinach aptamer sequence bookends both the 5' and 3' ends of the Z-tile 361 domain, fluorescence served as an indicator of the complete synthesis of both Spinach and the Z-tile domain. 362 Moreover, it indicated that Spinach could fold correctly in vivo when attached to a Z-tile domain. Further 363 experiments confirmed the full-length synthesis of TSSP tile and its competence for ladder assembly. Total 364 cellular RNA was extracted and analyzed by dPAGE; a sharp band corresponding to full-length TSSP (254-nt 365 long) was observed only for the cells transformed with the TSSP vector (Fig. 6e). Gel-purified TSSP RNA was 366 then annealed in vitro and imaged by AFM (Fig. 6f and Supplementary Fig. 43) and cryo-EM (Fig. 6g and 367 Supplementary Fig. 44). As expected, long filaments were observed by both techniques. Therefore, at least in 368 the case of in vitro thermal annealing, the insertion of the Spinach/tRNA fusion does not disturb the formation 369 of ladders. Given the above lines of evidence, the correct in vivo folding of TSSP tile and in vivo formation of 370 ladders are very likely, but definitive proof will require either a gentle, nondenaturing extraction of the ladders, 371 or high resolution cryo-EM tomography of thin bacterial cells. Nonetheless, the tRNA scaffold overexpression 372 373

374 bKL in RNA origami. A recently reported architecture for cotranscriptionally foldable RNA structures - RNA 375 origami-derives its generality from the repeated use of intramolecular $180^{\circ} \mathrm{KLs}$ and antiparallel four-way 376 junctions to organize dsRNA helices into large parallel arrays ${ }^{22}$. The paranemic cohesion provided by the 377 intramolecular KLs enables large RNA origami to be crafted from a single long strand. A major goal of RNA 378 nanoarchitectures is to eventually scaffold patterns of diverse proteins and small molecules via RNA 379 protein-binding motifs or aptamers ${ }^{67}$. Yet the first version of RNA origami left unclear how functional RNA 
380 motifs could be introduced at arbitrary positions within a structure. Grafting of $3 \mathrm{WJ}$ into helices provides one 381 possibility for incorporating functional RNAs, but the presence of crucial intramolecular KLs made this 382 approach impossible for a large number of positions and desired orientations. The bKL motif provides a perfect 383 solution to this problem. By introducing a geometrically well-defined branch exactly at the point of paranemic 384 cohesion, the bKL enables the insertion of a functional motif while maintaining the structural integrity of an 385 RNA origami helix. Figure 7a demonstrates this principle in the case of the simplest RNA origami structure, a 386 two-helix tile (DAE-bKL) designed to associate into $2 \mathrm{D}$ hexagonal lattices (Fig. $7 \mathrm{~b}$ ) via $120^{\circ} \mathrm{KL}$ motifs. 387 Replacement of a conventional KL with a bKL allows the precise positioning and orientation of a 388 protein-binding aptamer ${ }^{68}$ (Family 6 or F6, for the MS2 viral coat protein) without compromising the rigidity of 389 the tile or its assembly into a 2D lattice. High resolution AFM (Fig. 7c and Supplementary Fig. 45) confirms the 390 designed bKL-mediated orientation of F6 aptamers, with three copies pointing inward into each hexagonal 391 cavity.

392

393 DNA version of the bKL motif. Motifs for paranemic cohesion in DNA structures are less developed than 394 those for RNA and the majority of paranemically defined DNA structures depend on the relatively large and 395 complex PX motif, which joins two helices side-by-side ${ }^{45}$. Thus, it is of great interest to expand the vocabulary 396 of DNA paranemic motifs. Direct adaptation of HIV-1 type kissing loops (with its 2A-6N-1 A sequence motif) to 397 DNA was previously reported to result in paranemic cohesion, but with a "peculiar" configuration ${ }^{69}$, whose 398 idiosyncratic and noncanonical base pairings fail to lend themselves to generalization for the rational 399 engineering of nanostructures. By considering the differences between A-form RNA and B-form DNA, we have 400 been able to adapt the bKL motif from RNA to DNA with only minor modifications (Fig. 7d). Just as the case of 401 RNA bKL, we fused an extra stem to one hairpin loop to create a branch in the DNA bKL. Paranemic cohesion 402 was again provided by Watson-Crick base-pairs between a 6-nt bulge and a 6-nt region of a 9-nt hairpin loop. 403 However, for the DNA bKL, the remaining three unpaired nucleotides (dAs) of the loop were all positioned on 404 the 5' side of the binding region: the major groove of B-DNA (22 A) is wider than that of A-RNA (16 $\AA$ ), so we 405 add a linker containing three dAs to return the strand from the kissing region to the stem across the major 406 groove. We assumed coaxial stacking and modeled the resultant 6-bp kissing helix as contributing the same 407 twist as a standard 6-bp B-form DNA helix. Based on this DNA bKL, a one-stranded C-tile containing two 408 15-bp beams (dLCB15) was designed (Fig. 7e) and assembled into a ladder having no apparent twist when 409 imaged via AFM (Fig. 7f and Supplementary Fig. 46). The fact that two orthogonal and well-behaved DNA 410 bKL were so easily designed bodes well for the generality of this motif, and suggests that DNA KLs would also 411 work given a suitable exploration of linker lengths and sequences.

412

\section{Conclusions}

414

415

416

417

Our present work has introduced the bKL motif, which has been shown as a very desirable motif for RNA nanotechnology due to both its branched geometry and paranemic characteristic. Though artificially designed via the rational process of motif fusion, this bKL motif is essentially close to a number of different naturally occurring bulge-loop kissing interactions (which are also termed as bKLs here) in biological RNAs, including the group II intron ribozyme ${ }^{70}$ (an intramolecular 7-bp bKL is formed between the loop of stem IB and the bulge of stem ID2, i.e. the $\alpha-\alpha$ ' interaction), the RNase P RNA ${ }^{71,72}$ (the P6 in the archaeal and A-type bacterial RNase P RNA is formed by the intramolecular bKL interaction), and the $\Phi 29$ pRNA $^{73}$ (an intermolecular 4-bp $\mathrm{bKL}$ is formed between the loop $\mathrm{L}_{\mathrm{D}}$ and the bulge $\mathrm{L}_{\mathrm{CE}}$ ). Further, in vitro selection of aptamers targeting the hairpin loops in the untranslated domains of HCV mRNA has yielded new bulge motifs that also form bKL-type interactions with their target hairpin loops ${ }^{74,75}$. Therefore, it seems that our explorations of the basic bKL motif have barely scratched the surface of what is possible and what must be understood to best use bKLs in the engineering of nucleic acid-based nanostructures. For instance, both the number and position of the unpaired nucleotides within the bulge and the loop will likely have a profound impact on the flexibility, bending, and interhelical angle of the bKL motif. The number and sequence of base-pairs within the kissing helix, besides similarly affecting flexibility and geometry, could further dominate the thermodynamics $\left(K_{\mathrm{D}}\right)$ and kinetics $\left(k_{\mathrm{a}}\right.$ and $k_{\mathrm{d}}$ ) of the bKL interactions, which will be subject of our future research. Moreover, physicochemical 
431 properties (such as the in vivo stability) of the bKL-based nanostructures should also to be systematically 432 investigated for the practical biomedical applications as shown recently for similar RNA KL-based fibers ${ }^{44}$.

433 With respect to the existing practices in DNA and RNA nanotechnology, we expect that the bKL motif will 434 have a number of implications at the architectural level. Above, we demonstrated bKL as an avenue for 435 introducing a branch to augment RNA origami tiles with an aptamer. More generally, the bKL motif will 436 provide a versatile alternative for elaborating RNA and DNA structures into the third dimension, especially in 437 large one-stranded RNA and DNA origami ${ }^{31}$. For such structures, the introduction of bKL will greatly increase 438 the number of available strand paths (i.e. the routing of the single strand through the structure). Currently, 439 branches in RNA and DNA origami are mediated by multi-armed junctions, and these motifs somewhat restrict 440 the complexity of strand paths that can be designed without introducing topological problems and concomitant 441 kinetic traps. Appropriate deployment of bKLs in the design may thus help these structures achieve more local 442 folding and higher yields.

443 Further, the programming of curvature, twist, and flexibility in both one-stranded origami and 444 multi-stranded tiles presents further opportunities for bKL to advance nucleic acid-based architectures. Current 445 approaches to curvature and twist in $2 \mathrm{D}^{76}$ and $3 \mathrm{D}^{77,78}$ DNA origami rely on changing the number of base pairs 446 between crossovers to deviate from the periodicity of the ideal B-form helix $\left(10.4 \pm 0.1\right.$ bps per helical turn $\left.^{79}\right)$ : 447 the desired curvature and twist are generated via tuning the release and balancing of structural strain. The 448 replacement of crossovers by struts, though requiring some further recalibration, would enable the programming 449 of equally arbitrary curves in bKL-based origami. Alternatively, we envision that a library of bKL-based tiles of 450 known curvature and twist could be built, so that arbitrary space curves could be designed and assembled 451 quickly and easily, in a modular fashion. Current approaches to controlling flexibility of a nucleic acid structure 452 involve choosing the number and pattern of parallel helices (analogous to rails) which define the structure's 453 cross-section $^{80}$. However, the use of crossovers to hold such helices together means that the number of helices 454 and spacing between helices cannot be independently changed. Multi-railed bKL-based structures, like those we 455 have demonstrated in Fig. 4, will provide an opportunity to simultaneously control the number of parallel 456 helices in a structure, and the bending moment of inertia (and hence the stiffness), simply via changing the 457 struts' lengths.

458 Finally, we return to the idea of the dynamic regulation of homooligomeric structures, which we have not 459 realized in the current work. The dynamic behaviors of the canonical examples, such as microtubules ${ }^{5}$ and actin 460 filaments $^{6}$, are controlled by a number of mechanisms, most of which are fundamentally driven by coupling to 461 nucleotide hydrolysis - a reaction we do not yet know how to convert into a conformational change or alteration 462 of affinity for RNA tiles. Nonetheless, dynamic control over homooligomeric RNA structures may be effected 463 by other methods: a wide range of protein and small molecule-induced conformational changes, as well as 464 catalytic functions, have been seen in both natural and in vitro selected/evolved RNA molecules ${ }^{81}$. For example, 465 simple addition of L7ae to flexible RNA loops with three copies of an appropriate K-turn motif converts the 466 loops into equilateral triangles ${ }^{82}$. Admittedly, the integration of functional and structural RNA motifs to create 467 homooligomeric systems that switch robustly and controllably between two or more rigid and well-defined 468 geometries will require considerable ingenuity. Probably, the bulges and loops of bKL-based tiles, besides 469 providing cohesion, could also be engineered as small molecule aptamers to modulate the inter-tile affinity. 470 Further, since small perturbations to beams and struts could yield large overall changes in the resulting 471 nanostructures, addition of protein binding sites into these helical segments may readily create large 472 conformational changes when bound to relevant proteins. Given these possibilities, bKL-based tiles seem to be 473 ideal starting point from which to engineer dynamic nucleic acid-based homooligomeric systems.

\section{Methods}

RNA preparation. Sequences were designed via sequence symmetry minimization ${ }^{83}$ using CANADA ${ }^{84}$. To ensure correct folding, we verified that the desired secondary structure for each design was predicted to be the minimum free energy structure by Mfold ${ }^{85}$. RNA sequences and predicted secondary structures for various bKL-based C- and Z-tiles are shown in Supplementary Figs. 2 to 5; secondary structures were prepared with the assistance of VARNA ${ }^{86}$. Corresponding DNA template sequences for these RNA tiles are listed in Supplementary Table 1. Sequences used to prepare DAE-bKL tile are listed in Supplementary Table 2. For all tiles except DAE-bKL, the first two nucleotides of reverse PCR primers were modified with a 2'-OMe group to reduce transcriptional heterogeneity at the $3^{\prime}$ end of transcripts ${ }^{87}$. It should be noted that though we have constructed various RNA assemblies in this work, most of these structures shared essentially the same workflow including RNA preparation and purification, assembly, and structure 
characterizations. All RNA molecules were synthesized by in vitro transcription using the HiScribe ${ }^{\mathrm{TM}} \mathrm{T} 7$ High Yield RNA Synthesis Kit from the New England Biolabs (NEB). Corresponding DNA templates were generated by the PCR amplification of the gBlocks ${ }^{\circledR}$ gene fragments from the Integrated DNA Technologies (IDT) using the Q $5^{\circledR}$ Hot Start High-Fidelity DNA Polymerase (NEB). Except where cotranscriptionally folded, all RNAs were purified by denaturing PAGE (dPAGE), ethanol precipitated and suspended in pure water.

RNA nanostructure assembly. Before assembly, RNAs were denatured at $90^{\circ} \mathrm{C}$ for $1 \mathrm{~min}$ and snap-cooled on ice. For the assembly, unless stated otherwise, RNAs were diluted to $600 \mathrm{nM}$ in $1 \times$ TAE-Mg buffer $(11 \mathrm{mM} \mathrm{MgCl}, 40 \mathrm{mM}$ Tris, $20 \mathrm{mM}$ acetic acid, $1 \mathrm{mM}$ EDTA, $\mathrm{pH} 8.0$ ) and annealed from $70^{\circ} \mathrm{C}$ to $4^{\circ} \mathrm{C}$ in three stages: $70^{\circ} \mathrm{C}$ to $50^{\circ} \mathrm{C}$ over $6 \min , 50^{\circ} \mathrm{C}$ to $37{ }^{\circ} \mathrm{C}$ over 20 min, $37^{\circ} \mathrm{C}$ to $4{ }^{\circ} \mathrm{C}$ over $2 \mathrm{hr}$. To get a purer fraction of single-layered annuli assembled by RZB14/36 tiles, the assembly mixture was filtered with Spin- $\mathrm{X}^{\circledR}$ centrifuge tube filters $\left(0.22 \mu \mathrm{m}\right.$ pore size, Corning $\left.{ }^{\circledR} \mathrm{Costar}^{\circledR}\right)$ under $1000 \times \mathrm{g}$ for 2 minutes at $4{ }^{\circ} \mathrm{C}$. For CZB12b11 nanocages, buffer composition was optimized mixing varying ratios of $10 \times \mathrm{TAE}-\mathrm{Mg}$ buffer and $1 \mathrm{M} \mathrm{NaCl}$, and yield was maximized at $0.3 \mathrm{mM}$ $\mathrm{Mg}^{2+}$ and $100 \mathrm{mM} \mathrm{Na}^{+}$.

Cellular production and fluorescence characterization of of TSSP. The gene for expressing TSSP (sequences are in Supplementary Table 3) was cloned into the BamH I + Sph I restriction sites of vector pUC19 and transformed into DH5 $\alpha$ competent cells. Cells were grown overnight in fresh $\mathrm{LB}$ medium containing $50 \mu \mathrm{g} / \mathrm{mL}$ ampicillin at $37^{\circ} \mathrm{C}$ to an $\mathrm{OD}_{600 \mathrm{~nm}}$ of $\sim 0.6-0.8$. Total RNA was prepared by phenol/chloroform/isoamyl alcohol extraction and ethanol precipitation. For in vitro assembly, the target RNA TSSP was purified by dPAGE. Fluorescence characterization was performed on intact bacterial cells in the presence of $20 \mu \mathrm{M}$ 3,5-difluoro-4-hydroxybenzylidene imidazolinone (DFHBI, synthesized according to a published method ${ }^{63}$ ). Fluorescence spectroscopy was performed by a Synergy H4 Hybrid Multi-Mode Microplate Reader (BioTek) and fluorescence photography performed in the darkroom of an EC3 bioimaging system (UVP).

DNA preparation. Tile dLCB15 was synthesized via splinted DNA ligation from two precursor strands, using hybridization with a splint strand (sequences in Supplementary Table 4) using T4 DNA ligase (NEB). After ligation, the full-length tile strand was purified by dPAGE, and refolded via annealing similar to that for RNA tiles.

AFM imaging. Post-annealing, reaction mixtures were diluted 10 times with $1 \times \mathrm{TAE}-\mathrm{Mg}$ buffer. $5 \mu \mathrm{L}$ of the diluted sample was deposited onto a freshly cleaved mica surface (Ted Pella) and left to adsorb for 1 min before being dried with compressed air. The mica was then rinsed with $20 \mu \mathrm{L}$ of $2 \mathrm{mM} \mathrm{Mg}(\mathrm{OAc})_{2}$ and dried with compressed air. AFM images were acquired on a Multimode 8 AFM in the "ScanAsyst ${ }^{\circledR}$ in Air" mode using ScanAsyst-air tips (Bruker) and processed with Gwyddion.

Cryo-EM imaging and single-particle reconstruction. For all structures except the tetrameric nanocages, the post-anneal reaction mixture containing $600 \mathrm{nM}$ of RNA tiles was directly used for grid preparation. For the nanocages, the post-anneal reaction mixture was concentrated 10 times with an Amicon Ultra centrifugal filter (MWCO $30 \mathrm{kDa}$ ) before grid preparation. In general, $3 \mu \mathrm{L}$ of solution was applied onto a glow-discharged C-flat ${ }^{\mathrm{TM}}$ holey carbon grid (CF-1.2/1.3-4C), blotted for 5.5 s and immediately flash frozen by liquid nitrogen-cooled liquid ethane with a Cryoplunge 3 System (GATAN). Images were collected on a JEOL 3200FS TEM operated at $300 \mathrm{kV}$ equipped with a K2 Summit direct electron counting camera (GATAN) under low-dose mode. For the structures without single-particle reconstruction, images were recorded at $12000 \times$ or $25000 \times$ microscope magnification with the defocus ranging from about $-3.0 \mu \mathrm{m}$ to $-5.0 \mu \mathrm{m}$. For single-particle reconstruction of the nanocages, images were recorded at $25000 \times$ microscope magnification with the defocus ranging from about $-1.0 \mu \mathrm{m}$ to $-4.0 \mu \mathrm{m}$.

Single-particle reconstruction was performed using EMAN2 ${ }^{88} .598$ particles were used to generate reference-free class averages, which were used to build the initial model. Refinement of the model was conducted with the full set of 1440 particles. Resolution of the resulting structural density map was estimated to be $18.1 \AA$ using the gold-standard FSC $=0.143$ criterion, without applying any mask. The reconstructed model was visualized by UCSF Chimera ${ }^{89}$.

Data availability. The data supporting the findings of this study are principally within the figures and the associated Supplementary Information. Additional data are available from the authors upon request. 


\section{References}

Labeit, S. \& Kolmerer, B. Titins: Giant Proteins in Charge of Muscle Ultrastructure and Elasticity. Science 1995, 270(5234): 293-296.

Goodsell, D. S. \& Olson, A. J. Structural symmetry and protein function. Annu. Rev. Biophys. Biomol. Struct. 2000, 29: 105-153.

Ali, M. H. \& Imperiali, B. Protein oligomerization: how and why. Bioorg Med Chem 2005, 13(17): 5013-5020.

Pieters, B. J., van Eldijk, M. B., Nolte, R. J. \& Mecinovic, J. Natural supramolecular protein assemblies. Chem Soc Rev 2016, 45(1): 24-39.

Desai, A. \& Mitchison, T. J. Microtubule polymerization dynamics. Annu Rev Cell Dev Biol 1997, 13: 83-117.

Bugyi, B. \& Carlier, M. F. Control of actin filament treadmilling in cell motility. Annu Rev Biophys 2010, 39: 449-470.

Mitchison, T. J. \& Cramer, L. P. Actin-Based Cell Motility and Cell Locomotion. Cell 1996, 84(3): 371-379.

Seeman, N. C. Nanomaterials based on DNA. Annu. Rev. Biochem. 2010, 79: 65-87.

Zhang, F., Nangreave, J., Liu, Y. \& Yan, H. Structural DNA nanotechnology: state of the art and future perspective. J. Am. Chem. Soc. 2014, 136(32): 11198-11211.

Seeman, N. C. Structural DNA Nanotechnology. Cambridge University Press, 2016.

Guo, P. The emerging field of RNA nanotechnology. Nature Nanotechnol. 2010, 5(12): 833-842.

Paillart, J. C., Marquet, R., Skripkin, E., Ehresmann, C. \& Ehresmann, B. Dimerization of retroviral genomic RNAs: structural and functional implications. Biochimie 1996, 78(7): 639-653.

Hill, A. C., Bartley, L. E. \& Schroeder, S. J. Prohead RNA: a noncoding viral RNA of novel structure and function. Wiley Interdiscip Rev RNA 2016, 7(4): 428-437.

Winfree, E., Liu, F., Wenzler, L. A. \& Seeman, N. C. Design and self-assembly of two-dimensional DNA crystals. Nature 1998, 394(6693): 539-544.

Rothemund, P. W. et al. Design and characterization of programmable DNA nanotubes. J. Am. Chem. Soc. 2004, 126(50): 16344-16352.

Stewart, J. M., Subramanian, H. K. K. \& Franco, E. Self-assembly of multi-stranded RNA motifs into lattices and tubular structures. Nucleic Acids Res. 2017, 45(9): 5449-5457.

Liu, H., Chen, Y., He, Y., Ribbe, A. E. \& Mao, C. Approaching the limit: can one DNA oligonucleotide assemble into large nanostructures? Angew. Chem. Int. Ed. 2006, 45(12): 1942-1945.

Tian, C. et al. Approaching the limit: can one DNA strand assemble into defined nanostructures? Langmuir 2014, 30(20): 5859-5862.

Li, M., Zuo, H., Yu, J., Zhao, X. \& Mao, C. One DNA strand homo-polymerizes into defined nanostructures. Nanoscale 2017, 9(30): 10601-10605.

Horiya, S. et al. RNA LEGO: magnesium-dependent formation of specific RNA assemblies through kissing interactions. Chemistry \& Biology 2003, 10(7): 645-654.

Nasalean, L., Baudrey, S., Leontis, N. B. \& Jaeger, L. Controlling RNA self-assembly to form filaments. Nucleic Acids Res. 2006, 34(5): 1381-1392.

Geary, C., Rothemund, P. W. \& Andersen, E. S. RNA nanostructures. A single-stranded architecture for cotranscriptional folding of RNA nanostructures. Science 2014, 345(6198): 799-804.

Praetorius, F. et al. Biotechnological mass production of DNA origami. Nature 2017, 552(7683): 84-87.

Heiat, M., Ranjbar, R., Latifi, A. M., Rasaee, M. J. \& Farnoosh, G. Essential strategies to optimize asymmetric PCR conditions as a reliable method to generate large amount of ssDNA aptamers. Biotechnol. Appl. Biochem. 2017, 64(4): 541-548.

Veneziano, R. et al. In vitro synthesis of gene-length single-stranded DNA. Sci. Rep. 2018, 8(1): 6548.

Jasinski, D., Haque, F., Binzel, D. W. \& Guo, P. Advancement of the Emerging Field of RNA Nanotechnology. ACS nano 2017, 11(2): 1142-1164.

Wang, H., Di Gate, R. J. \& Seeman, N. C. An RNA topoisomerase. Proc. Natl. Acad. Sci. USA 1996, 93(18): 9477-9482.

Liu, D. et al. Synthesizing topological structures containing RNA. Nature Commun. 2017, 8: 14936.

Afonin, K. A. et al. In vitro assembly of cubic RNA-based scaffolds designed in silico. Nat Nanotechnol 2010, 5(9): 676-682. Endo, M., Takeuchi, Y., Emura, T., Hidaka, K. \& Sugiyama, H. Preparation of chemically modified RNA origami nanostructures. Chem. Eur. J. 2014, 20(47): 15330-15333.

Han, D. et al. Single-stranded DNA and RNA origami. Science 2017, 358(6369): eaao2648.

Severcan, I. et al. A polyhedron made of tRNAs. Nat Chem 2010, 2(9): 772-779.

Hao, C. et al. Construction of RNA nanocages by re-engineering the packaging RNA of Phi29 bacteriophage. Nat Commun 2014, 5: 3890.

Shu, D., Moll, W. D., Deng, Z., Mao, C. \& Guo, P. Bottom-up Assembly of RNA Arrays and Superstructures as Potential Parts in Nanotechnology. Nano Lett 2004, 4(9): 1717-1723.

35. Dibrov, S. M., McLean, J., Parsons, J. \& Hermann, T. Self-assembling RNA square. Proc. Natl. Acad. Sci. USA 2011, 108(16): 
Boerneke, M. A., Dibrov, S. M. \& Hermann, T. Crystal-Structure-Guided Design of Self-Assembling RNA Nanotriangles. Angew. Chem. Int. Ed. 2016, 55(12): 4097-4100.

Khisamutdinov, E. F. et al. Enhancing immunomodulation on innate immunity by shape transition among RNA triangle, square and pentagon nanovehicles. Nucleic Acids Res. 2014, 42(15): 9996-10004. Structural Modules. Nano Lett 2017, 17(11): 7095-7101. 878-887. Adv. Funct. Mater. 2018, 28(48).

Zhang, X., Yan, H., Shen, Z. \& Seeman, N. C. Paranemic Cohesion of Topologically-Closed DNA Molecules. J. Am. Chem. Soc. 2002, 124(44): 12940-12941.

Afonin, K. A., Cieply, D. J. \& Leontis, N. B. Specific RNA self-assembly with minimal paranemic motifs. J. Am. Chem. Soc. 2008, 130(1): 93-102.

Ennifar, E., Walter, P., Ehresmann, B., Ehresmann, C. \& Dumas, P. Crystal structures of coaxially stacked kissing complexes of the HIV-1 RNA dimerization initiation site. Nature Struct. Biol. 2001, 8(12): 1064-1068.

Hamada, S. \& Murata, S. Substrate-assisted assembly of interconnected single-duplex DNA nanostructures. Angew. Chem. Int. Ed. 2009, 48(37): 6820-6823.

Fiore, J. L. \& Nesbitt, D. J. An RNA folding motif: GNRA tetraloop-receptor interactions. Q. Rev. Biophys. 2013, 46(3): 223-264.

Kieken, F., Paquet, F., Brule, F., Paoletti, J. \& Lancelot, G. A new NMR solution structure of the SL1 HIV-1Lai loop-loop dimer. Nucleic Acids Res. 2006, 34(1): 343-352.

Yan, H., Park, S. H., Finkelstein, G., Reif, J. H. \& LaBean, T. H. DNA-templated self-assembly of protein arrays and highly conductive nanowires. Science 2003, 301(5641): 1882-1884.

Woo, S. \& Rothemund, P. W. Programmable molecular recognition based on the geometry of DNA nanostructures. Nat Chem 2011, 3(8): 620-627.

Gerling, T., Wagenbauer, K. F., Neuner, A. M. \& Dietz, H. Dynamic DNA devices and assemblies formed by shape-complementary, non-base pairing 3D components. Science 2015, 347(6229): 1446-1452.

Bates, A. D., Noy, A., Piperakis, M. M., Harris, S. A. \& Maxwell, A. Small DNA circles as probes of DNA topology. Biochem Soc Trans 2013, 41(2): 565-570.

Dibrov, S. M., Johnston-Cox, H., Weng, Y. H. \& Hermann, T. Functional architecture of HCV IRES domain II stabilized by divalent metal ions in the crystal and in solution. Angew. Chem. Int. Ed. 2007, 46(1-2): 226-229.

Yu, J., Liu, Z., Jiang, W., Wang, G. \& Mao, C. De novo design of an RNA tile that self-assembles into a homo-octameric nanoprism. Nat Commun 2015, 6: 5724.

Woodson, S. A. Metal ions and RNA folding: a highly charged topic with a dynamic future. Curr Opin Chem Biol 2005, 9(2): 104-109.

Heilman-Miller, S. L. Effect of transcription on folding of the Tetrahymena ribozyme. Rna 2003, 9(6): 722-733.

Cruz, J. A. \& Westhof, E. The dynamic landscapes of RNA architecture. Cell 2009, 136(4): 604-609.

Rist, M. \& Marino, J. Association of an RNA kissing complex analyzed using 2-aminopurine fluorescence. Nucleic Acids Res. 2001, 29(11): 2401-2408.

Ponchon, L. \& Dardel, F. Recombinant RNA technology: the tRNA scaffold. Nat Methods 2007, 4(7): 571-576.

Ponchon, L., Beauvais, G., Nonin-Lecomte, S. \& Dardel, F. A generic protocol for the expression and purification of recombinant RNA in Escherichia coli using a tRNA scaffold. Nat Protoc 2009, 4(6): 947-959.

Paige, J. S., Wu, K. Y. \& Jaffrey, S. R. RNA mimics of green fluorescent protein. Science 2011, 333(6042): 642-646.

Huang, H. et al. A G-quadruplex-containing RNA activates fluorescence in a GFP-like fluorophore. Nat Chem Biol 2014, 10(8): 686-691.

BÉNas, P. et al. The crystal structure of HIV reverse-transcription primer tRNA(Lys,3) shows a canonical anticodon loop. Rna 2000, 6(10): 1347-1355.

Zhang, H. et al. Crystal structure of 3WJ core revealing divalent ion-promoted thermostability and assembly of the Phi29 hexameric motor pRNA. RNA 2013, 19(9): 1226-1237.

67. Delebecque, C. J., Lindner, A. B., Silver, P. A. \& Aldaye, F. A. Organization of intracellular reactions with rationally designed RNA assemblies. Science 2011, 333(6041): 470-474. 

1998, 5(2): 133-139.

646 69. Barbault, F., Huynh-Dinh, T., Paoletti, J. \& Lanceloti, G. A new peculiar DNA structure: NMR solution structure of a DNA kissing complex. J Biomol Struct Dyn 2002, 19(4): 649-658. Toor, N., Keating, K. S., Taylor, S. D. \& Pyle, A. M. Crystal structure of a self-spliced group II intron. Science 2008, 320(5872): 77-82. 333-341. bacterial ribonuclease P. Nature 2005, 437(7058): 584-587. RNA-RNA Recognition Motif Identified through in Vitro Selection against RNA Hairpins of the Hepatitis C Virus mRNAt. Biochemistry 2002, 41(18): 5883-5893.

Da Rocha Gomes, S., Dausse, E. \& Toulme, J. J. Determinants of apical loop-internal loop RNA-RNA interactions involving the HCV IRES. Biochem Biophys Res Commun 2004, 322(3): 820-826.

Zhang, F. et al. Complex wireframe DNA origami nanostructures with multi-arm junction vertices. Nature Nanotechnol. 2015, 10(9): 779-784. 725-730. 6700-6710.

Breaker, R. R. \& Joyce, G. F. The expanding view of RNA and DNA function. Chem. Biol. 2014, 21(9): 1059-1065.

83. Seeman, N. C. Nucleic acid junctions and lattices. J. Theor. Biol. 1982, 99(2): 237-247.

84. Feldkamp, U. CANADA: Designing Nucleic Acid Sequences for Nanobiotechnology Applications. J. Comput. Chem. 2010, 31(3): 660-663.

85. Zuker, M. Mfold web server for nucleic acid folding and hybridization prediction. Nucleic Acids Res. 2003, 31(13): $3406-3415$.

6. Darty, K., Denise, A. \& Ponty, Y. VARNA: Interactive drawing and editing of the RNA secondary structure. Bioinformatics 2009, 25(15): 1974-1975.

Acknowledgments D. L. acknowledges the HHMI International Student Research Fellowship. C.W.G. acknowledges a fellowship from the Carlsberg Research Foundation. This work was supported by NSF CAREER Award (DMR-1555361) to Y. W., NIH grant (R01GM102489) to J.A.P., ERC grant (683305) to E.S.A., and NSF grants (CCF-1317694 and CMMI-1636364) and ONR grant (N00014-16-1-2159) to P.W.K.R. Cryo-EM experiments were conducted with the Structural Biology Facility at Northwestern University and we thank Jonathan Remis for the assistance. We thank Nan-sheng Li for synthesizing DFHBI. terminus of RNAs transcribed by T7 RNA polymerase. RNA 1999, 5(9): 1268-1272.

Tang, G. et al. EMAN2: an extensible image processing suite for electron microscopy. J Struct Biol 2007, 157(1): 38-46. 25(13): 1605-1612.

\section{4}


Figures with captions.

699

a kissing loop (KL)

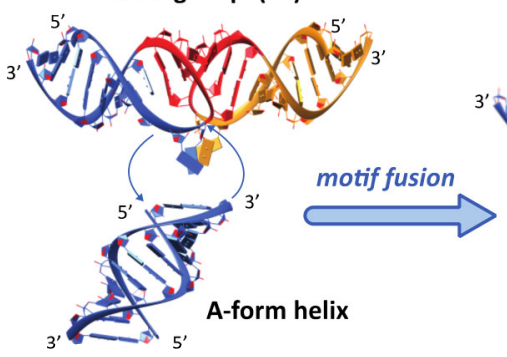

b
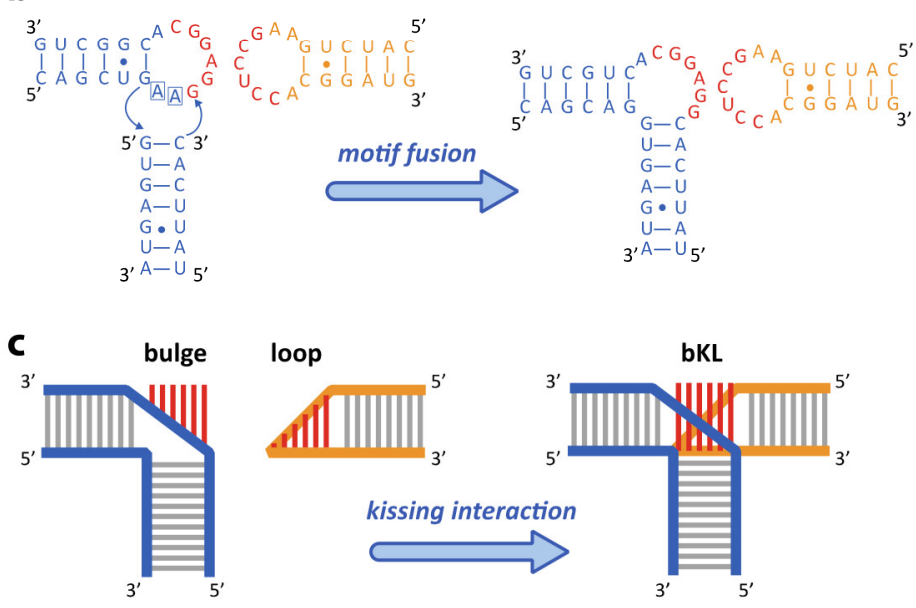
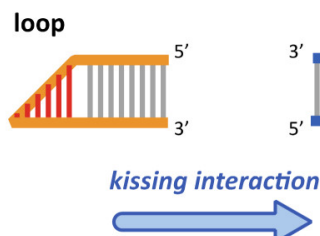

700

701

702

703

704

705

706

707

708

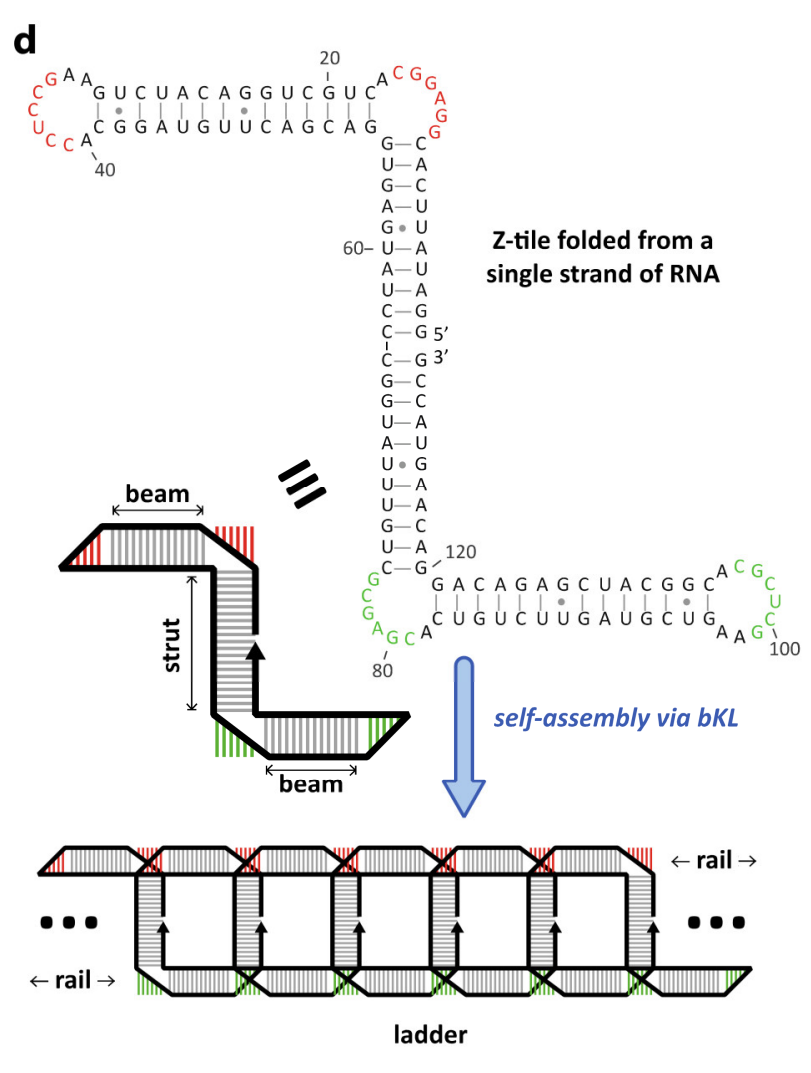

Figure 1 | The branched kissing loop (bKL) motif. a, b, Helical representation (a) and exemplar sequence details (b) of the process used to design a bKL from the fusion of a coaxially-stacked linear KL (top, adapted from that in the HIV DIS) with an additional A-form helix (bottom). The two hairpins of the KL bind via six Watson-Crick base-pairs between red-colored nucleotides in their loop regions; the remaining nucleotides of each hairpin (colored blue at left and orange at right) include based-paired stems, and three unpaired adenines. By replacing two of the unpaired adenines (boxed in b) in the left-hand hairpin with an A-form helix, the left-hand loop is converted into a bulge that can still base-pair with the loop of the right-hand hairpin. c, The formation of a bKL, colored to match $\mathbf{a}$ and $\mathbf{b}$. d, The use of bKL motifs to create a one-stranded Z-tile that self-assembles into a higher-order ladder. 

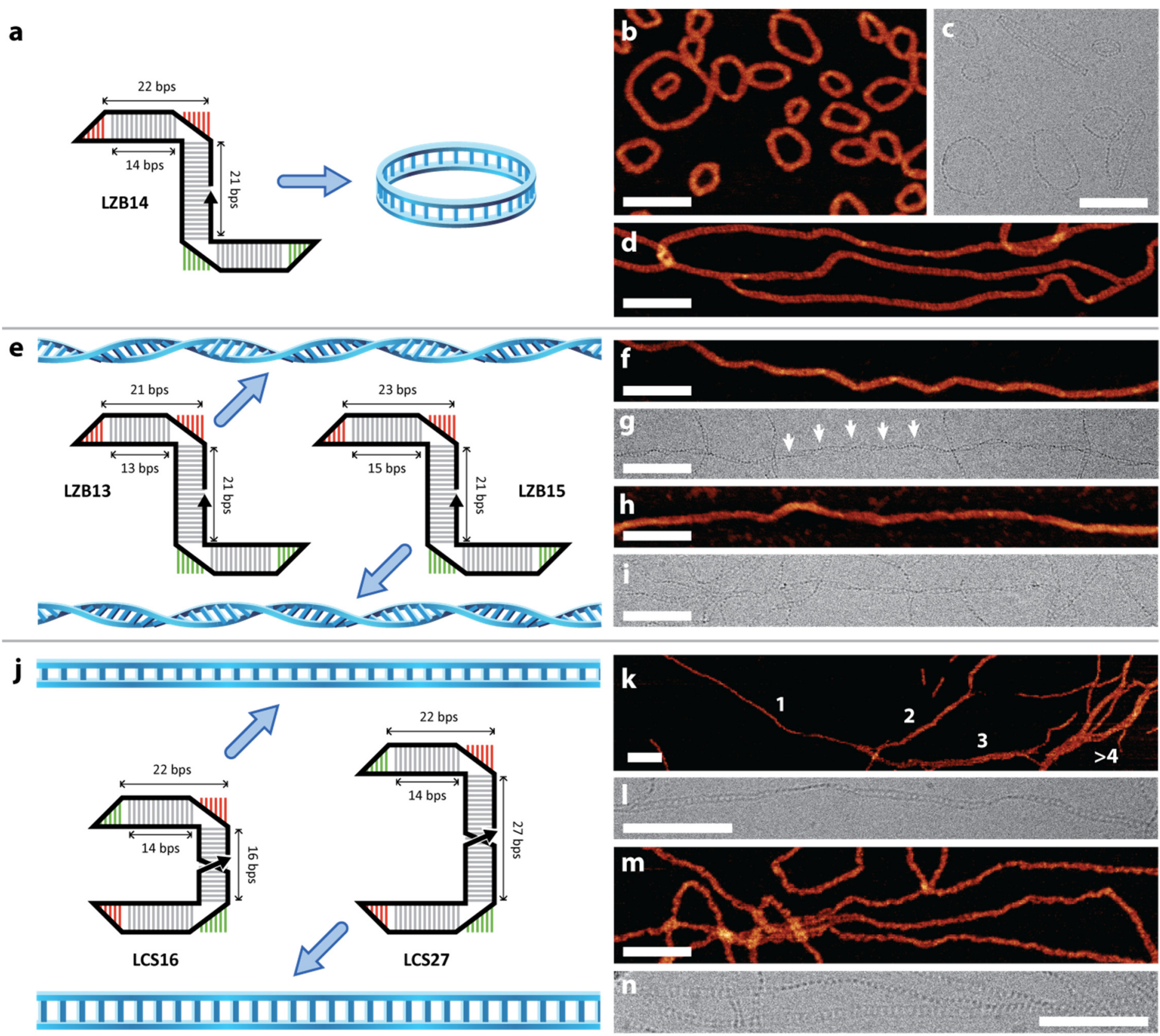

710 Figure 2 | Effects of beam and strut lengths on ladders assembled from RNA tiles having beams of equal length. Schematics are shown at left; corresponding AFM (color) and cryo-EM (gray) at right. a, To achieve an integral number (here two)of helical turns, and thereby minimize torsion, the Z-tile (LZB14) was designed with 14-bp beams. b-d, In the absence of torsion, Z-tile symmetry allowed out-of-plane curvature to accumulate, causing the formation of closed rings of mostly small (b,c) or occasionally large (d) diameters. e-i, By decreasing or increasing beam length by one base pair in the designs of LZB13 and LZB15, respectively (e), left$(\mathbf{f}, \mathbf{g})$ and right-handed $(\mathbf{h}, \mathbf{i})$ twisted ladders were produced. From cryo-EM images $(\mathbf{g}, \mathbf{i})$, we could directly measure the twist of the ladders (16-18 tiles per helical turn for the LZB13 ladder, and 17-21 tiles per helical turn for LZB15 ladder). The observed ladder twist was smaller than the maximum possible for the designs - the twist due to addition or removal of one base pair would accumulate to create a single turn of the ladder with roughly every 11 tiles. No fixed superhelical features (e.g. additional longer helical period) were observed. Therefore, the excess twist is likely to be relaxed via the intrinsic flexibility of the bKL motifs in the assembled ladders. Arrows in $\mathbf{g}$ indicate examples of nodes between half-turns which were used to measure the number of tiles per turn. $\mathbf{j}$-n, Changing the strut length of LZB14 from an even number to an odd number of half-turns results in the C-tiles LCS16 (three half turns, $16 \mathrm{bp}$ ) and LCS27 (five half turns, $27 \mathrm{bp}$ ) (j). These C-tiles created a screw axis symmetry that minimized curvature and torsion in corresponding ladders ( $\mathbf{k}, \mathbf{l}$ for LCS16; $\mathbf{m}, \mathbf{n}$ for LCS27). Wider structures resulting from stacking of ladders are evident in $\mathbf{k}$, probably due to AFM sample preparation, and numeric labels indicate the estimated number of stacked ladders in each filament. Scale bars: $100 \mathrm{~nm}$. The nomenclature of each tile in this work is explained in Supplementary Fig. 2. 

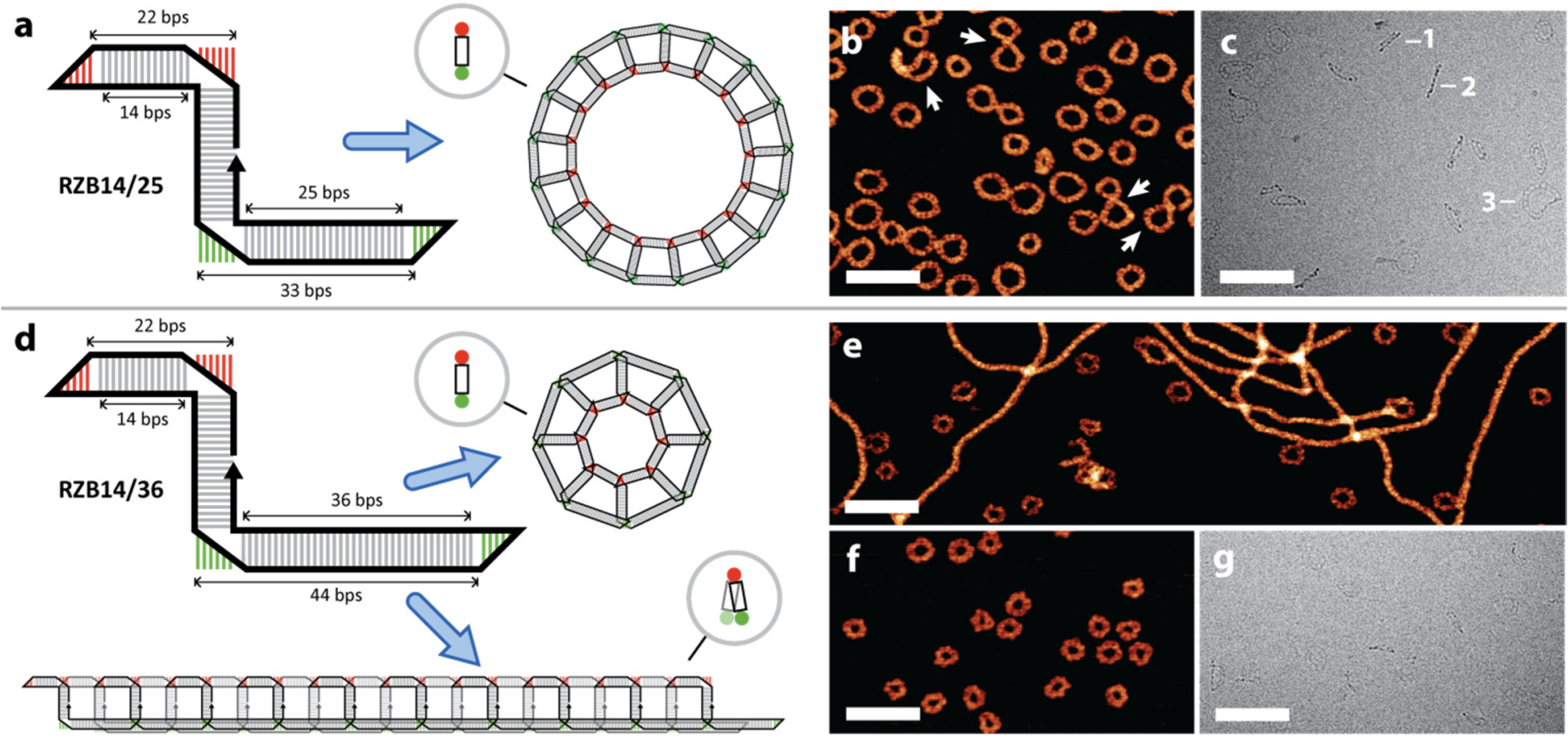

h
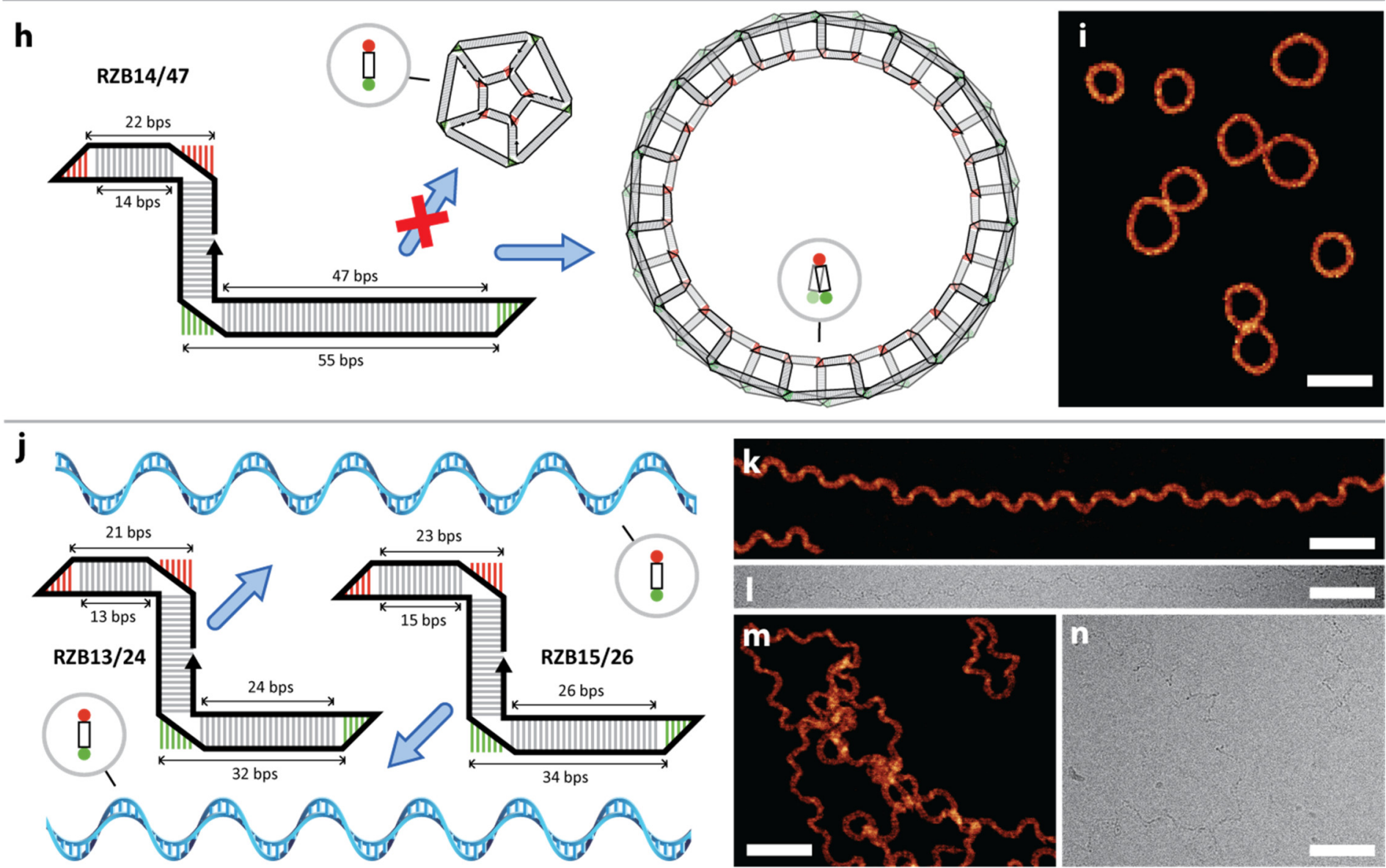

Figure 3 | Effect of beam and strut length in tiles having unequal beams lengths. a, RZB14/25, whose beams differ by one helical 730 turn, was predicted to form annuli of $47-50 \mathrm{~nm}$ diameter (containing 16 or 17 tiles). The cross-section is shown within a gray circle. $\mathbf{b}$, 731 c, Experimentally, 36-50 nm annuli and other relevant shapes are observed. White arrows in AFM (b) show figure of eights and other 732 forms, which are hypothesized to be larger nonplanar assemblies (containing up to 25 tiles), and collapse on the surface. In cryo-EM 733 (c), three different shapes were indicated: (1) frustum; (2) planar annulus; (3) saddle. d, RZB14/36, whose beams differ by two turns, 734 was predicted to form 31 or $35 \mathrm{~nm}$ annuli. e-g, Experimentally, both 26-38 nm annuli and micron long filaments of 735 higher-than-expected height were observed (e-g; filaments were removed by $0.22-\mu \mathrm{m}$ filter for $\mathbf{f}$ and $\mathbf{g}$ ). Long filaments were

736 interpreted to be three-railed structures with a triangular cross section, having regions of both double and single layers of RNA helix. 737 A model of the filament with two of the rails (green) on top of each other is shown at $\mathbf{d}$ (bottom). h, RZB14/47, whose beams differ by 
738 three turns, self-assembles into double-layered annulus structures because the formation of the single-layered annulus (5- or 6-tile, and

73925 or $29 \mathrm{~nm}$ in diameter) would require a curvature that is too large for the tile to accommodate. i, 55-86 nm annuli of

740 higher-than-expected height were observed, which corresponds to a circumference of 11 to 18 tiles (i.e. 22 to 36 tiles in total). This is

741 consistent with the model of a double-layered annulus, which is predicted by geometrical analysis to have a circumference of 16 or 17

742 tiles if planar (right of $\mathbf{h}$, also Supplementary Fig. 13 for more details). In each double-layered annulus, the single-layered inner rail

743 comprises the shorter beam of every tile, and either of the two outer rails comprises the longer beam of every other tile. $\mathbf{j}$, Torsion and

744 in-plane curvature combine to create helical ladders (termed as nano-ramens). RZB13/24 and RZB15/26 were designed by

745 performing base-pair deletion and insertion on RZB14/25 to have left- and right-handed torsion, respectively. In each such

746 nano-ramen, the two rails approximately trace out a pair of Bertrand curves (space curves which share normal lines; the strut provides

747 a common normal to both rails, assuming that the bKL are perfect T-shapes). k-n, $55 \mathrm{~nm}$ period nano-ramen (RZB13/24 in k,l) and 60

$748 \mathrm{~nm}$ period nano-ramen (RZB15/26 in m,n) were observed. Scale bars: $100 \mathrm{~nm}$. 



Figure 4 | Multi-railed ladders. a, LZB19/47 self-assembles into a three-railed ladder. Because the distances $2 S=54$ bp (along the central rail) and $L=55 \mathrm{bp}$ (along a peripheral rail) differ by a single base pair over a five-turn span, we expected a ladder twist with a period of 11 tiles or $169 \mathrm{~nm}$. Measurements of the distances between visible nodes (indicated by white arrows in cryo-EM) in the ladders gave a somewhat shorter period of $104 \mathrm{~nm} \pm 18 \mathrm{~nm}(N=10$ half periods) with roughly 7 tiles. b, LZB10/47 self-assembles into a four-railed ladder. While the difference between distances $3 S=54$ bp and $L=55$ bp predicts significant twist for RZB10/47 ladders, their three peripheral rails could not be easily differentiated in cryo-EM, making the estimation of twist impossible. c, A three-way junction (3WJ) is used to connect three half-C-tiles to form the claw-like tile LCS3WJ, which self-assembles into a three-railed ladder. d, A four-way junction (4WJ) connects two C-tiles to form LCS4WJ, which self-assembles into a four-railed ladder. Both structures assembled from LCS3WJ and LCS4WJ should be of minimal twist. Indeed, inspection of their respective cryo-EM images reveals that long tracts of the same pattern can be conserved over a long distance. Scale bars: $50 \mathrm{~nm}$. 
a
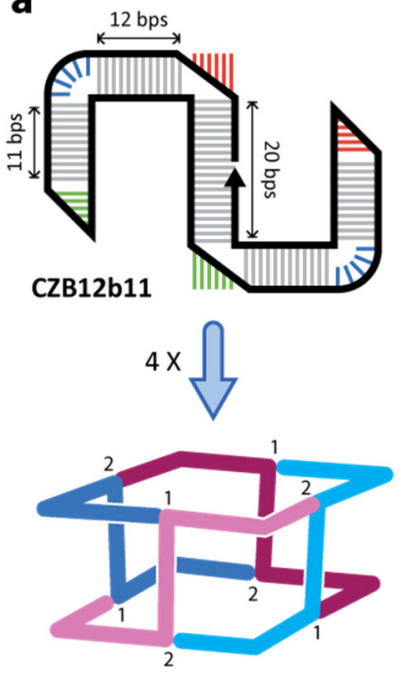

b
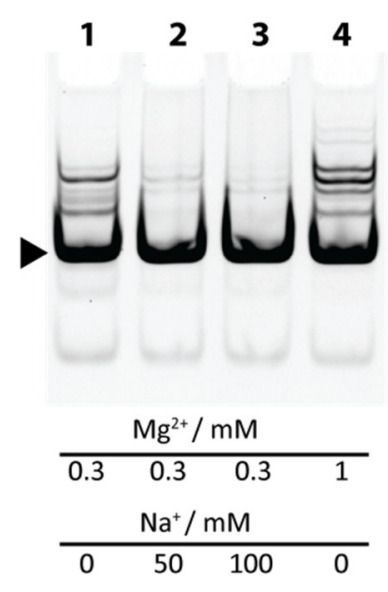



e

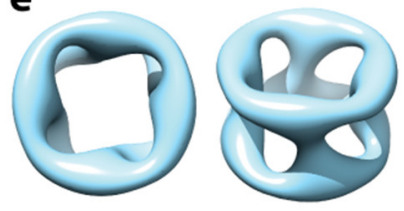

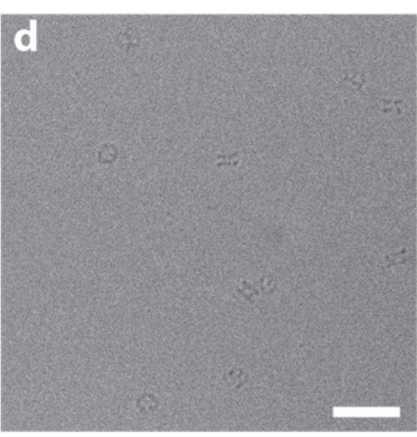

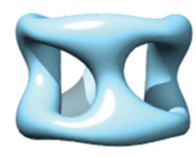

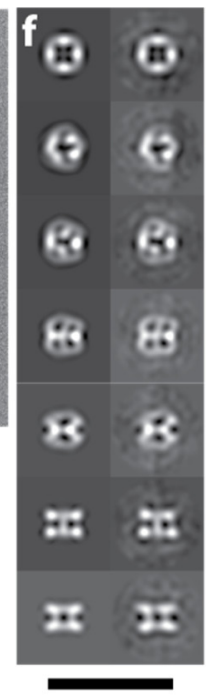

Figure 5 | Tetrameric RNA nanocage. a, A $90^{\circ}$-angle bulge (AACUA) motif ${ }^{55}$ (blue) was added to either beam of a Z-tile to create CZB12b11; this moved both hairpin loops out of the plane of the bulges to create a 3D geometry compatible with the formation of a $13.3 \mathrm{~nm}$ wide tetrameric cage. Numbers indicate the identity of particular bKL interactions that result in $\mathrm{D}_{2}$ symmetry. b, Native PAGE shows the various salt concentrations $\left(\mathrm{Na}^{+}\right.$and/or $\mathrm{Mg}^{2+}$ ) explored to optimize yield of the desired tetramer (target band indicated by a black triangle). c-d, AFM (c) and cryo-EM (d) images of the tetrameric nanocage assembled from CZB12b11. e, Different views of a $13.7 \mathrm{~nm}$ wide structural model generated by cryo-EM single-particle reconstruction. f, Pairwise comparison of representative 2D projections of the 3D reconstruction (left) and corresponding class-averages of individual particles (right). See 


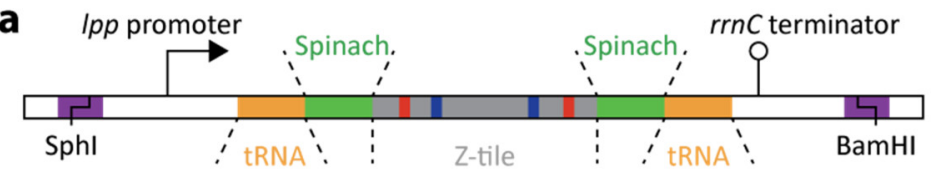

d

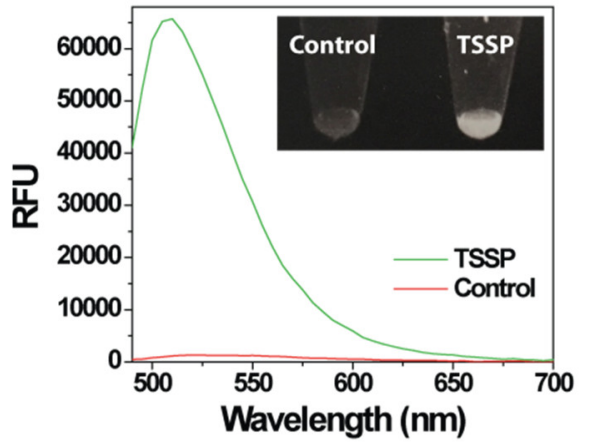

b

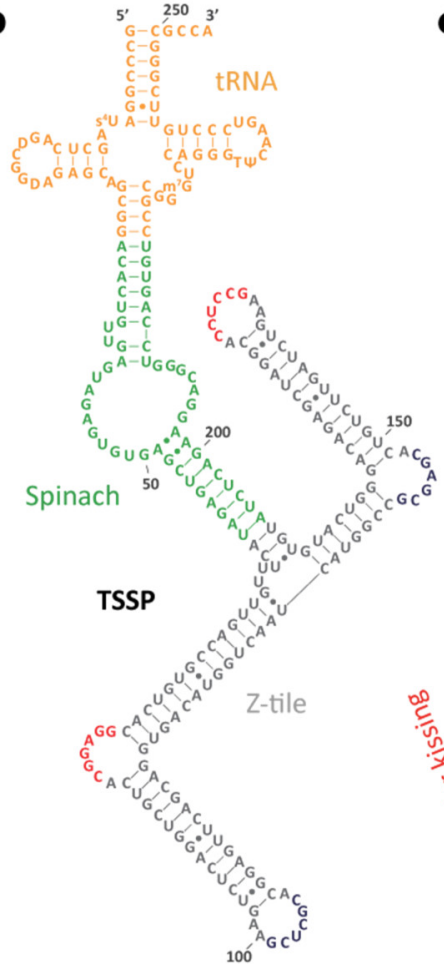

C

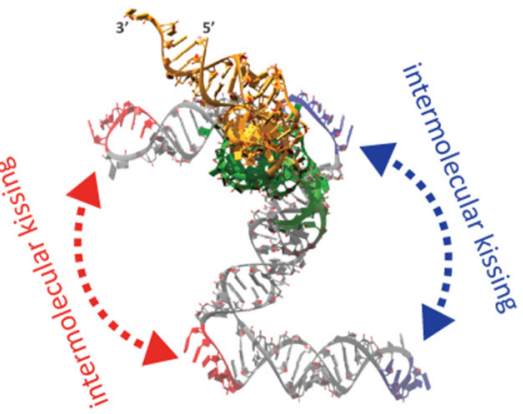

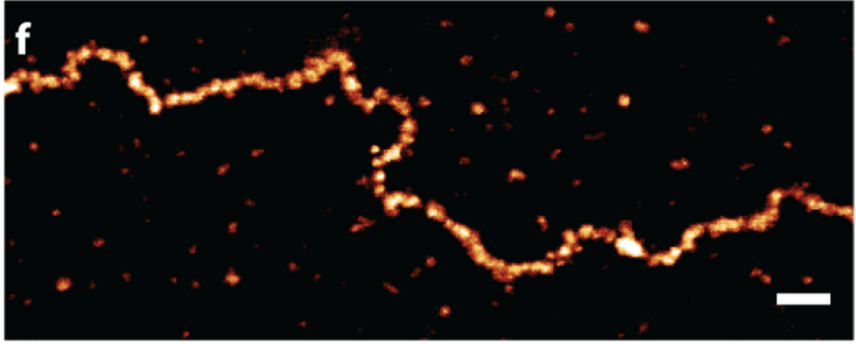

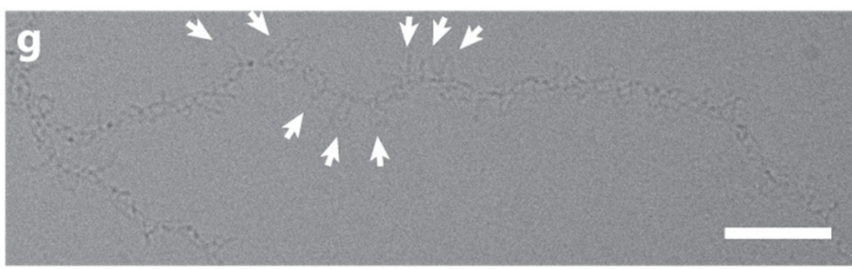

Figure 6 | Cellular production of RNA tiles. a, Design of a gene fragment encoding the RNA construct TSSP, which is a fusion of a tRNA scaffold (orange), a Spinach aptamer (green) and a Z-tile analogous to LZB13 (mostly gray; red and blue segments representing bKL binding regions) except that its bKL sequences were chosen so that every other Z-tile is rotated by $180^{\circ}$ in plane. This gene fragment was inserted between the SphI and BamHI restriction sites of the pUC19 plasmid for the expression in E. coli. b, The predicted secondary structure of TSSP. c, Two views of a 3D model of TSSP built from three PDB structures: a tRNA (ID: 1FIR) ${ }^{65}$, the Spinach aptamer (4KZD) ${ }^{64}$ and the phi29 prohead RNA 3WJ (4KZ2) ${ }^{66}$. d, Emission spectra of bacterial cells with (TSSP) and without (control) plasmid expressing TSSP in the presence of the Spinach fluorophore DFHBI under $469 \mathrm{~nm}$ excitation. Inset: photograph of the same samples with the UV transilluminator and SYBR ${ }^{\circledR}$ Green emission filter in an EC3 bioimaging system (UVP). e, Denaturing PAGE of the total RNA extracted from bacterial cells without (lane 1) and with (lane 2) TSSP-expressing plasmid. A black triangle marks the TSSP band (254 nt). Lane M: DNA size marker. f-g, AFM (f) and cryo-EM (g) images of structures assembled from cell-produced, gel-purified TSSP via in vitro annealing. By cryo-EM, the Spinach/tRNA fusions (indicated by white arrows) could be directly observed, since they caused TSSP ladders to take on a brush-like appearance. Scale bars: $100 \mathrm{~nm}$. 

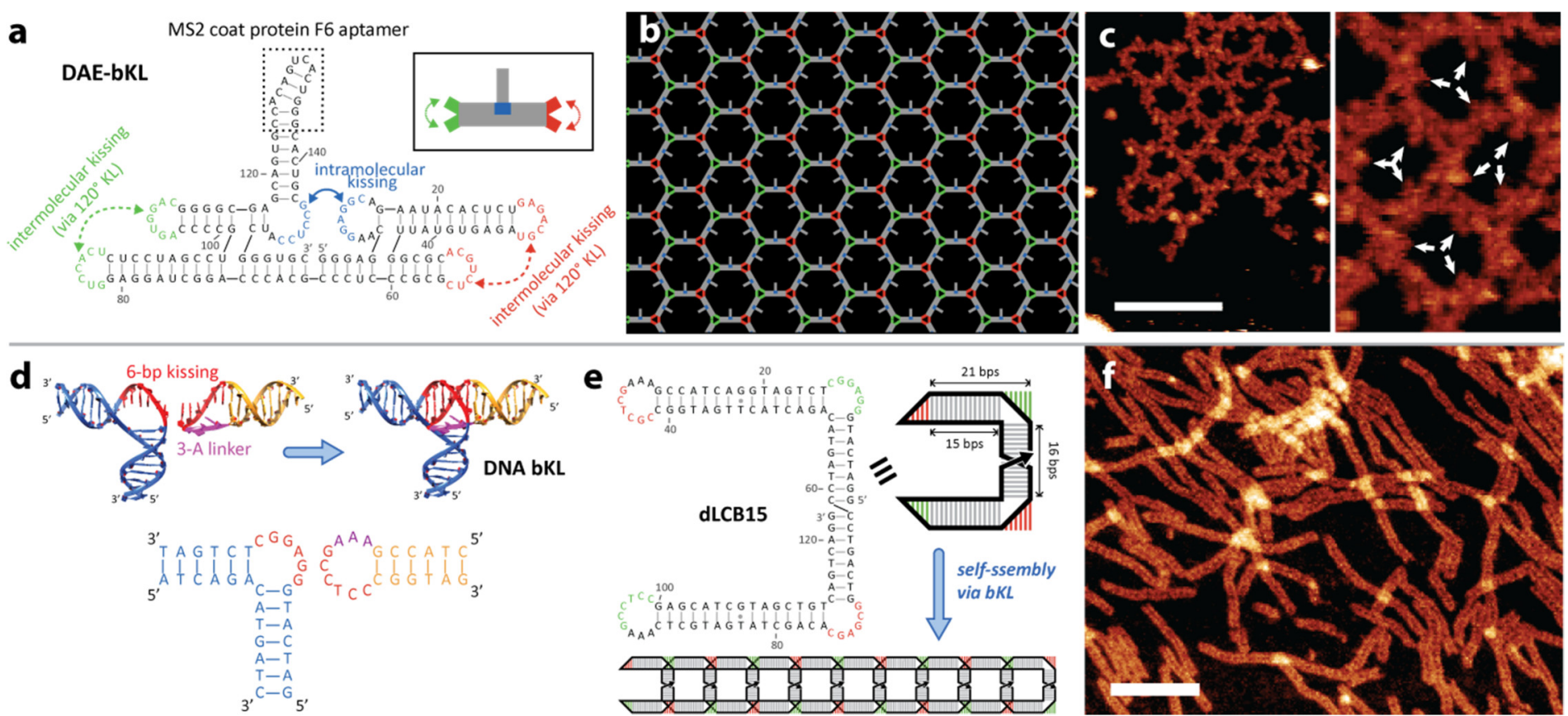

789 Figure 7 | The integration of RNA bKL within RNA origami tile, and its adaption to DNA bKL. a, Insertion of a bKL into a two-helix RNA origami tile (based on a previously reported $2 \mathbf{H}-\mathbf{A E}$ tile $^{22}$, but modified to be one-turn narrower between crossovers)

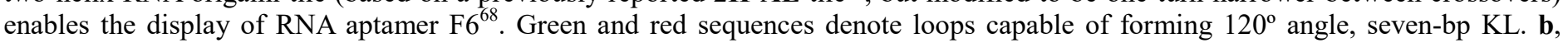
Schematic of the 2D lattice predicted to form from the tile DAE-bKL in (a). c, AFM of lattice cotranscriptionally assembled from DAE-bKL. White arrows show the position of F6 aptamers, pointed into each hexagonal cavity. Scale bar in c: $50 \mathrm{~nm}$. d, Motif fusion is again used to derive the DNA bKL, with the difference that a three dA linker (purple) is used to span the large (23 $\AA$ ) major groove of B-form DNA in the kissing region. e, C-tile designed using the DNA bKL motif, with a symmetry that should minimize curvature. G/T mismatches in this tile derive from an analogous RNA tile sequence. f, AFM of the design from (e) reveals straight ladders. Scale 797 bar in f: $100 \mathrm{~nm}$. 



Supplementary Figure 1| Structural basis for the design of bKL motif. This is a zoom-in view of the stick representations of the $\mathrm{KL}$ and A-form helix models that are shown in Figure 1a. In the KL, the distance between the 3' O of the nucleotide before the two unpaired adenines and the 5' $\mathrm{C}$ of nucleotide after the two unpaired adenines is measured $13.7 \AA$. In the A-form helix, the distance between the 5' $\mathrm{C}$ of the 5'-end nucleotide of one strand and the $3^{\prime} \mathrm{O}$ of the $3^{\prime}$ '-end nucleotide of the other strand is measured $16.7 \AA$. This distance difference of $3 \AA$ can be easily accommodated by the phosphate linkers and also by the structural flexibility of RNA.

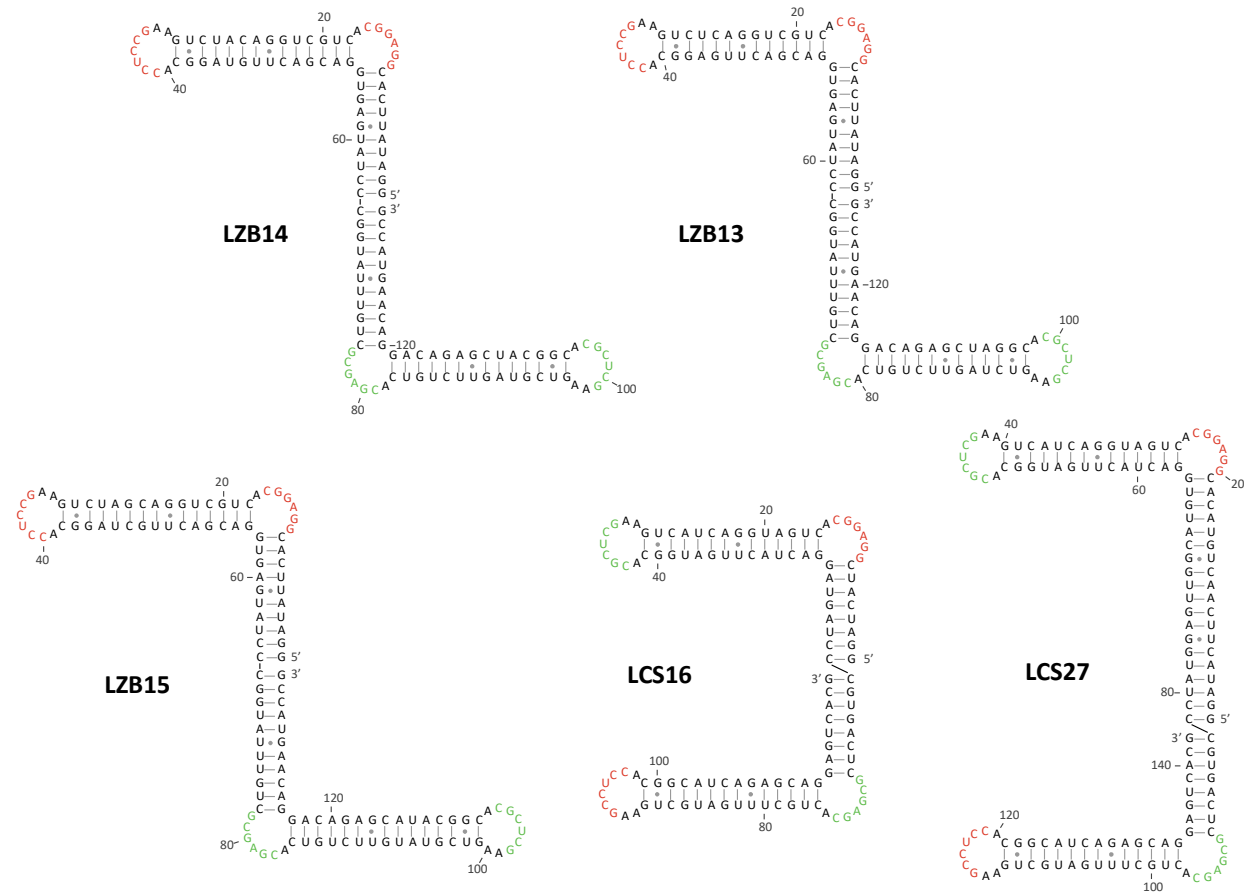

Supplementary Figure 2 | Sequence and predicted secondary structure for the RNA tiles described in Figure 2. Nucleotides participating in kissing interactions are colored as in Figure 2. Tiles are named according to one of two schemes, (Assembled-shape) (Tile-shape) (Beam-description) or (Assembled-shape) (Tile-shape) (Strut-description), where Assembled-shape can be $\mathbf{L}$ (for linear), $\mathbf{R}$ (for ring), or $\mathbf{C}$ (for cage); Tile-shape can be $\mathbf{Z}$ (for Z-shaped tile), or $\mathbf{C}$ (for C-shaped tile); Beam-description can be $\mathbf{B} \boldsymbol{x}$ (for beams having an equal number of base pairs, $x$ ), $\mathbf{B} \boldsymbol{x} / \boldsymbol{y}$ (for beams having two different numbers of base pairs, $x$ and $y$ ), or $\mathbf{B} \boldsymbol{x} \mathbf{b} \boldsymbol{y}$ (for beams having an equal number of base pairs $x+y$, but having a bulge between helix subsegments of length $x$ and $y$ );

Strut-description can be $\mathbf{S} \boldsymbol{x}$ (for a strut containing $x$ base pairs), or $\mathbf{S} \boldsymbol{x} \mathbf{W J}$ (for a strut containing an $x$-way junction). 


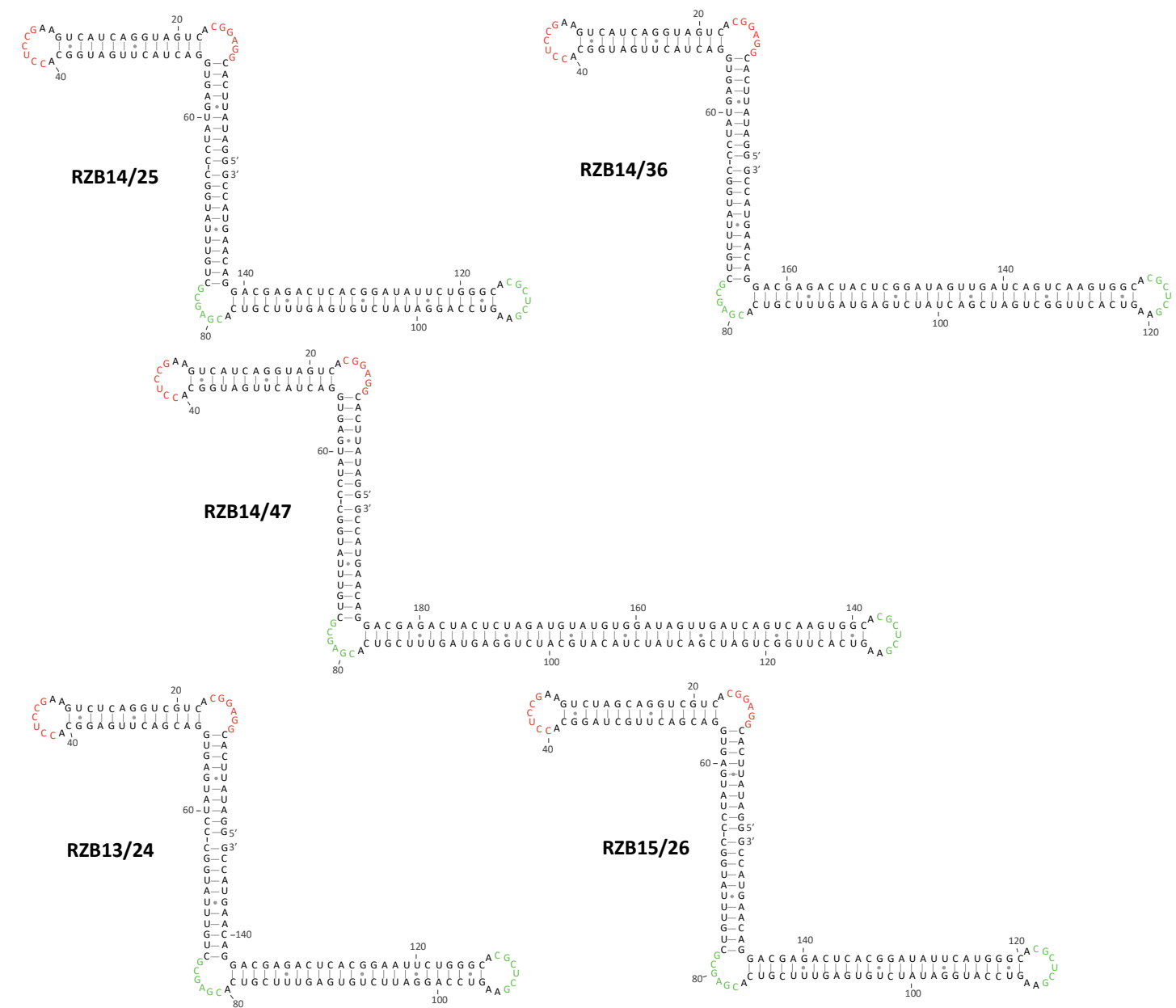

Supplementary Figure 3 | Sequence and predicted secondary structure for the tiles described in Figure 3. 




Supplementary Figure 4| Sequence and predicted secondary structure for the tiles described in Figure 4.

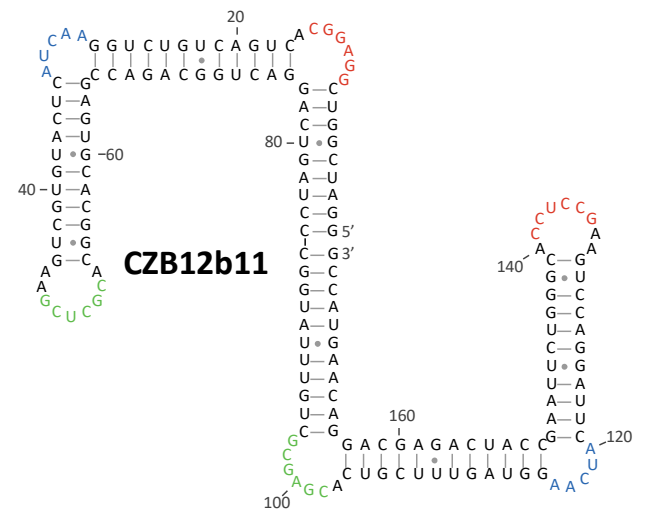

Supplementary Figure 5 | Sequence and predicted secondary structure for the tile CZB12b11, which self-assembles into the tetrameric nanocage described in Figure 5. 


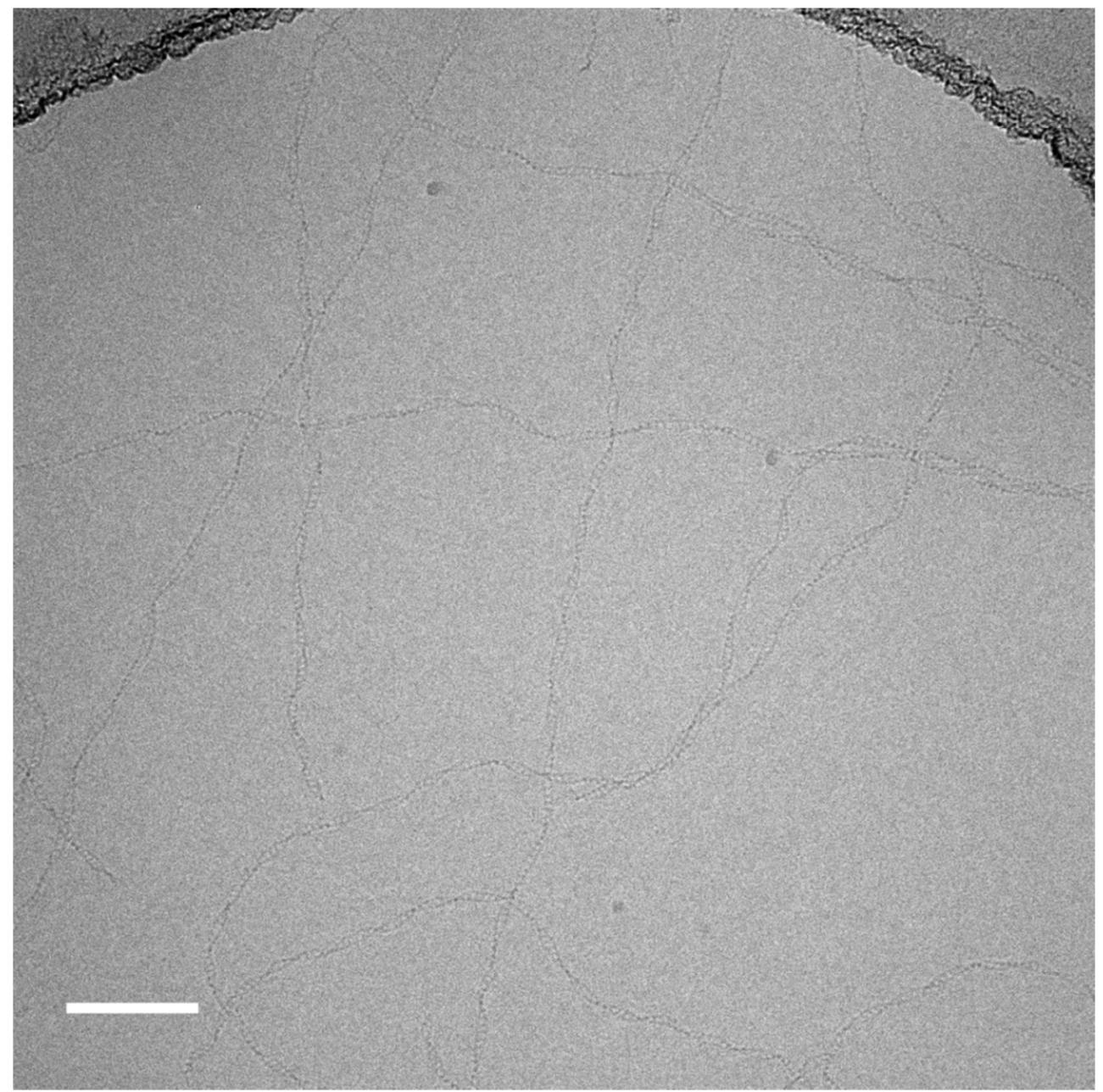

Supplementary Figure 6 | Representative wide-area cryo-EM image showing ladders in vitreous ice suspended across one hole of a holey-carbon grid. The twisted ladders shown were assembled from LZB13. Such cryo-EM images complement results obtained from AFM, which can contain artifacts caused by strong adsorption of structures on the imaging substrate. In contrast, because structures are embedded within a thin layered of vitreous ice, cryo-EM images can better represent the native state of structures and more easily capture features of their 3D geometry, such as twist. Scale bar: $100 \mathrm{~nm}$.
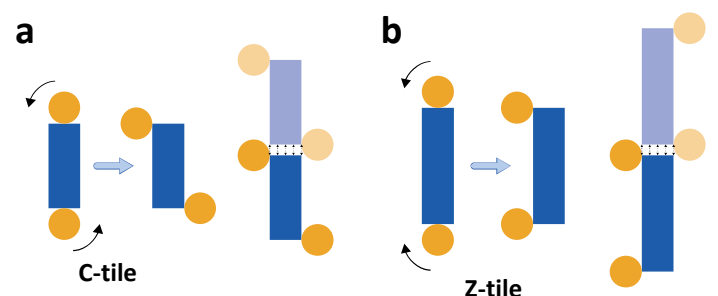

Supplementary Figure 7 | Schematic illustration of possible stacking arrangements for C-tile and Z-tile structures. Here, structures are viewed in cross-section along the axes of the beams; beams are represented by orange circles and struts by blue rectangles. We assume that the flexibility of the bKL may allow the beam to slip sideways, exposing the end of the strut and making it available for stacking. We further assume that dislocation of a beam from a strut has a preferred direction relative to the 3D structure of the bKL, and different tile symmetries exhibit different stacking behaviors. a, For the ladders assembled from the C-tiles beams should slip most easily in opposite directions; thus any two C-tile ladders should be able to stack if they encounter each other without steric hindrance. As noted in the main text, C-tiles assemble with an alternating face-up/face-down symmetry which should minimize curvature and torsion and make the formation of multiple stacking interactions easier, which is consistent with our observation that C-tile ladders stack. b, For the ladders assembled from the Z-tiles, the symmetry of the tiles suggests that to avoid steric hindrance between dislocated rails, stacking would require ladders to be flipped relative to each other to stack. This may decrease the probability of stacking. More important is that Z-tile ladders form rings of variable curvature or twisted ladders, both of which make extended stacking over many struts difficult, which is consistent with our observation that Z-tile structures do not stack. 


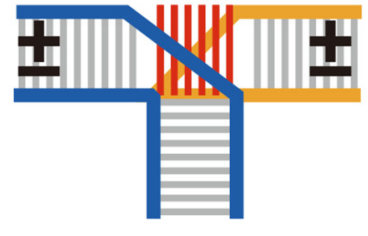

out-of-plane bending

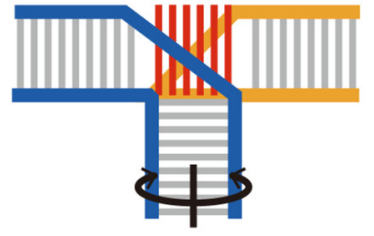

rotation of strut

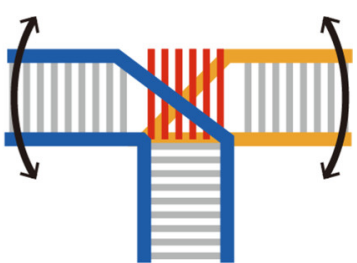

in-plane bending

Supplementary Figure $8 \mid$ Three types of flexibility for the bKL motif. Here we consider motions for which the end of the strut remains centered on the helix segment formed by the kissing interaction, and does not slip. Left, Out-of-plane bending: beams can bend out of the plane defined by the beams and strut ('+' out of the page; '-' into the page). Middle, Rotation of strut: the strut can rotate about its helical axis. In all tiles demonstrated here, we positioned the nick in the phosphate backbone within the strut, which may also contribute to the rotational flexibility of the strut. Right, In-plane bending: the beams can bend in the plane defined by the beams and strut.

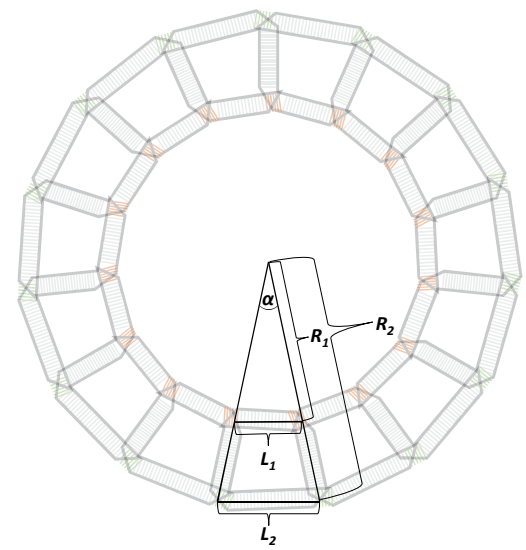

$\frac{L_{1}}{R_{1}}=\frac{L_{2}}{R_{2}}=\frac{L_{2}-L_{1}}{R_{2}-R_{1}}$ $\frac{\alpha}{180^{\circ}} \pi R_{1} \approx L_{1}$

Rotation per tile:

$\alpha \approx \frac{180^{\circ} L_{1}}{\pi R_{1}}=\frac{180^{\circ}}{\pi} \cdot \frac{L_{2}-L_{1}}{R_{2}-R_{1}}$

Number of tiles to complete the ring:

$\frac{360^{\circ}}{\alpha} \approx 2 \pi \frac{R_{2}-R_{1}}{L_{2}-L_{1}}$

Supplementary Figure 9 | Calculating the number of tiles in planar annuli. $L_{2}-L_{l}$ is the length difference between the two beams. $R_{2}-R_{I}$ is the distance between the two points of intersection of the axis of strut and those of the two beams and corresponds to the length of $27.2 \mathrm{bp}$ ( $21 \mathrm{bp}$ for the strut, plus $8.2 \mathrm{bp}$ corresponding to the $2.3 \mathrm{~nm}$ diameter of helix, with a rise of $0.28 \mathrm{~nm} / \mathrm{bp}$ ). Therefore, planar annuli assembled by RZB14/25, RZB14/36, and RZB14/47 (for a hypothetical single-layered annulus) would have 16 or 17

(16.7) tiles, 8 or 9 (8.3) tiles and 5 or 6 (5.6) tiles, respectively. For the double-layered structures assembled from RZB14/47, a total of

32 or 34 tiles (16 or 17 tiles in each of two layers, given that 16.7 was calculated) would be required to create a planar structure.

a

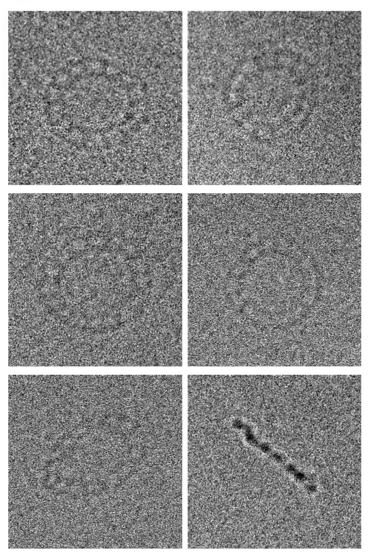

b

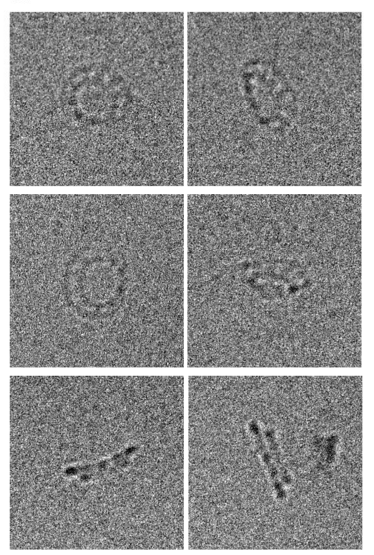

C

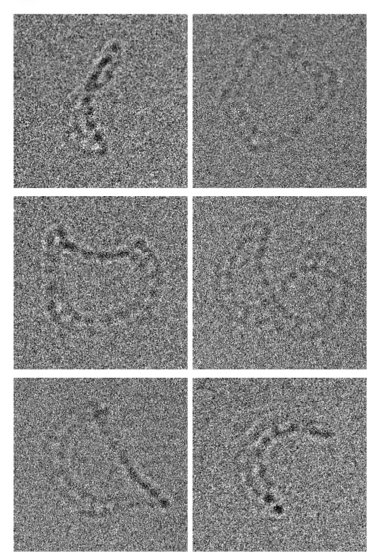

Supplementary Figure 10 | Representative images of annuli assembled from RZB14/25. a, Near-planar annuli comprise 14 to 16 tiles. b, For annuli containing less than 14 tiles, the length difference between beams is too small to accommodate a planar geometry and, consequently, these structures adopt the geometry of a conical frustum. c, For annuli containing more than 16 tiles, the length difference between the beams is too large, so that these structures bend into saddle shapes. All images are $80 \mathrm{~nm} \times 80 \mathrm{~nm}$. 
a

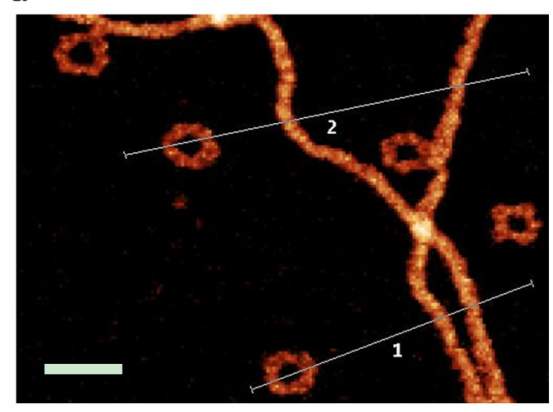

b



C

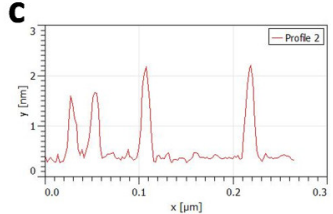

Supplementary Figure 11 | Comparing the heights of the two different kinds of structures self-assembled from RZB14/36 tiles. a, AFM image showing the two different kinds of structures assembled from RZB14/36. Scale bar: $50 \mathrm{~nm}$. b, c, Two extracted height profiles corresponding to the two line segments in a. The single-layered rings (annuli) have a height of 1.2-1.3 nm, which is consistent to the height normally obtained for a single layer of double helix by AFM when scanned in air. In comparison, the long linear structures have a height of 1.6-1.8 nm, consistent with the idea that they are at least partially double layered. Supplementary Fig. 11 has a model of these structures in which one edge of the structure has a single rail, and the other edge has two rails.

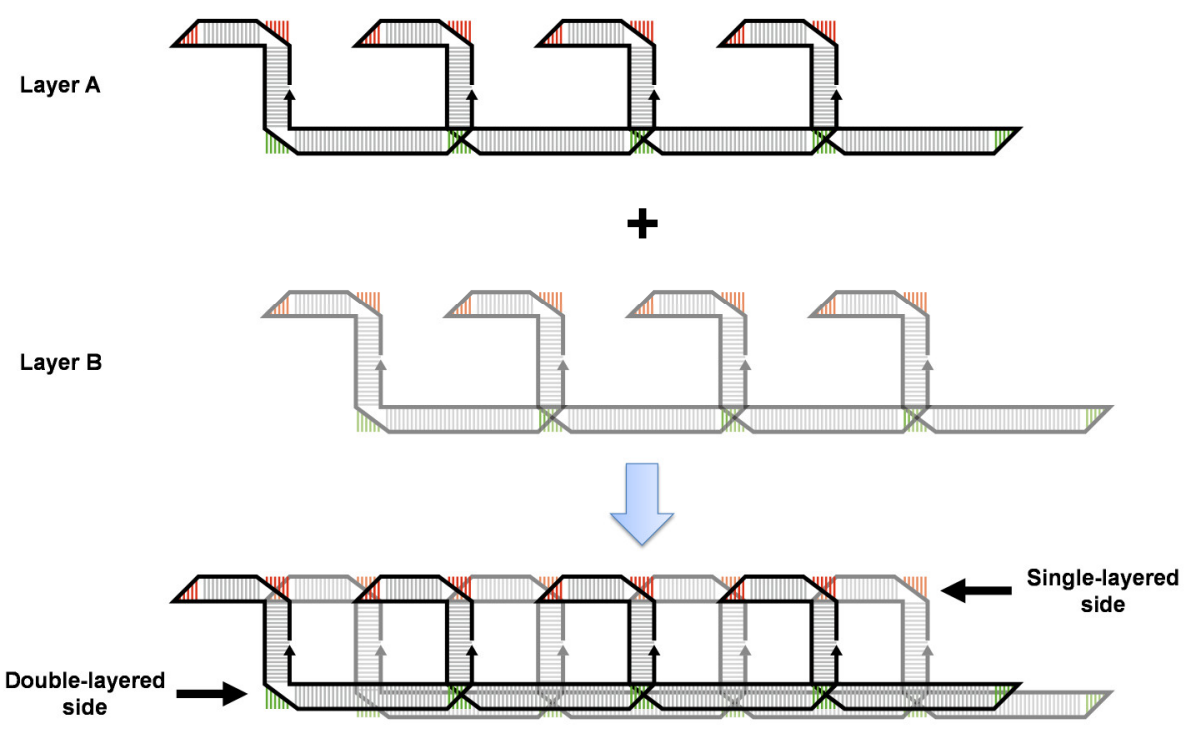

Supplementary Figure 12 | Schematic illustration of the double-layered ladder assembled from RZB14/36. To clarify the structure of the double-layered ladder, we draw two structures (Layer A and Layer B) which can be combined to create the full structure, with the rail formed from the long beams of Layer A on top of the rail formed by the long beams of Layer B. Note that this schematic aims only to clarify the structure - it does not necessarily reflect the assembly pathway, and we think it unlikely that double-layered ladders form from the dimerization of structures such as Layer A and B. Initially, we did not predict these double-layered filaments because our assumptions made them implausible: if the short-beam rail has exactly two turns per repeat unit, as designed, and the tiles are inflexible, then the two long-beam rails would be superimposed on each other, and would be predicted to result in a strong steric clash. In reality, flexibility of the tile may moderate steric hindrance, and bring the energy of the filaments closer to that of the annuli. Further, our analysis of the annuli ignores the persistence length of RNA and the bending energy required to form the inner and outer circles; these factors may increase the energy of the annuli relative to alternative structures like the filaments. 


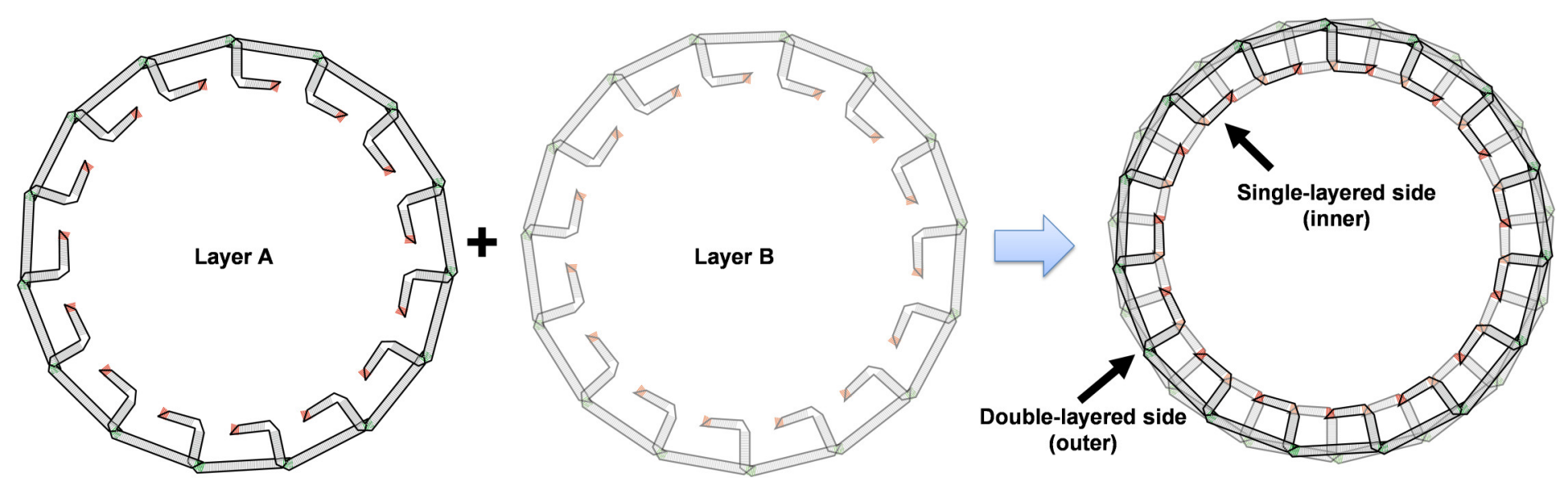

Supplementary Figure 13 | Schematic illustration of the double-layered annulus assembled from RZB14/47 tiles. Similar to the case for double-layered ladders (Supplementary Fig. 11) this schematic is meant only to clarify the structure; it is not meant to suggest that the assembly pathway for the double-layered annulus is via the dimerization of Layer A and Layer B.

\begin{tabular}{|c|c|c|c|c|c|c|c|c|c|c|c|}
\hline$\theta$ & (1) & $\theta$ & (6) & (6) & 6 & (6) & (6) & $\theta$ & 0 & 6 & 6 \\
\hline (6) & (6) & 8 & $\theta$ & $\theta$ & 6 & 6 & (2) & 8 & 9 & 6 & 6 \\
\hline 6 & 8 & $\theta$ & (4) & 89 & 80 & 6 & Q. & 6 & Q & $\theta$ & 6 \\
\hline B & (B) & 8 & (8) & 8 & (8) & 6 & (9) & $\theta$ & (6) & 8 & 13 \\
\hline 83 & 8 & 8 & 8 & 8 & 8. & 9 & 9 & $\theta$ & (3) & 83 & 8 \\
\hline 83 & (6) & 8 & 8 & 8 & (8) & 8 & (8) & 8 & 3 & 8 & 8 \\
\hline 8 & 8 & 8 & 8 & 8 & 8 & 8 & 6 & 8 & 8 & 8 & 3 \\
\hline 7 & 8 & 8 & 8 & 8 & 8 & 8 & 8 & 8 & 8 & 8 & 8 \\
\hline 8 & 8 & 8 & 8 & 8 & 8 & 8 & 8 & 8 & 8 & 8 & 8 \\
\hline 8 & 8 & 8 & 8 & 8 & 8 & 88 & 88 & 8 & 83 & 8 & 88 \\
\hline 8 & 8 & 8 & 8 & 8 & 8 & 8 & 23 & 8 & (35) & 8 & 80 \\
\hline $8 c$ & (8B & 86 & $8 i$ & 86 & 8 & $\approx$ & 86 & 25 & 25 & 25 & 25 \\
\hline 25 & 26 & : & 80 & : & $8 \pi$ & : & 87 & zix & \& & $x$ & 86 \\
\hline z & 25 & 26 & 25 & 25 & 26 & $8 z$ & $8 z$ & ax & dit & ax & is \\
\hline $2 \pi$ & of & z & zi & z & E & 28 & 25 & Is & 26 & : & 8 \\
\hline$\Rightarrow$ & 8 & axi & 28 & $\approx$ & 8 & $\approx$ & 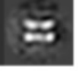 & z & ت3 & $z$ & (75 \\
\hline II & $x$ & x & x & & & & & & & & \\
\hline
\end{tabular}

Supplementary Figure 14 | Complete set of comparisons between 2D projections of a reconstructed 3D model (left) and corresponding class-averages of individual particles (right) for the tetrameric cage made from CZB12b11 tiles. 

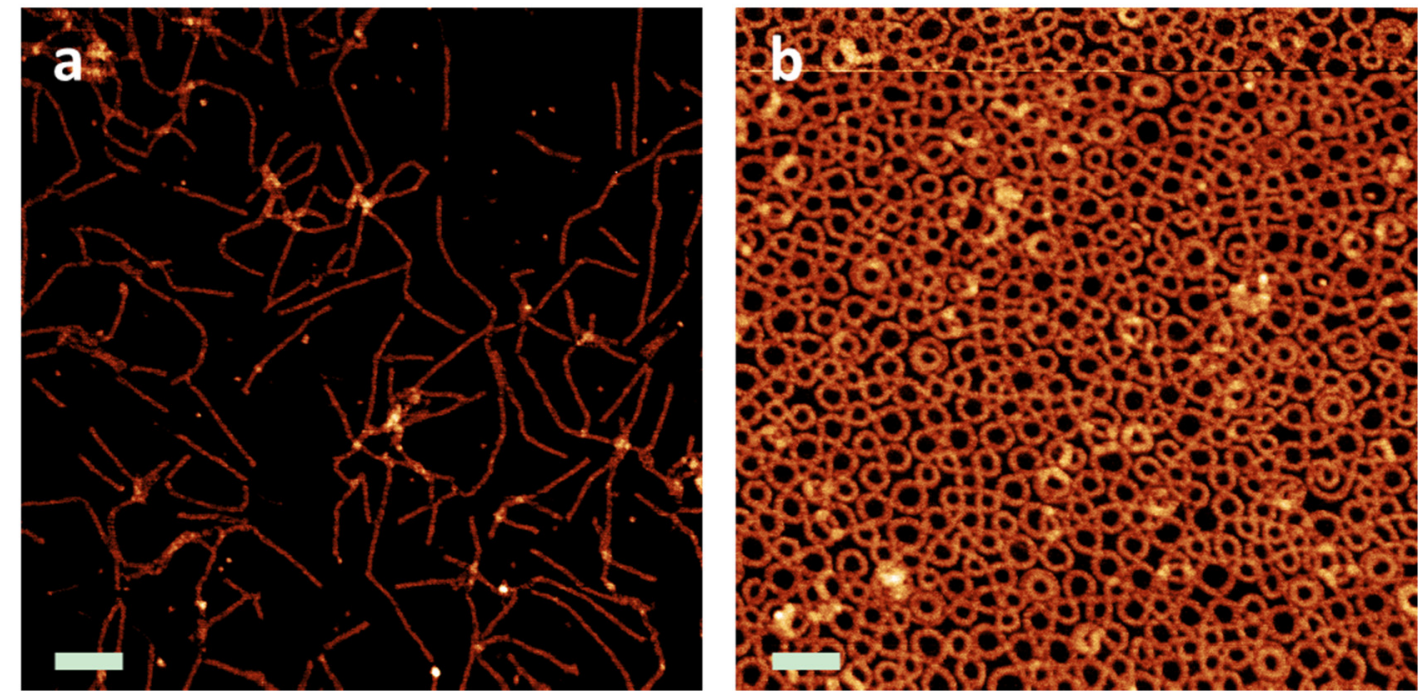

Supplementary Figure 15 | Cotranscriptional assembly of two representative structures. a, AFM image of extended twisted ladders cotranscriptionally assembled from Z-tile LZB13. b, AFM image of the finite-sized annuli cotranscriptionally assembled from Z-tile RZB14/25. Scale bars: $100 \mathrm{~nm}$. 


\begin{tabular}{|c|c|}
\hline & nce \\
\hline \multirow{2}{*}{ LZB13_TMP } & ITCTAATACGACTCACTATAGGATATTCACGGAGGCACTGCTGGACTCTGAAGCCTCCACGGAGTTCAGCAGGTGAGTATCCCGGTATTT \\
\hline & AGCACTGTCTTGATCTGAAGCTCGCACGGATCGAGACAGGACAAGTACCG \\
\hline \multirow{2}{*}{ LZB14_TMP } & TTCTAATACGACTCACTATAGGATATTCACGGAGGCACTGCTGGACATCTGAAGCCTCCAC \\
\hline & 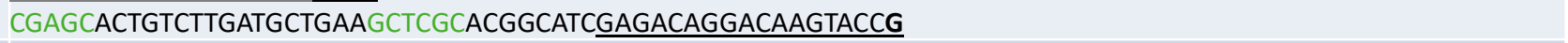 \\
\hline \multirow{2}{*}{ LZB15_TMP } & TTCTAATACGACTCACTATAGGATATTCACGGAGGCACTGCTGGACGATCTGAAGCCTCCACGGATCGTTCAGCAGGTGAGT \\
\hline & CGCGAGCACTGTCTTGTATGCTGAAGCTCGCACGGCATACGAGACAGGACAAGTACCG \\
\hline \multirow[b]{2}{*}{ LCS16_TMP } & ITCTAATACGACTCACTATAGGATCATCGGAGGCACTGATGGACTACTGAAGCTCGCACGGTAGTTCATCAGGATGATCCCGTGACTCGCGAGCAC \\
\hline & $\begin{array}{l}\text { TGCTTTGATGCTGAAGCCTCCACGGCATCAGAGCAGGAGTCACG GGCCGGCATGGTCCCAGCCTCCTCGCTGGCGCCGGCTGGGCAACATGCTT } \\
\text { CGGCATGGCGAATGGGAC }\end{array}$ \\
\hline \multirow[b]{2}{*}{ LCS27_TMP } & ITCTAATACGACTCACTATAGGATACTTCAACTGTACACGGAGGCACTGATGGACTACTGAAGCTCGCACGGTAGTTCATCAGGTGTACGGTTGAG \\
\hline & $\begin{array}{l}\text { GTATCCCGTGACTCGCGAGCACTGCTTTGATGCTGAAGCCTCCACGGCATCAGAGCAGGAGTCACG GGCCGGCATGGTCCCAGCCTCCTCGCTG } \\
\text { GCGCCGGCTGGGCAACATGCTTCGGCATGGCGAATGGGAC }\end{array}$ \\
\hline \multirow{2}{*}{ RZB14/25_TMP } & TTCTAATACGACTCACTATAGGATATTCACGGAGGCACTGATGGACTACTGAAGCCTCCACGGTAGTTCATC \\
\hline & GAGCACTGCTTTGAGTGTCTATAGGACCTGAAGCTCGCACGGGTCTTATAGGCACTCAGA \\
\hline \multirow{3}{*}{ RZB14/36_TMP } & AGGATATTCA \\
\hline & GAGCACTGCTTTGATGAGTCTATCAGCTAGTCGGTTCACTGAAGCTCGCACGGTGAACTGACTAGTTGATAGGCTCATCAGAGCAGGACAAGTAC \\
\hline & CCCAGCCTCCTCGCTGGCGCCGGCTGGGCAACATGCTTCGGCATC \\
\hline \multirow{3}{*}{ RZB14/47_TMP } & TTCTAATACGACTCACTATAGGATATTCACGGAGGCACTGATGGACTAC \\
\hline & GAGCACTGCTTTGATGAGGTCTACGTACATCTATCAGCTAGTCGGTTCACTGAAGCTCGCACGGTGAACTGACTAG \\
\hline & TACCGGGCCGGCATGGTCCCAGCCTC \\
\hline \multirow{2}{*}{ RZB13/24_TMP } & ITCTAATACGACTCACTATAGGATATTCACGGAGGCACTGCTGGACTCTGAAGCCTCCACGGAGTTCAGCAGGTGAGTATCCCGGTATTTGTCGCG \\
\hline & AGCACTGCTTTGAGTGTCTTAGGACCTGAAGCTCGCACGGGTCTTAAGGCACTCAGAGCAGGACAAGTACCG \\
\hline \multirow{2}{*}{ RZB15/26_TMP } & ITCTAATACGACTCACTATAGGATATTCACGGAGGCACTGCTGGACGATCTGAAGC \\
\hline & CGCGAGCACTGCTTTGAGTGTCTATAGGTACCTGAAGCTCGCACGGGTACTTATAG \\
\hline \multirow{3}{*}{ CZB12b11_TMP } & ITCTAATACGACTCACTATAGGATCGGTCGGAGGCACTGACTGTCTGGAACTACT \\
\hline & GGACTGATCCCGGTATTTGTCGCGAGCACTGCTTTGATGGAACTACTTAGGACCT \\
\hline & $\underline{\text { ACCG }}$ \\
\hline \multirow{3}{*}{ LCS3WJ_TMP } &  \\
\hline & ATCGCATCCGACGACACGCGAGCACTTGTATGTAGATGAAGCTCGCACGTCTACGTACAAGGTGTCGTTGGATGCGGATCCCGTGACTCGGAGGC \\
\hline & $\begin{array}{l}\text { ACTGCTTTGATGCTGAAGCCTCCACGGCATCAGAGCAGGAGTCACGGGCCGGCATGGTCCCAGCCTCCTCGCTGGCGCCGGCTGGGCAACATG } \\
\text { CTTCGGCATGGCGAATGGGAC }\end{array}$ \\
\hline \multirow{4}{*}{ LCS4WJ_TMP } & ITCTAATACGACTCACTATAGGATACCTGTCAATCGCTGCGTCCACACTCACGTGTTGCTGAAGTGGACACGGCAACGCGTGAGGCAGCGGTTGA \\
\hline & CACCATACCGACGACACGCGAG CACTTGTATGTAGATGAAGCTCGCACGTCTACGTACAAGGTGTCGTTGGTATGGACTGAATGTGGCC GTACGC \\
\hline & AATCATGTTAGATTGAA GCGTACACGATCTAGCATGATGGCCACGTTCAGTGGTATCCCGTGACTCGGAGGCACTGCTTTGATGCTGAAGCCTCCA \\
\hline & CGGCATCAGAGCAGGAGTCACG GGCCGGCATGGTCCCAGCCTCCTCGCTGGCGCCGGCTGGGCAACATGCTTCGGCATGGCGAATGGGAC \\
\hline \multirow{3}{*}{ LZB19/47_TMP } & ITCTAATACGACTCACTATAGGATATTCACGGAGGCACTGATGGTCTAGCGTACTGAAGCCTCCACGGTACGTTAGACTATCAGGTGAGTATCCCG \\
\hline & GTATTTGTCGCGAGCACTGCTTTGATGAGGTCTACGTACATCTATCAGCTAGTCGGTTCACTGAAGCTCGCACGGTGAACTGACTAGTTGATAGGT \\
\hline & $\begin{array}{l}\text { GTATGTAGATCTCATCAGAGCAGGACAAGTACCG GGCCGGCATGGTCCCAGCCTCCTCGCTGGCGCCGGCTGGGCAACATGCTTCGGCATGGCG } \\
\text { AATGGGAC }\end{array}$ \\
\hline \multirow{3}{*}{ LZB10/47_TMP } & ITCTAATACGACTCACTATAGGATATTCACGGAGGCACTGACATCTGAAGCCTCCACGGATGTCAGGTGAGTATCCCGGTATTTGTCGCGAGCACT \\
\hline & GCTTTGATGAGGTCTACGTACATCTATCAGCTAGTCGGTTCACTGAAGCTCGCACGGTGAACTGACTAGTTGATAGGTGTATGTAGATCTCATCAG \\
\hline & AGCAGGACAAGTACCGGGCCGGCATGGTCCCAGCCTCCTCGCTGGCGCCGGCTGGGCAACATGCTTCGGCATGGCGAATGGGAC \\
\hline
\end{tabular}

Supplementary Table 1 | Sequences of DNA templates for the run-off transcription of RNA strands for the Z- or C-tiles. All sequences are written from 5' to 3'. Sequences have been colored to match schematic illustrations elsewhere in this paper. In each sequence, a pair of bold Gs mark the start and end of transcription. Thus the sequence of each transcribed RNA is simply the sequence which runs from the first bold $\mathrm{G}$ to the second bold G, with all T's replaced by U's. Primer-binding regions for PCR amplification are underlined. The forward primer is the 5' underlined sequence; reverse primer is the reverse complement of the 3' underlined sequence. To reduce transcriptional heterogeneity at the 3 ' end, the first two nucleotides of all reverse primers were modified with a 2'-OMe group. In the process of ordering template sequences as gBlocks gene fragments from IDT, we found that adding extra, unrelated sequence to the 3' end of templates increased the probability of successful synthesis. Thus, some of the DNA sequences include a sequence encoding the HDV ribozyme (colored in grey) at their 3' end; however, this appended sequence was not amplified in subsequent PCR and was therefore not present in the resulting RNA transcripts. 


$\begin{array}{lll}\text { Strand Name } & \text { Sequence } \\ \text { DAE-bKL_tMP } & \text { TTCTAATACGACTCACTATAGGGAGCAAGGAGGCAGAATACACTCTGAGACGTAGAGTGTATTGGCGCACGTCTCGCGCCCTCCCGCACCCAGGC } \\ \text { DAE-bKL_FWD } & \text { TTCTAATACGACTCACTATAGGG } \\ \text { DAE-bKL_REV } & \text { GCACCCGATGGAGGCGCAGTGCCCAGTGACTGTGGCAC }\end{array}$

Supplementary Table 2 Sequences of strands used to synthesize the single-stranded RNA origami tile DAE-bKL. DAE-bKL_TMP is the PCR template; DAE-bKL_FWD and DAE-bKL_REV are the two PCR primers. Note that DAE-bKL_TMP does not include all of the template sequence required for the transcription of the RNA tile: the last 20 nt of the RNA sequence is provided by the reverse primer (DAE-bKL_REV).

\begin{tabular}{|c|c|}
\hline \multirow{2}{*}{ Strand Name } & Sequence \\
\hline & GTCAACATGCATGCTCGAGGTCGCCCCATCAAAAAAATATTCTCAACATAAAAAACTTTGTGTAATACTTGTAACGCTGAATTCGCCCG GATAGCTC \\
\hline \multirow{4}{*}{ TSSP_TMP } & AGTCGGTAGAGCAGCGGACACTGTTGAGTAGAGTGTGAGCTGAGATACTTTGTTGACCGTGTCACGGAGGCACTGCTGGACTCTGAAGCTCGC \\
\hline & ACGGAGTTCAGCAGGTGACATGGTCAATCATGGCCGCGAGCACTGTCTTGATCTGAAGCCTCCACGGATCGAGACAGGGTCATGTGTATCTCAG \\
\hline & AAGGACGGGTCCAGTGTCCGCG GGTCCAG G GTTCAAGTCCCTGTTCG GGCGCCACTGCAGATCCTTAGCGAAAGCTAAGGATTTTTTTTAAGCT \\
\hline & TGATGGGGGCTTAGGATCCGGAGCTCCA \\
\hline TSSP_FWD & GTCAACATGCATGCTCGAGGT \\
\hline TSSP REV & TGGAGCTCCGGATCCTAAGC \\
\hline
\end{tabular}

Supplementary Table 3 | Sequences of strands used to construct the gene for TSSP. The DNA template (TSSP_TMP) was PCR-amplified with the two primers (TSSP_FWD and TSSP_REV). Then the PCR product was digested with BamH I and Sph I and cloned into the BamH I + Sph I sites of vector pUC19. In the DNA template, the primer-binding regions are underlined and the lpp promoter and $r r n C$ terminator regions are italicized; the first nucleotide to be transcribed (a G) appears in bold.

\begin{tabular}{ll} 
Strand Name & Sequence \\
\hline dLCB15_A & GGATCATGGGAGGCTCTGATGGACTACCGAAAGCTCGCCGGTAGTTCATCAGACA \\
dLCB15_B & /Phos/TGATCCCCTGACTGGCGAGCACAGCTATGATGCTCAAAGCCTCCGAGCATCGTAGCTGTCAGTCAGG \\
dLCB15_Splint & GCTCGCCAGTCAGGGGATCATGTCTGATGAACTACCGGCGAGC
\end{tabular}

Supplementary Table 4 | Sequences of strands used to synthesize the DNA bKL-based tile dLCB15 via ligation. Tile precursor strands dLCB15_A and dLCB15_B were hybridized with the splint strand dLCB15_Splint and ligated to form the complete dLCB15 tile. The strand dLCB15_B is phosphorylated on its 5' end and thus is prefixed by /Phos/. Complementary regions between the precursor strands and the splint strand are underlined with wavy and straight lines. 
This document contains the wide-area AFM and cryo-EM images for the structures constructed in this work.

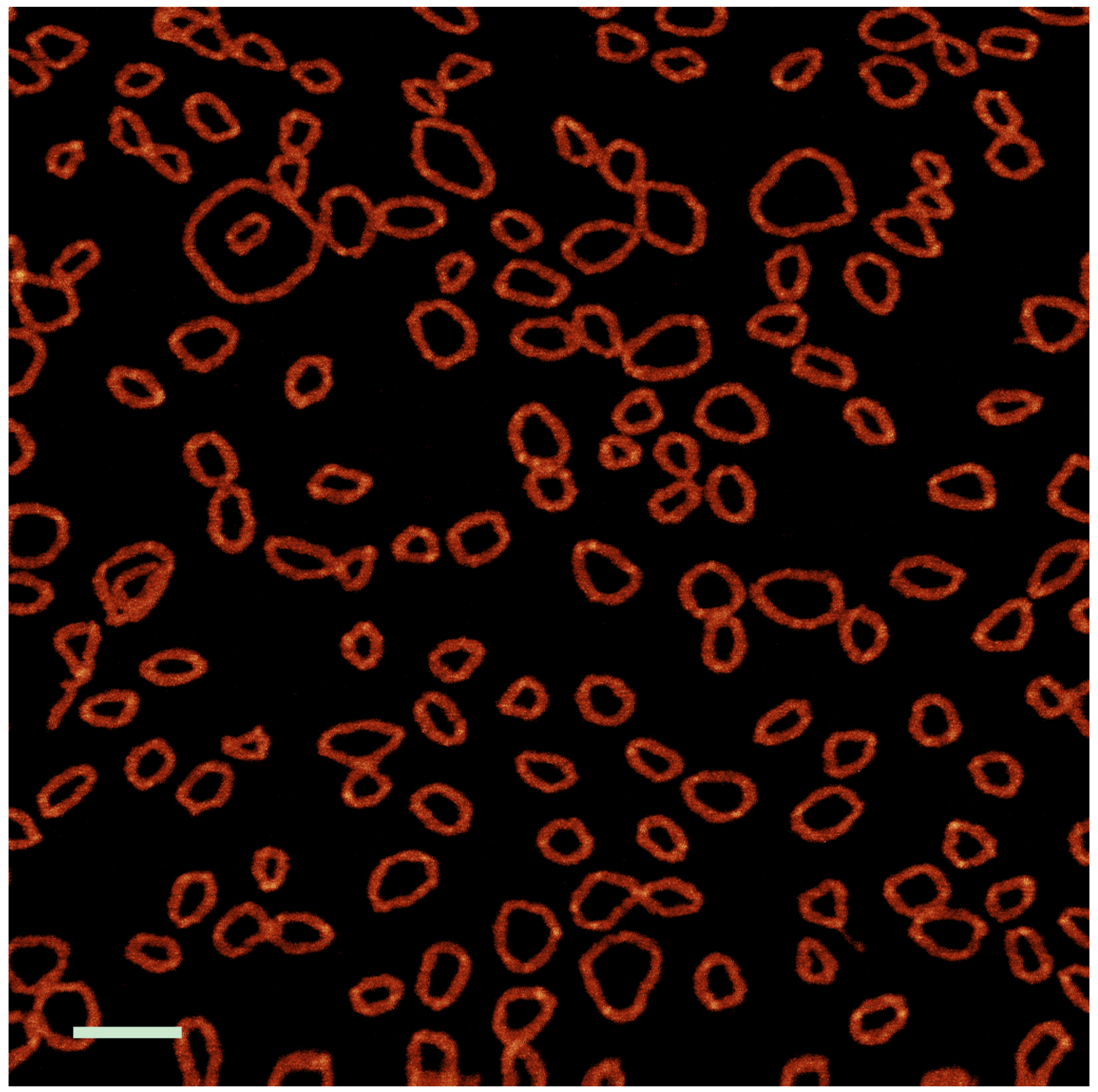

Supplementary Figure 16 | Wide-area AFM image of the structure assembled from Z-tile LZB14. The corresponding small-area image is Figure 2b. Scale bar is $100 \mathrm{~nm}$. 




Supplementary Figure 17 | Wide-area cryo-EM image of the structure assembled from Z-tile LZB14. The corresponding small-area image is Figure 2c. Scale bar is $100 \mathrm{~nm}$. 


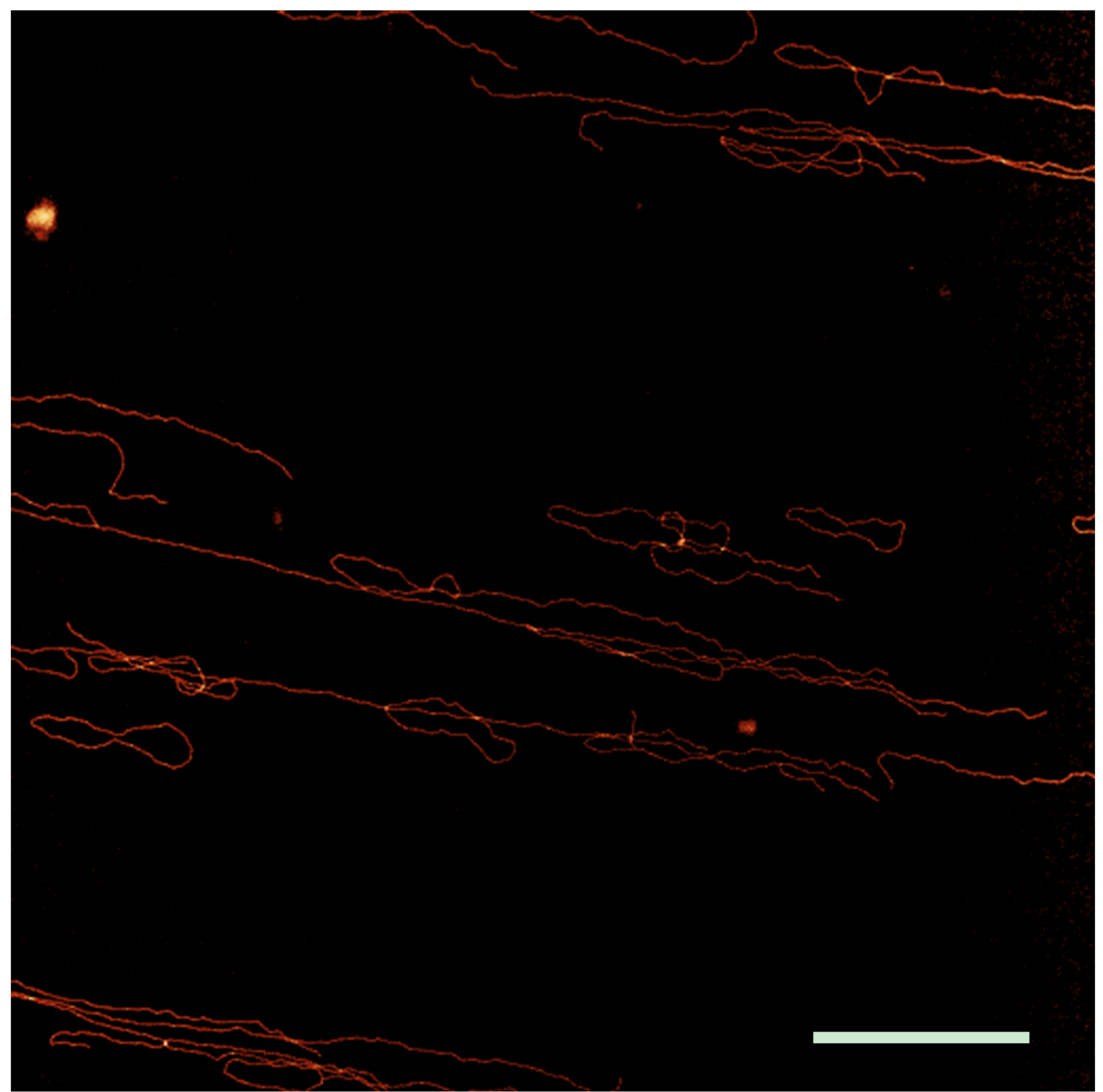

Supplementary Figure 18 | Wide-area AFM image of the structure assembled from Z-tile LZB13. The corresponding small-area image is Figure 2f. Scale bar is $1 \mu \mathrm{m}$. The wide-area cryo-EM image is shown in Supplementary Figure 6. 


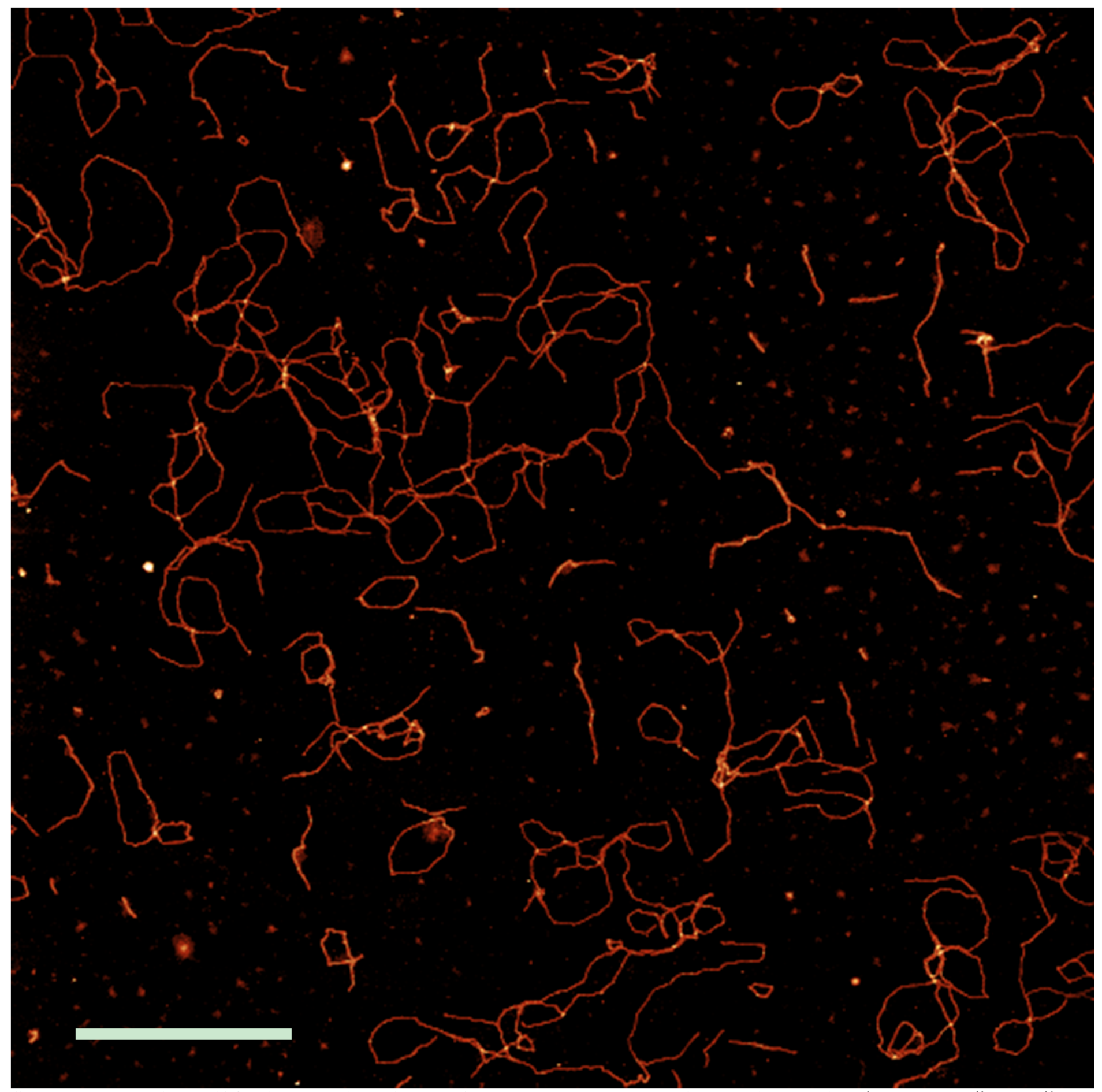

Supplementary Figure 19 | Wide-area AFM image of the structure assembled from Z-tile LZB15. The corresponding small-area image is Figure 2h. Scale bar is $1 \mu \mathrm{m}$. 


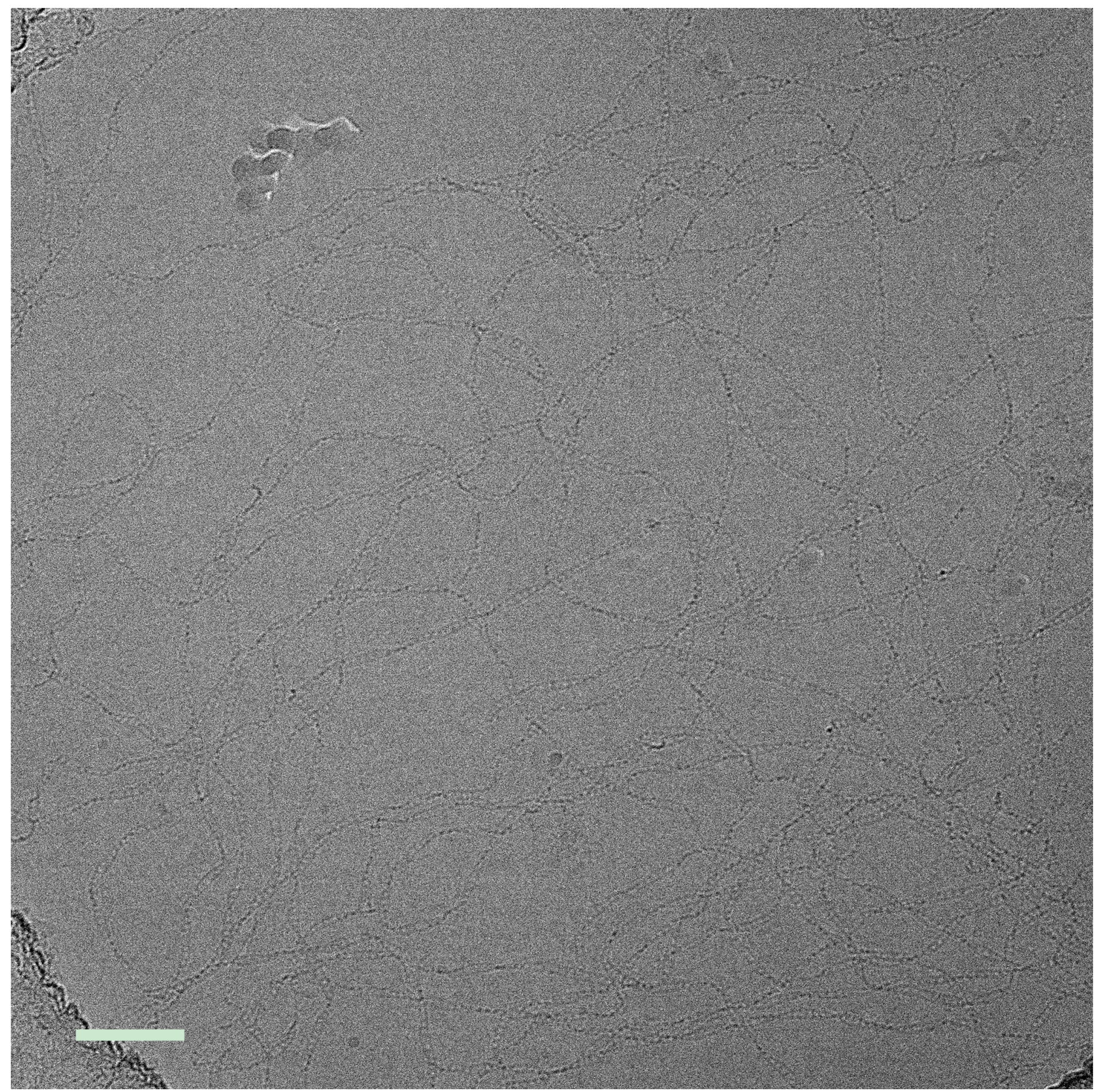

Supplementary Figure 20 | Wide-area cryo-EM image of the structure assembled from Z-tile LZB15. The corresponding small-area image is Figure 2i. Scale bar is $100 \mathrm{~nm}$. 


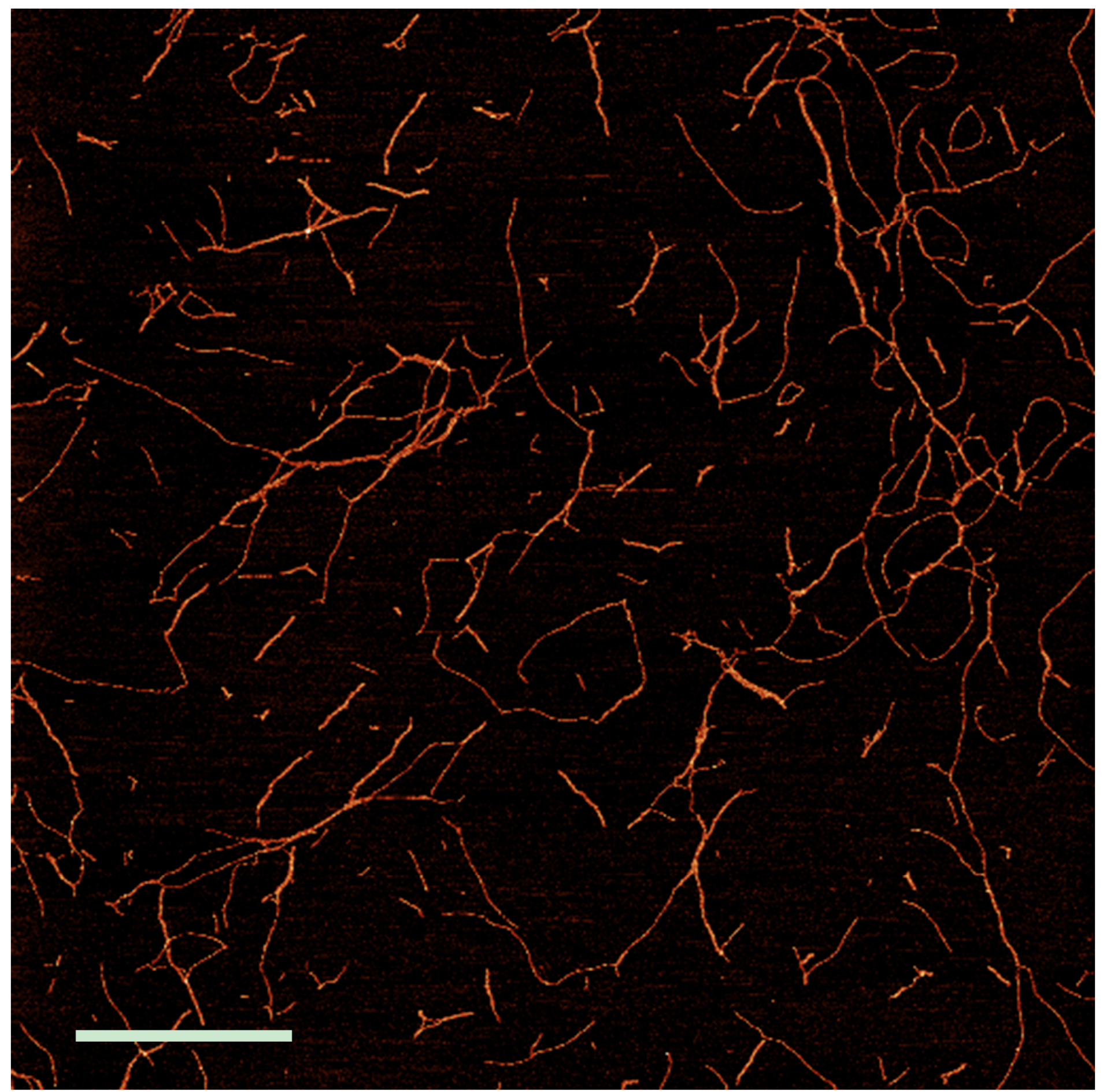

Supplementary Figure 21 | Wide-area AFM image of the structure assembled from C-tile LCS16. The corresponding small-area image is Figure $2 \mathrm{k}$. Scale bar is $1 \mu \mathrm{m}$. 




Supplementary Figure 22 | Wide-area cryo-EM image of the structure assembled from C-tile LCS16. The corresponding small-area image is Figure 21. Scale bar is $100 \mathrm{~nm}$. 


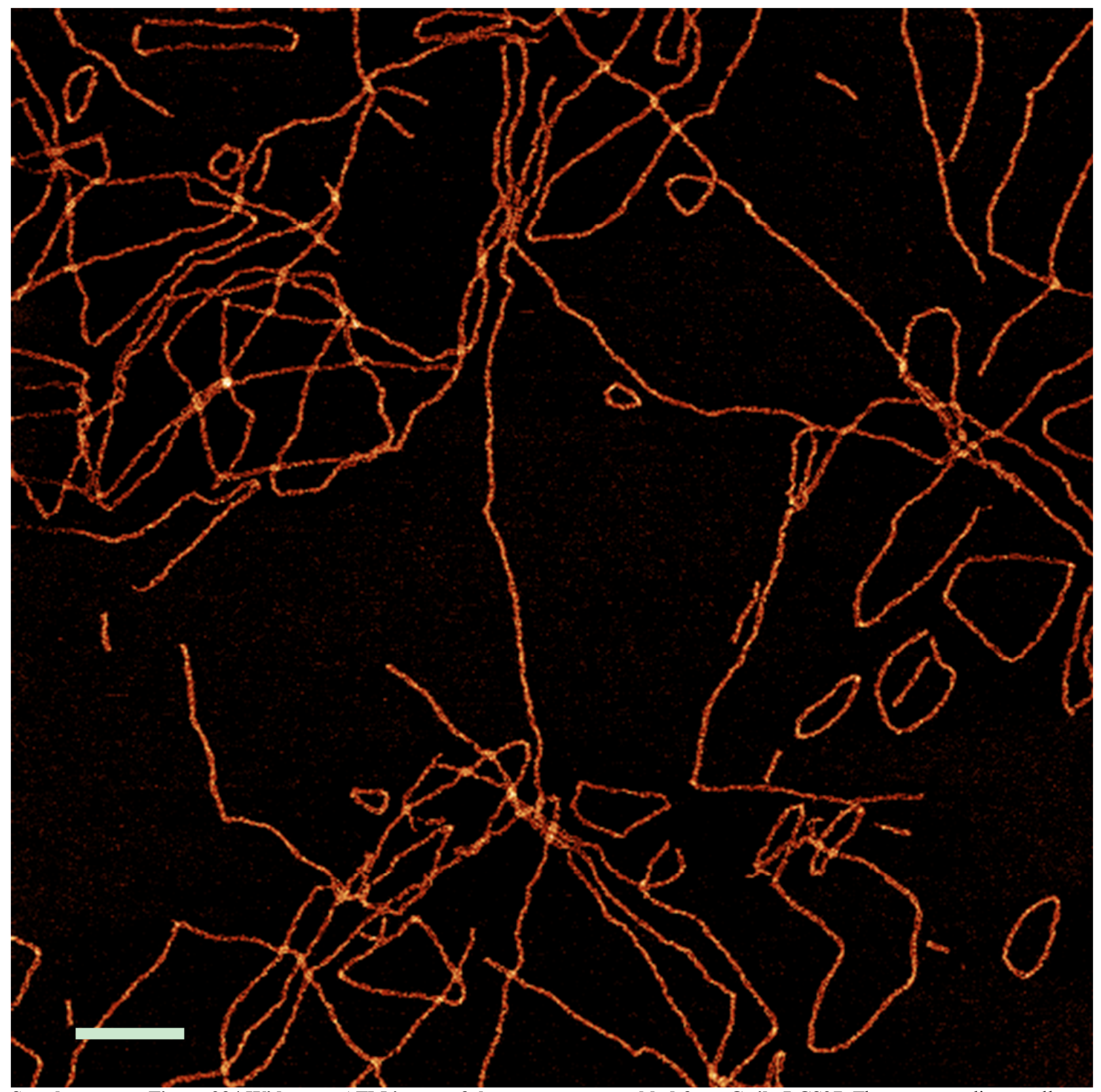

Supplementary Figure 23 | Wide-area AFM image of the structure assembled from C-tile LCS27. The corresponding small-area image is Figure $2 \mathrm{~m}$. Scale bar is $200 \mathrm{~nm}$. 


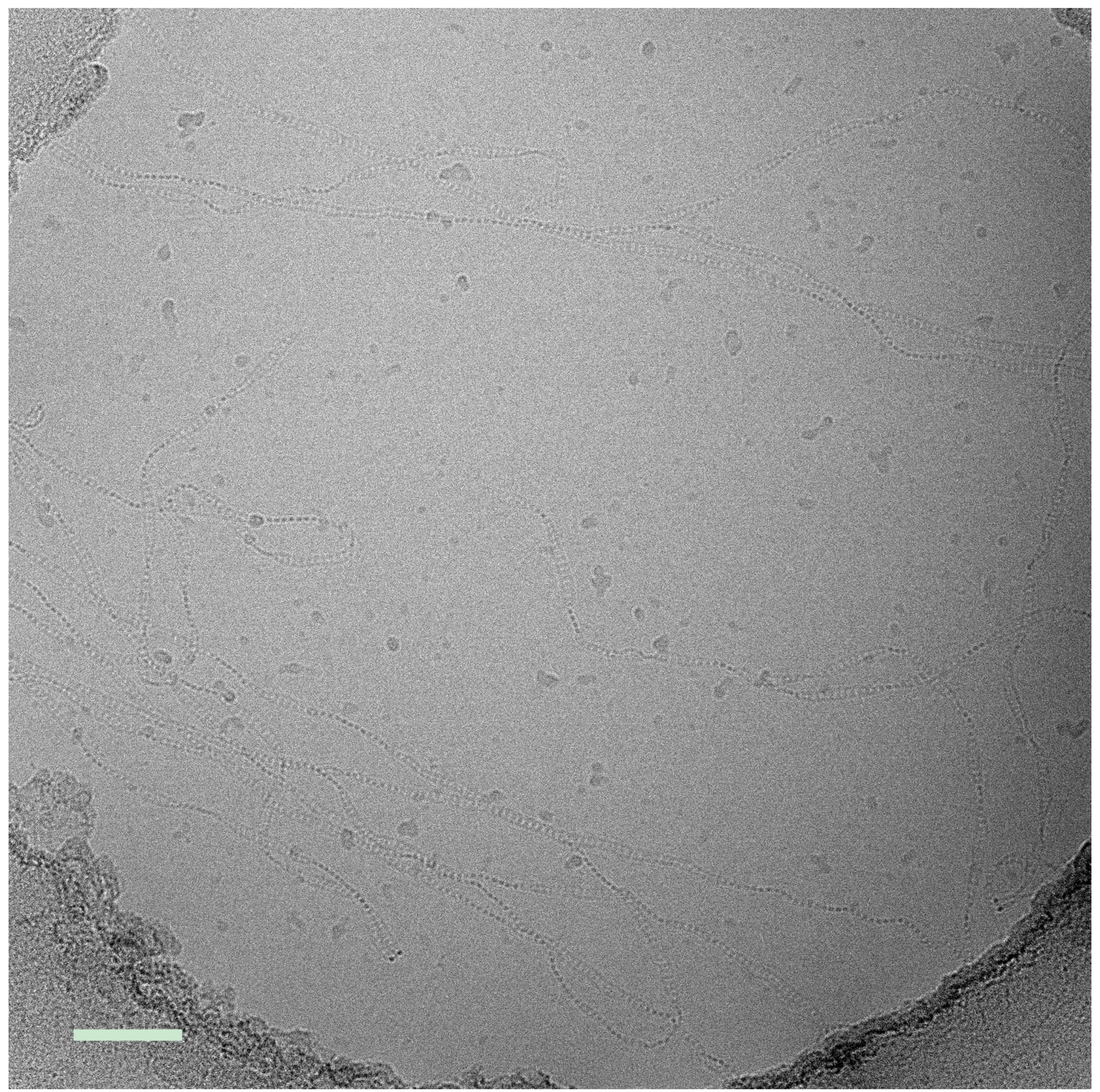

Supplementary Figure 24 | Wide-area cryo-EM image of the structure assembled from C-tile LCS27. The corresponding small-area image is Figure 2n. Scale bar is $100 \mathrm{~nm}$. 


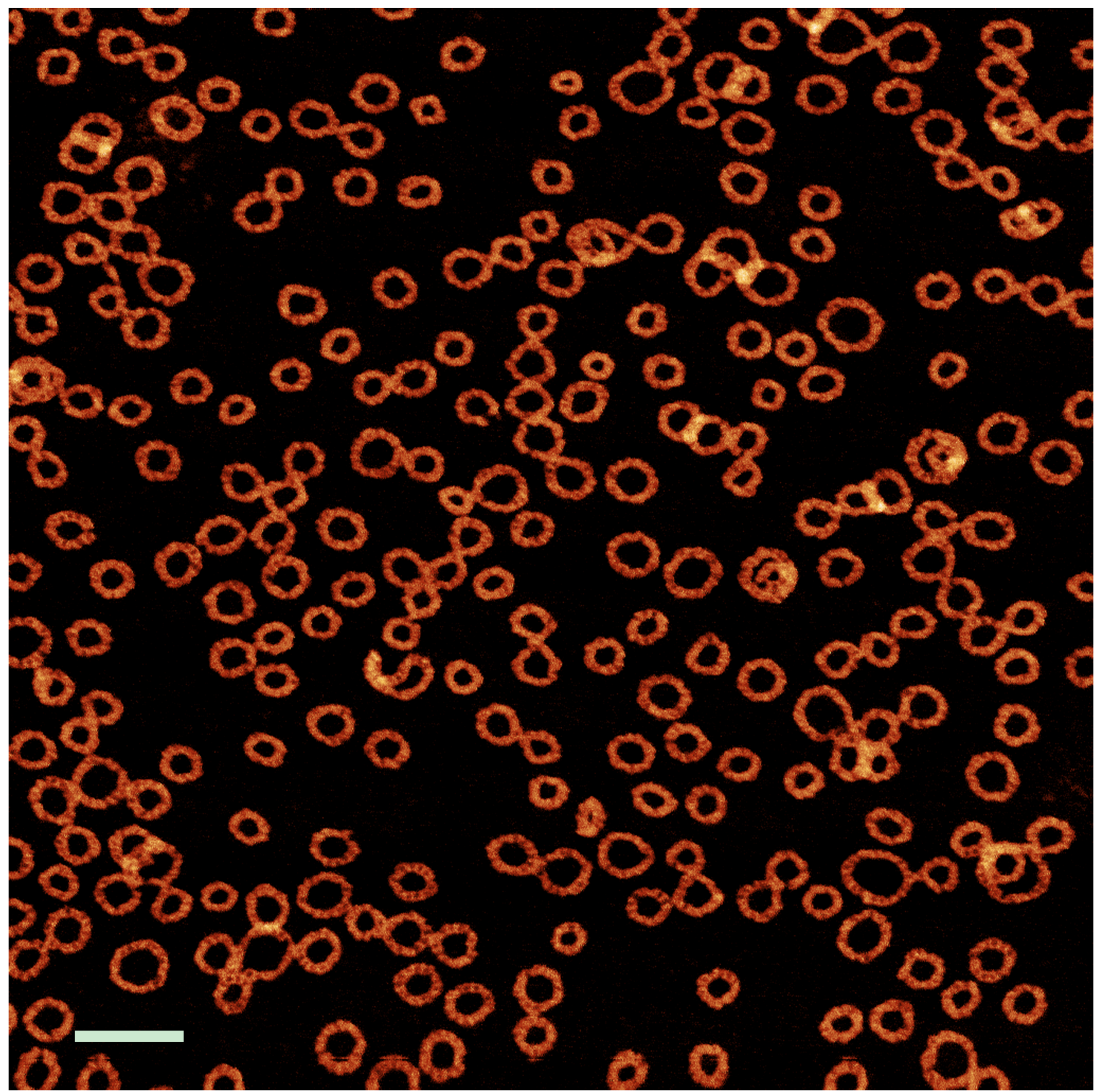

Supplementary Figure 25 | Wide-area AFM image of the structure assembled from Z-tile RZB14/25. The corresponding small-area image is Figure 3b. Scale bar is $100 \mathrm{~nm}$. 


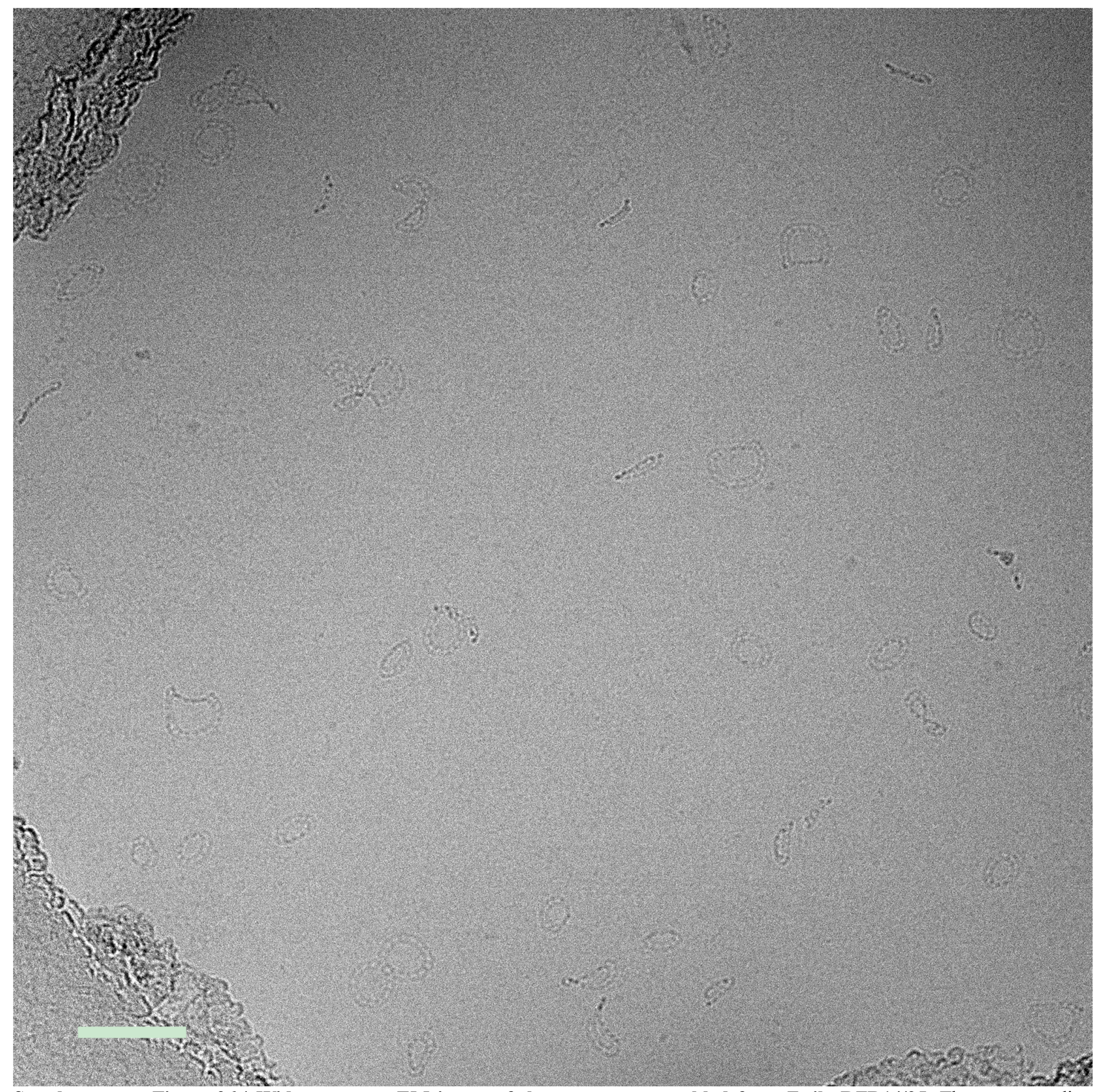

Supplementary Figure 26 | Wide-area cryo-EM image of the structure assembled from Z-tile RZB14/25. The corresponding small-area image is Figure 3c. Scale bar is $100 \mathrm{~nm}$. 


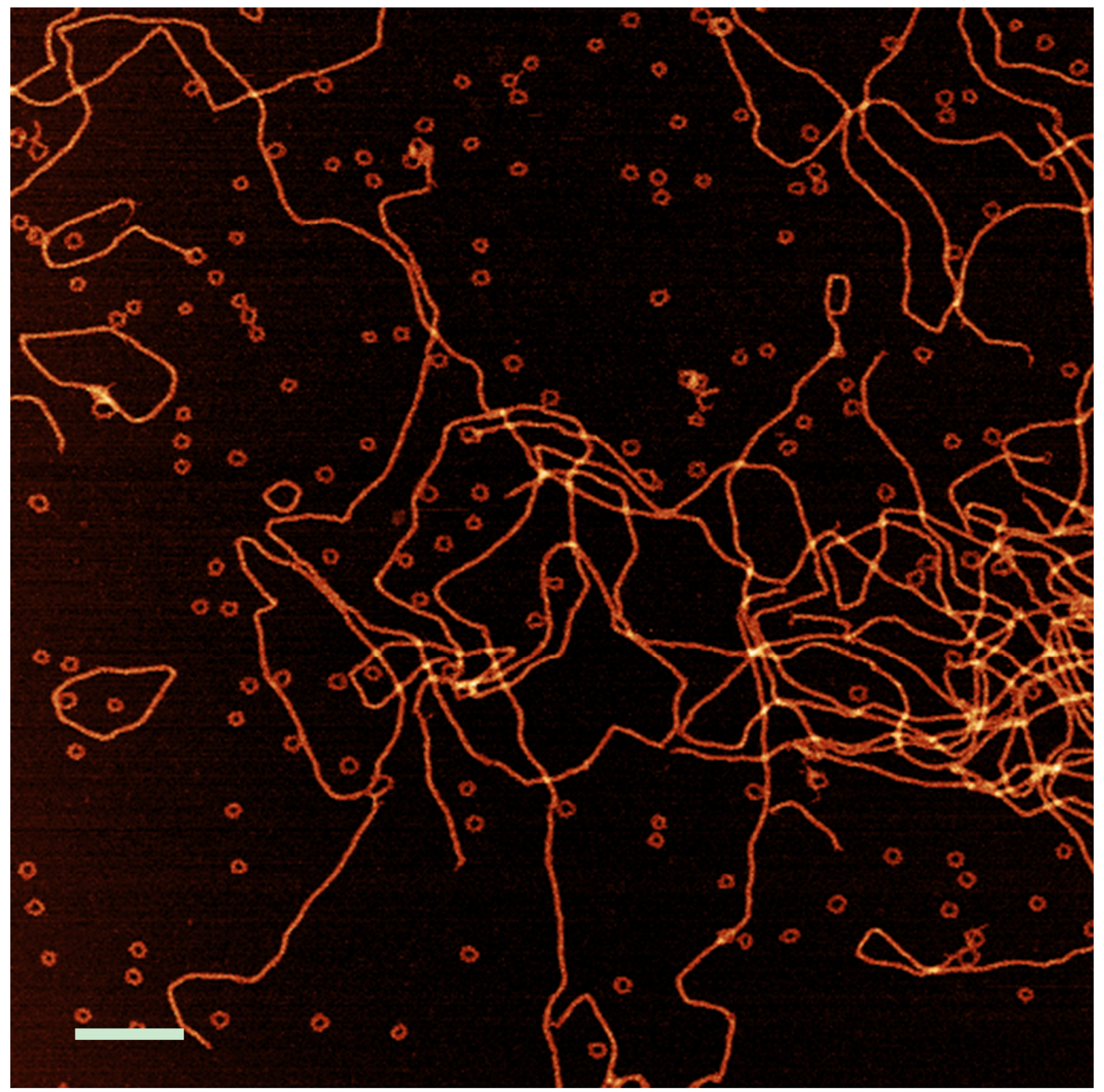

Supplementary Figure 27 | Wide-area AFM image of the structure assembled from Z-tile RZB14/36 (the sample is unfiltered). The corresponding small-area image is Figure 3e. Scale bar is $200 \mathrm{~nm}$. 


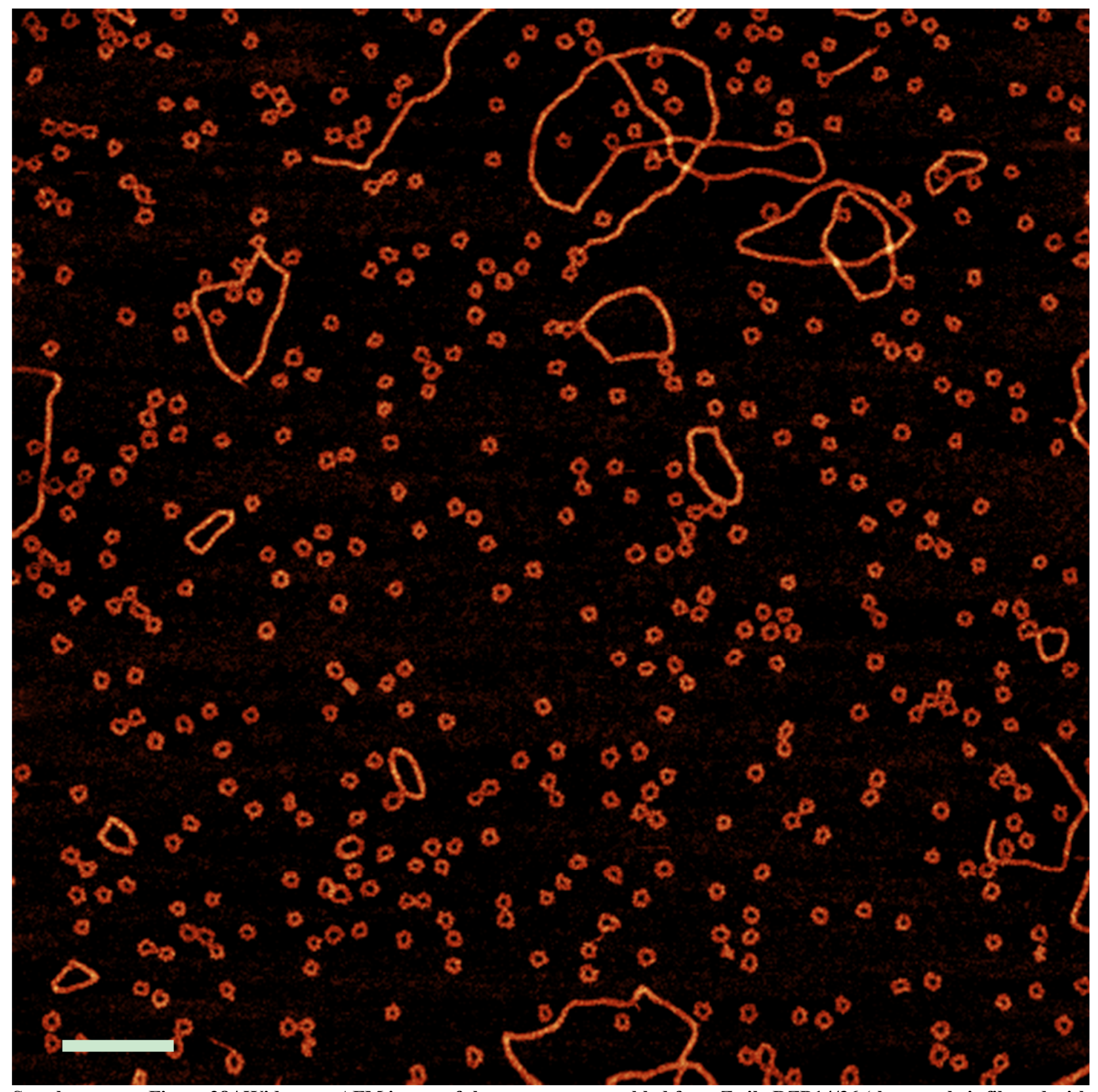

Supplementary Figure 28 | Wide-area AFM image of the structure assembled from Z-tile RZB14/36 (the sample is filtered with $0.22 \mu \mathrm{m}$ filter). The corresponding small-area image is Figure $3 \mathrm{f}$. Scale bar is $200 \mathrm{~nm}$. 


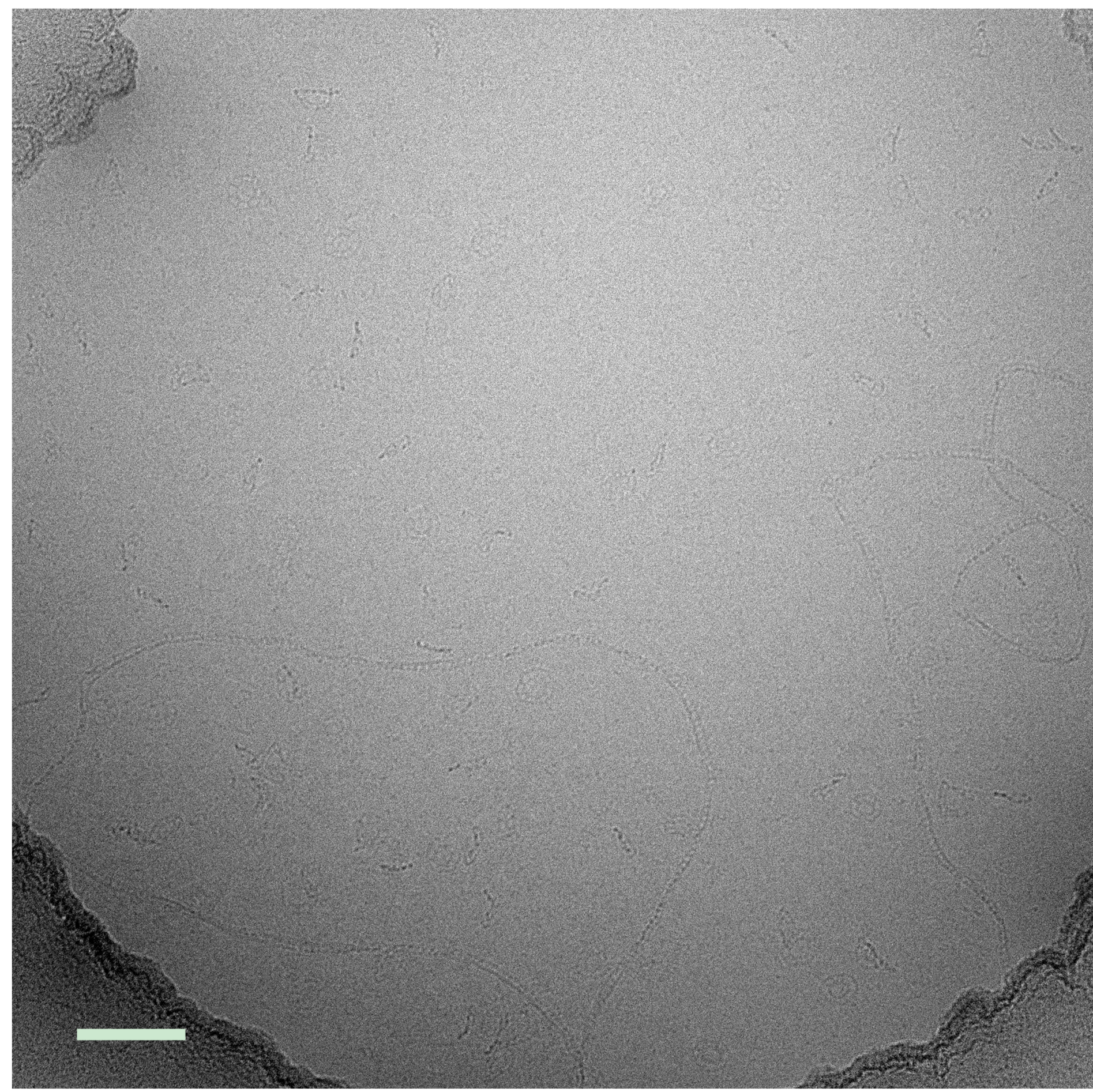

Supplementary Figure 29 | Wide-area cryo-EM image of the structure assembled from Z-tile RZB14/36 (the sample is filtered with $0.22 \mu \mathrm{m}$ filter). The corresponding small-area image is Figure $3 \mathrm{~g}$. Scale bar is $100 \mathrm{~nm}$. 


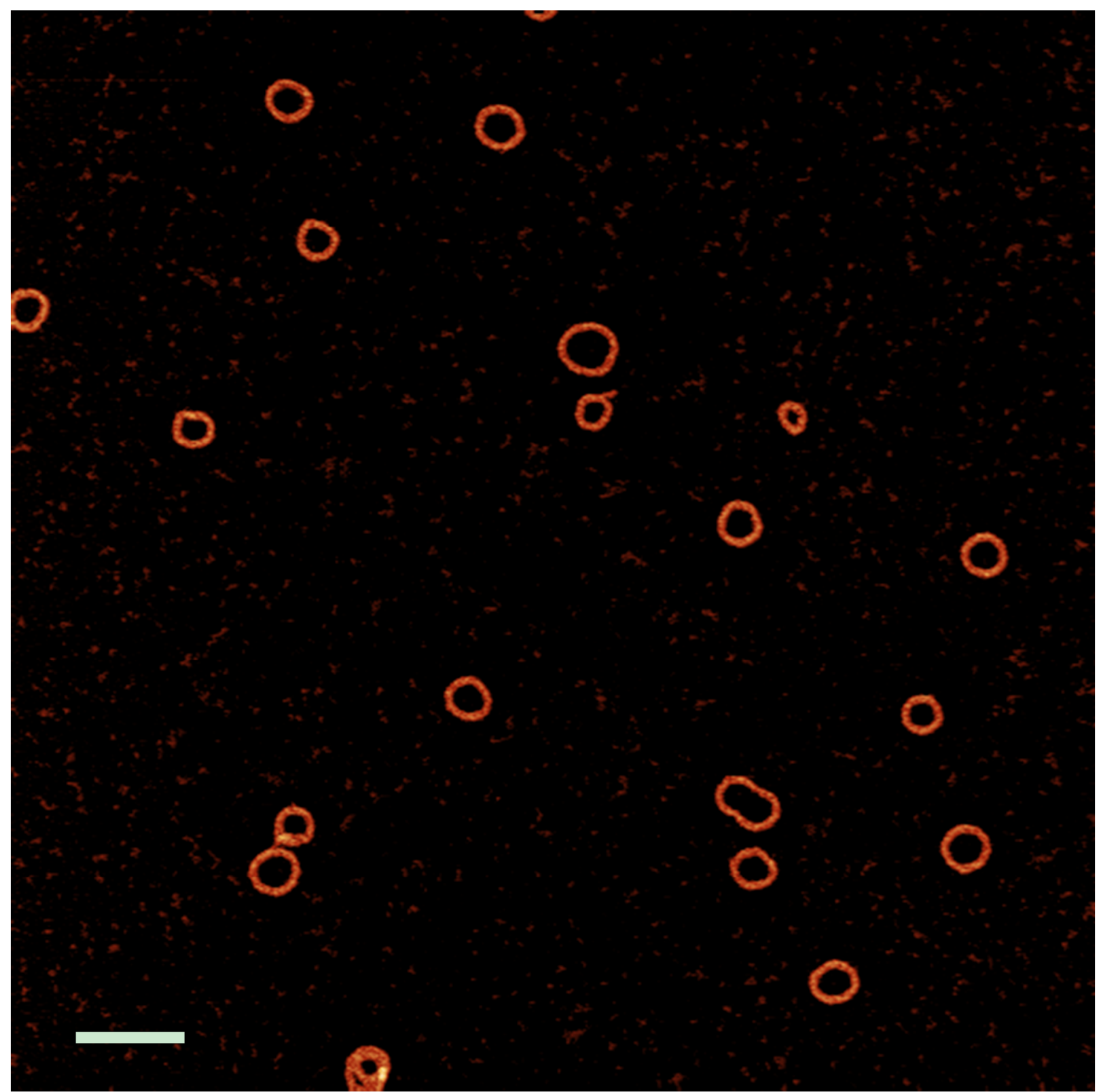

Supplementary Figure 30 | Wide-area AFM image of the structure assembled from Z-tile RZB14/47. The corresponding small-area image is Figure 3i. Scale bar is $200 \mathrm{~nm}$. 


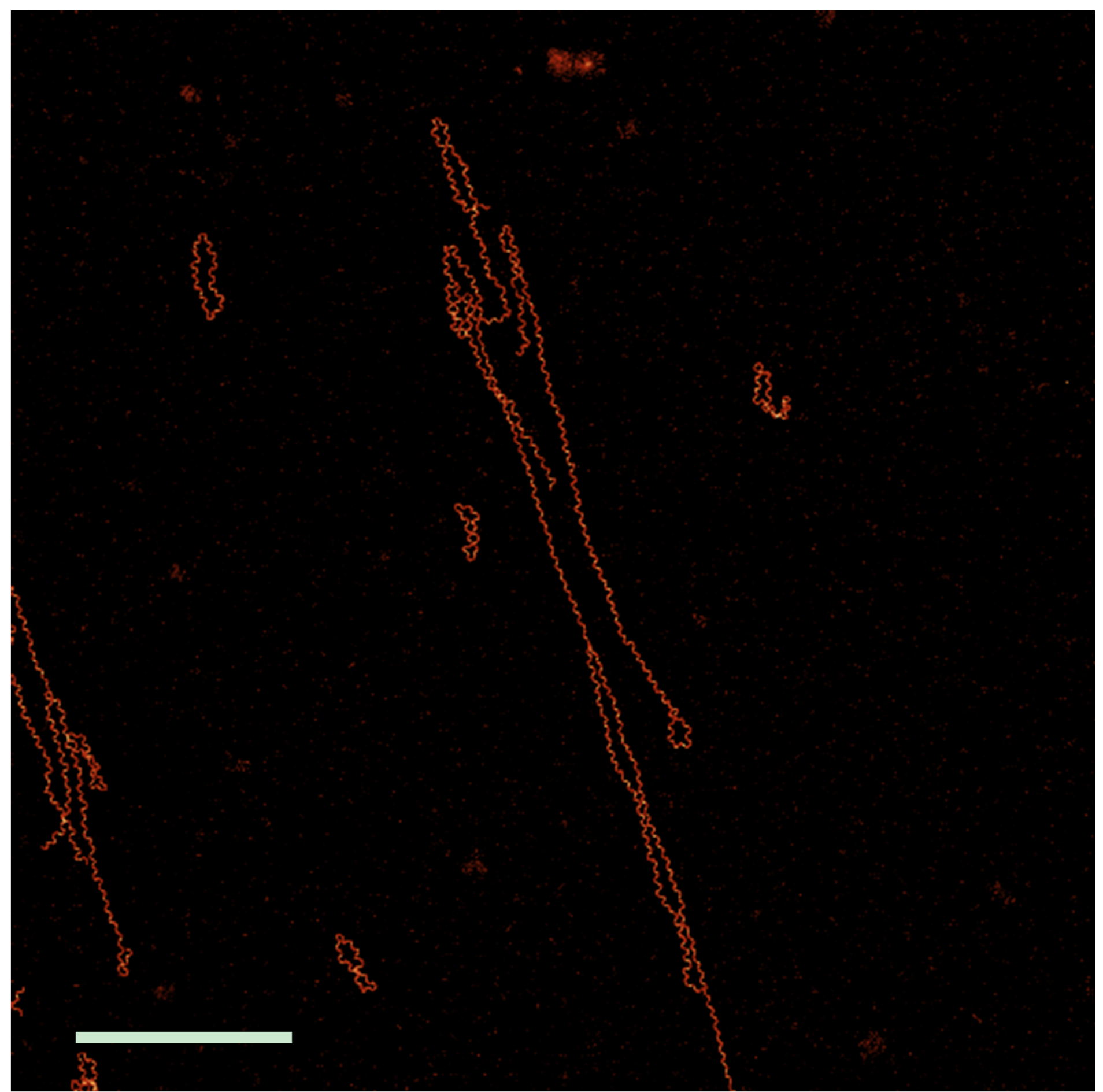

Supplementary Figure 31 | Wide-area AFM image of the structure assembled from Z-tile RZB13/24. The corresponding small-area image is Figure 3k. Scale bar is $1 \mu \mathrm{m}$. 


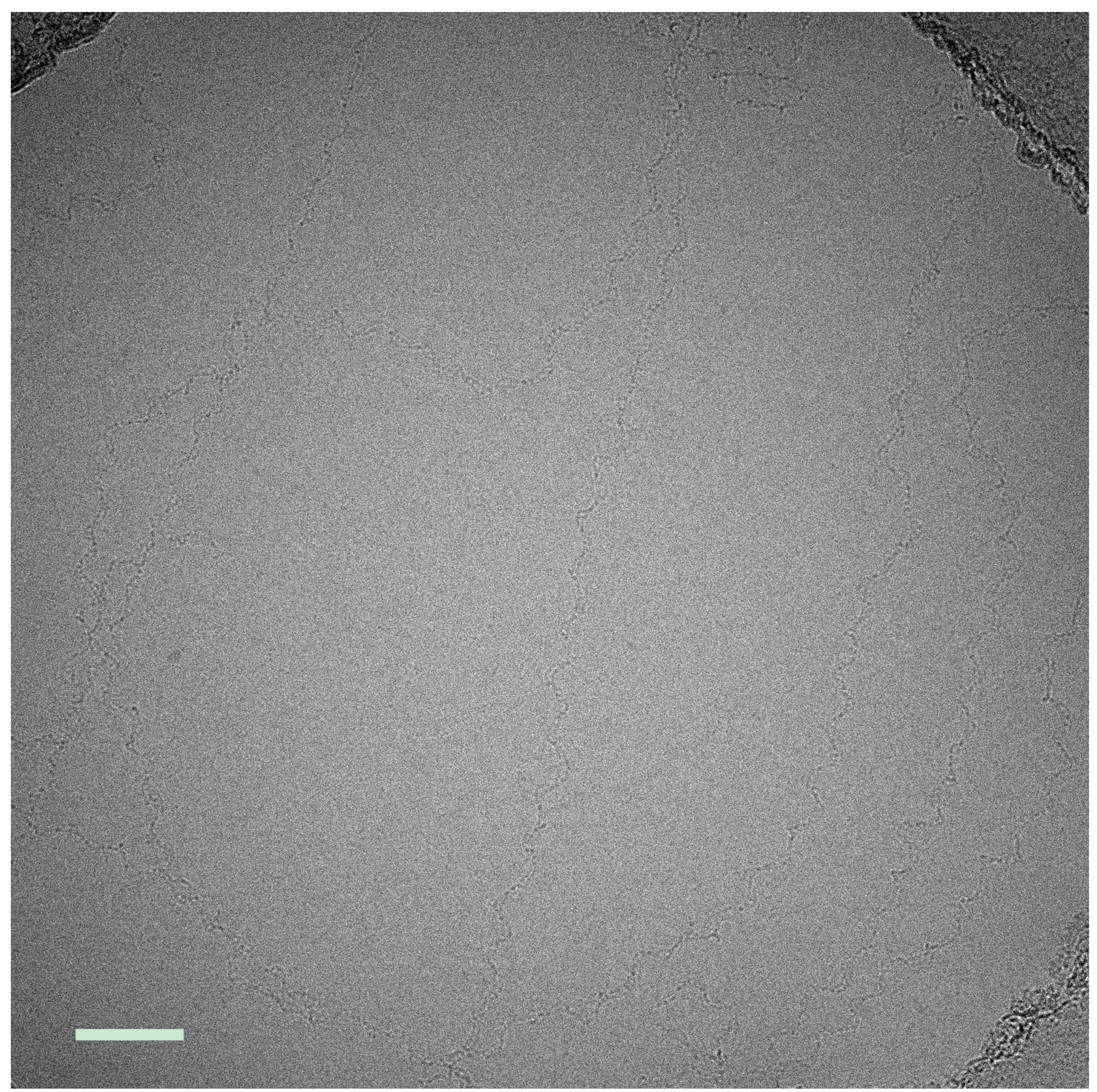

Supplementary Figure 32 | Wide-area cryo-EM image of the structure assembled from Z-tile RZB13/24. The corresponding small-area image is Figure 31. Scale bar is $100 \mathrm{~nm}$. 


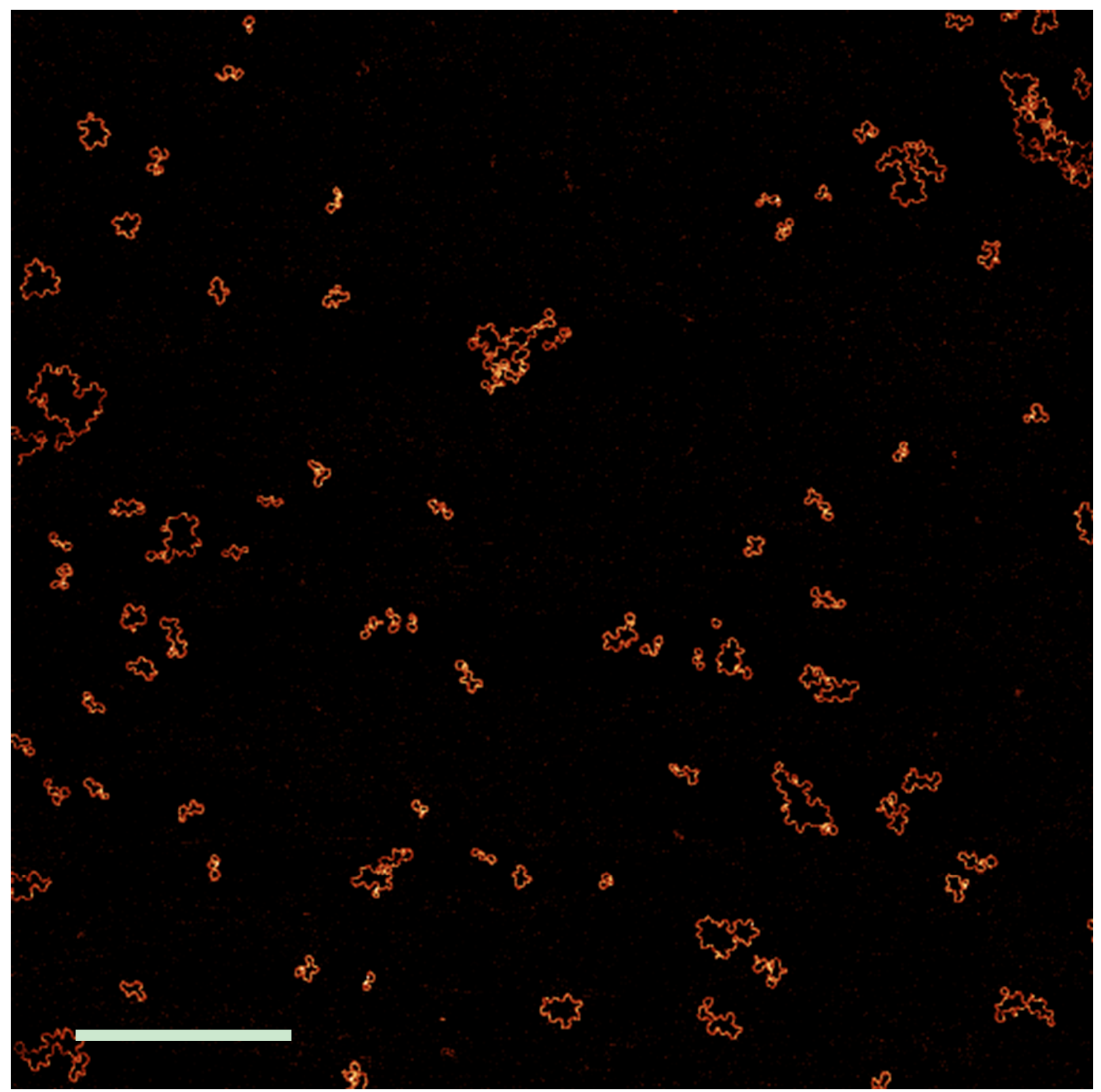

Supplementary Figure 33 | Wide-area AFM image of the structure assembled from Z-tile RZB15/26. The corresponding small-area image is Figure $3 \mathrm{~m}$. Scale bar is $1 \mu \mathrm{m}$. 


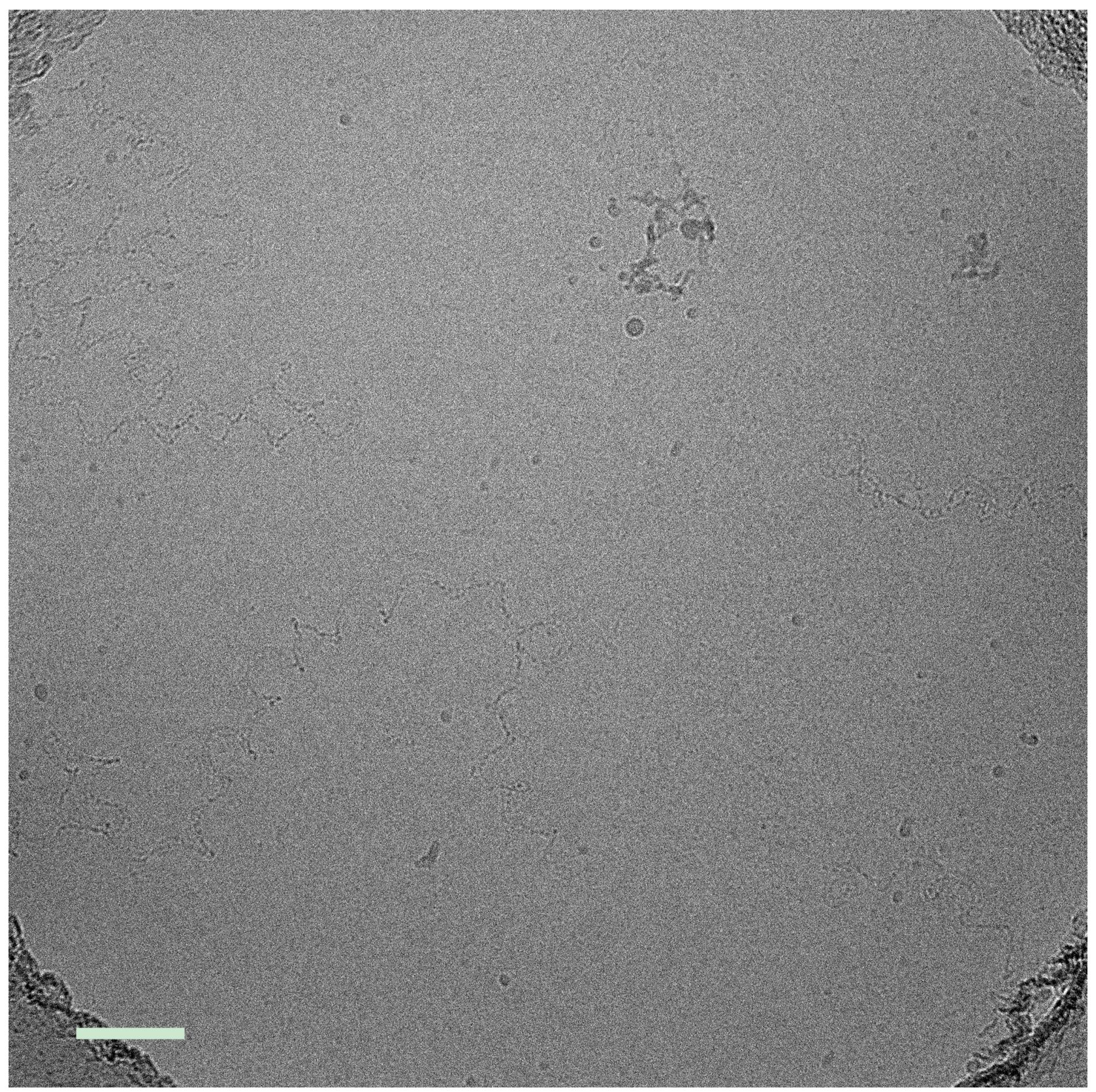

Supplementary Figure 34 | Wide-area cryo-EM image of the structure assembled from Z-tile RZB15/26. The corresponding small-area image is Figure $3 \mathrm{n}$. Scale bar is $100 \mathrm{~nm}$. 


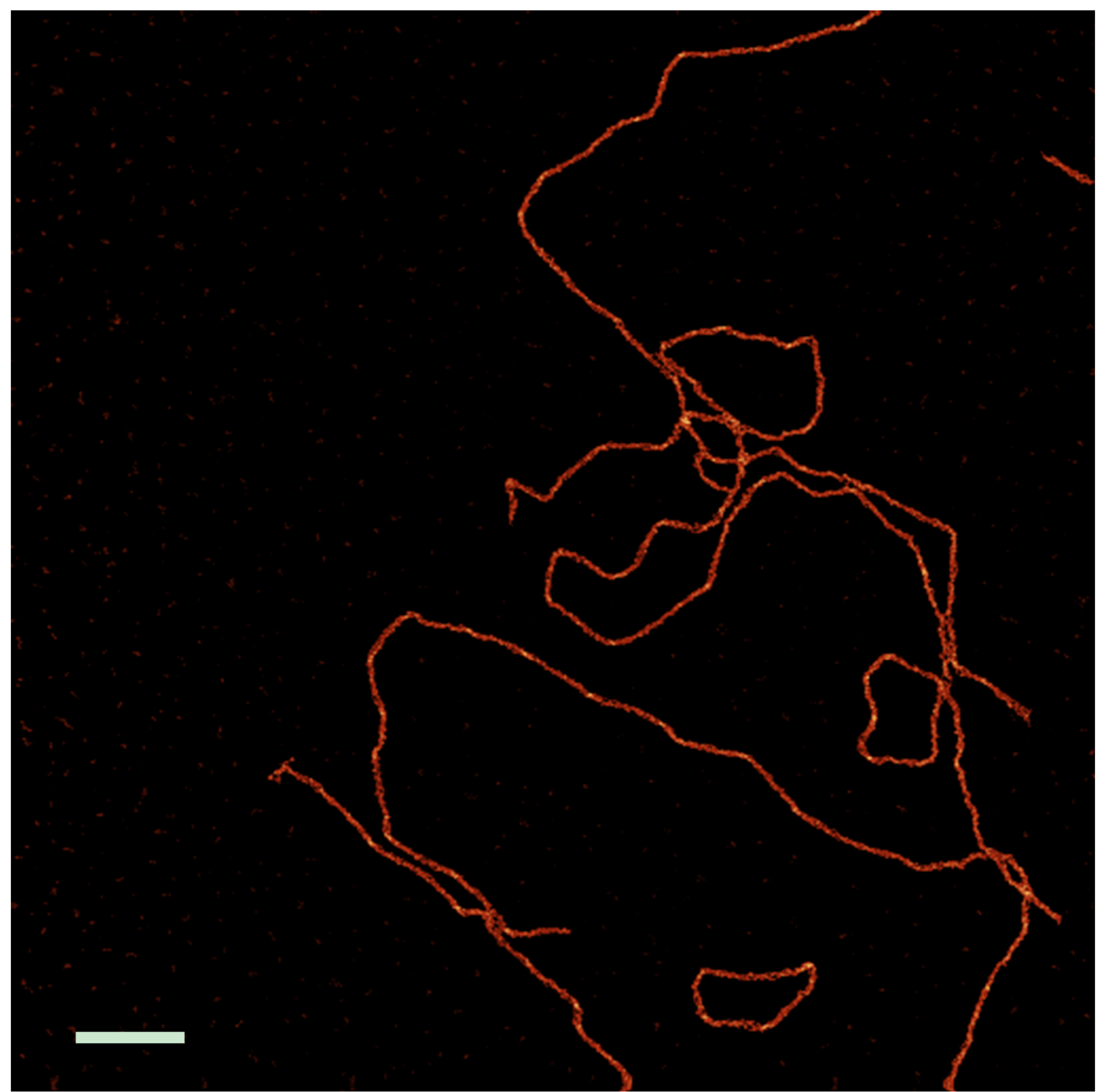

Supplementary Figure 35 | Wide-area AFM image of the structure assembled from Z-tile LZB19/47. The corresponding small-area image is Figure 4a (top right). Scale bar is $200 \mathrm{~nm}$. 


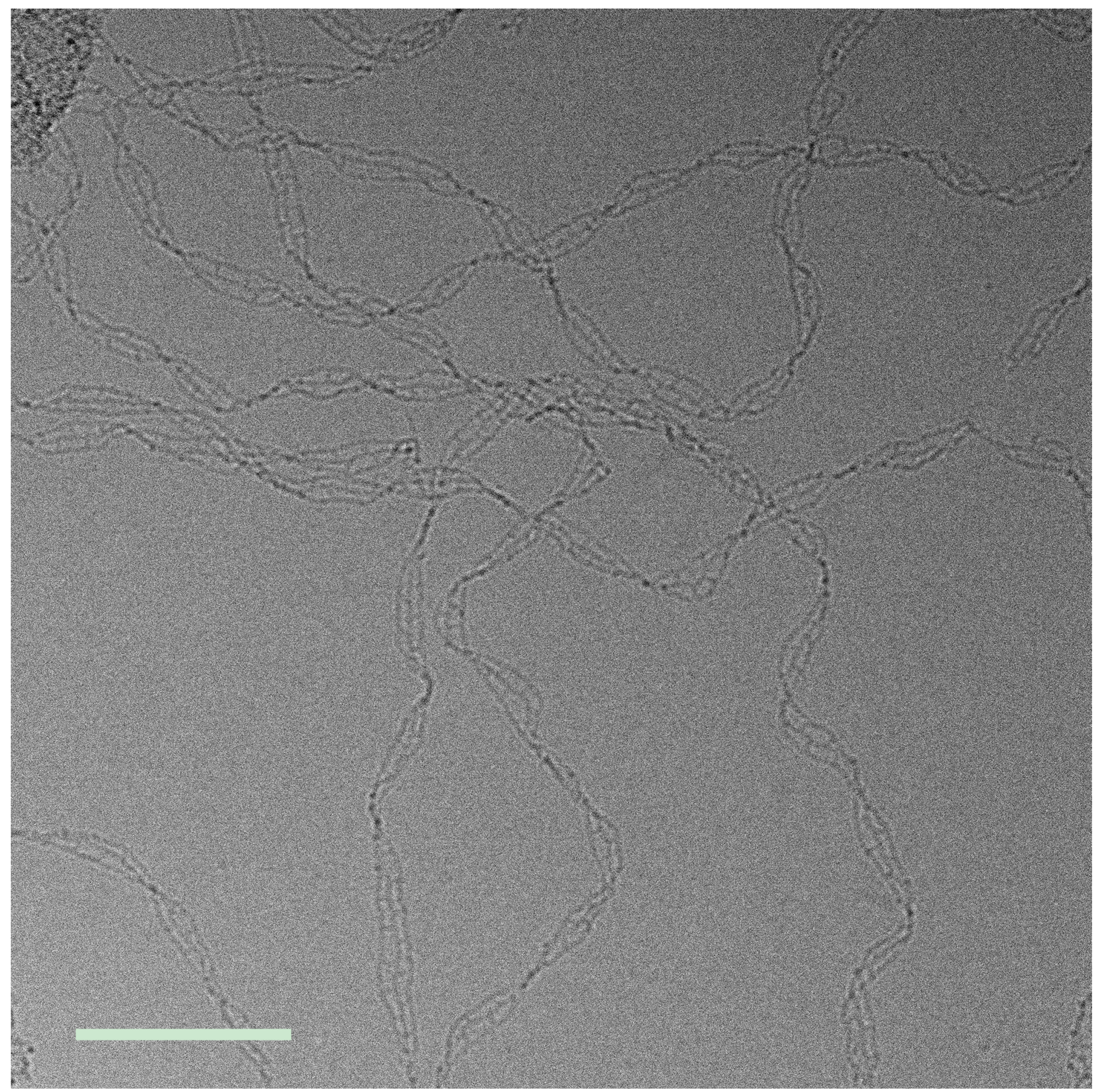

Supplementary Figure 36 | Wide-area cryo-EM image of the structure assembled from Z-tile LZB19/47. The corresponding small-area image is the bottom right panel in Figure 4a. Scale bar is $100 \mathrm{~nm}$. 


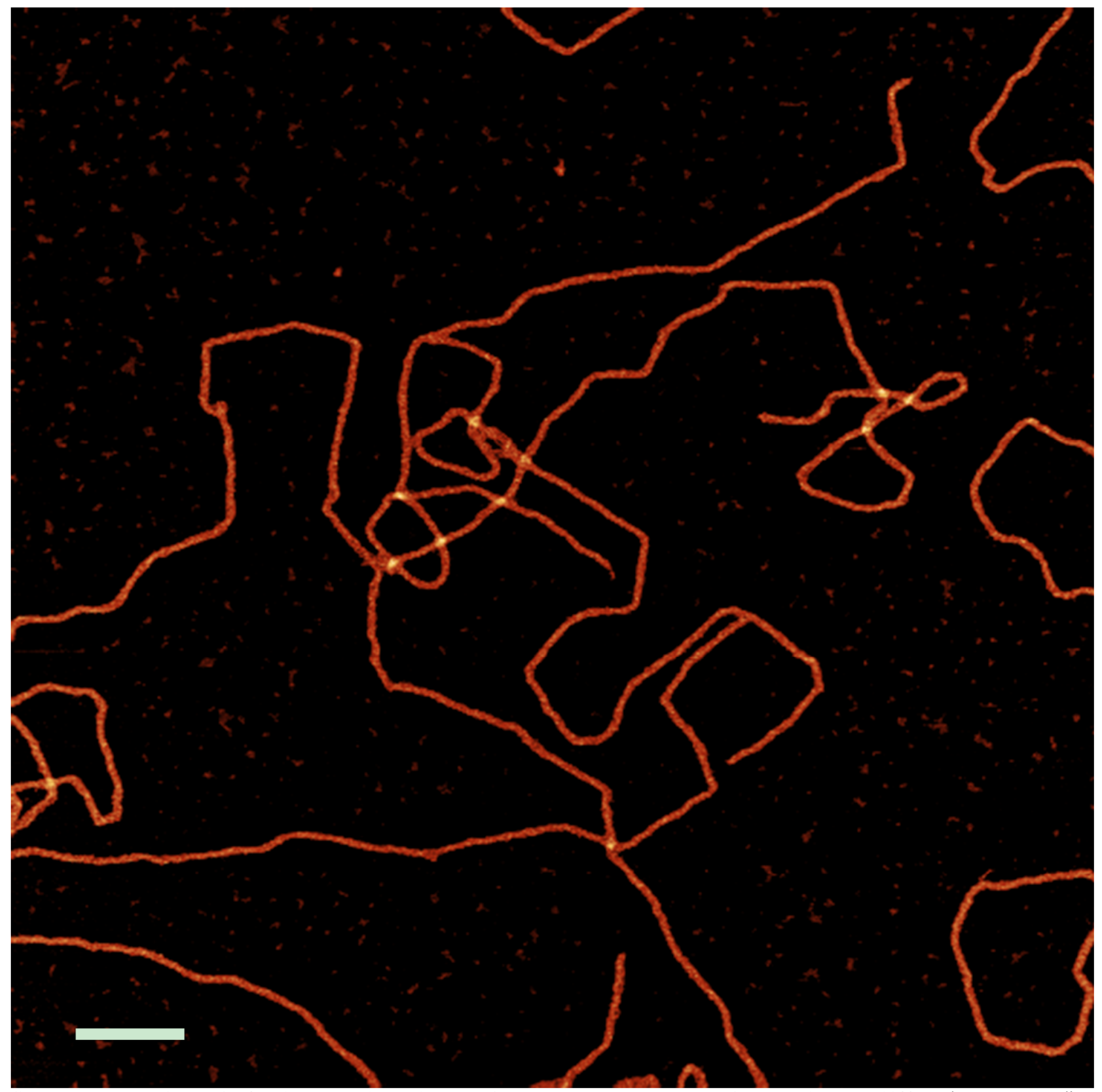

Supplementary Figure 37 | Wide-area AFM image of the structure assembled from Z-tile LZB10/47. The corresponding small-area image is the top right panel in Figure 4b. Scale bar is $200 \mathrm{~nm}$. 


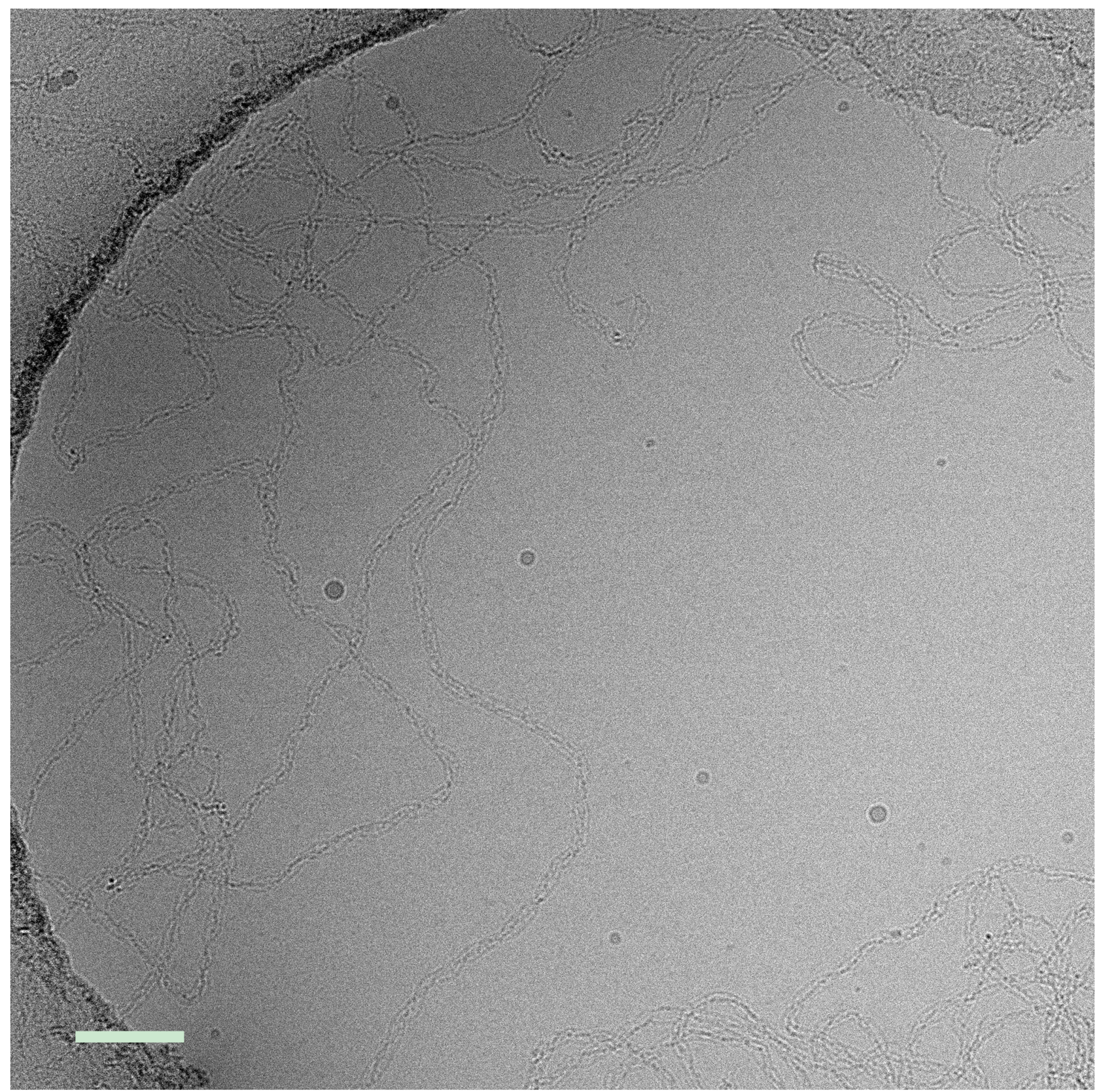

Supplementary Figure 38 | Wide-area cryo-EM image of the structure assembled from Z-tile LZB10/47. The corresponding small-area image is the bottom right panel in Figure 4b. Scale bar is $100 \mathrm{~nm}$. 


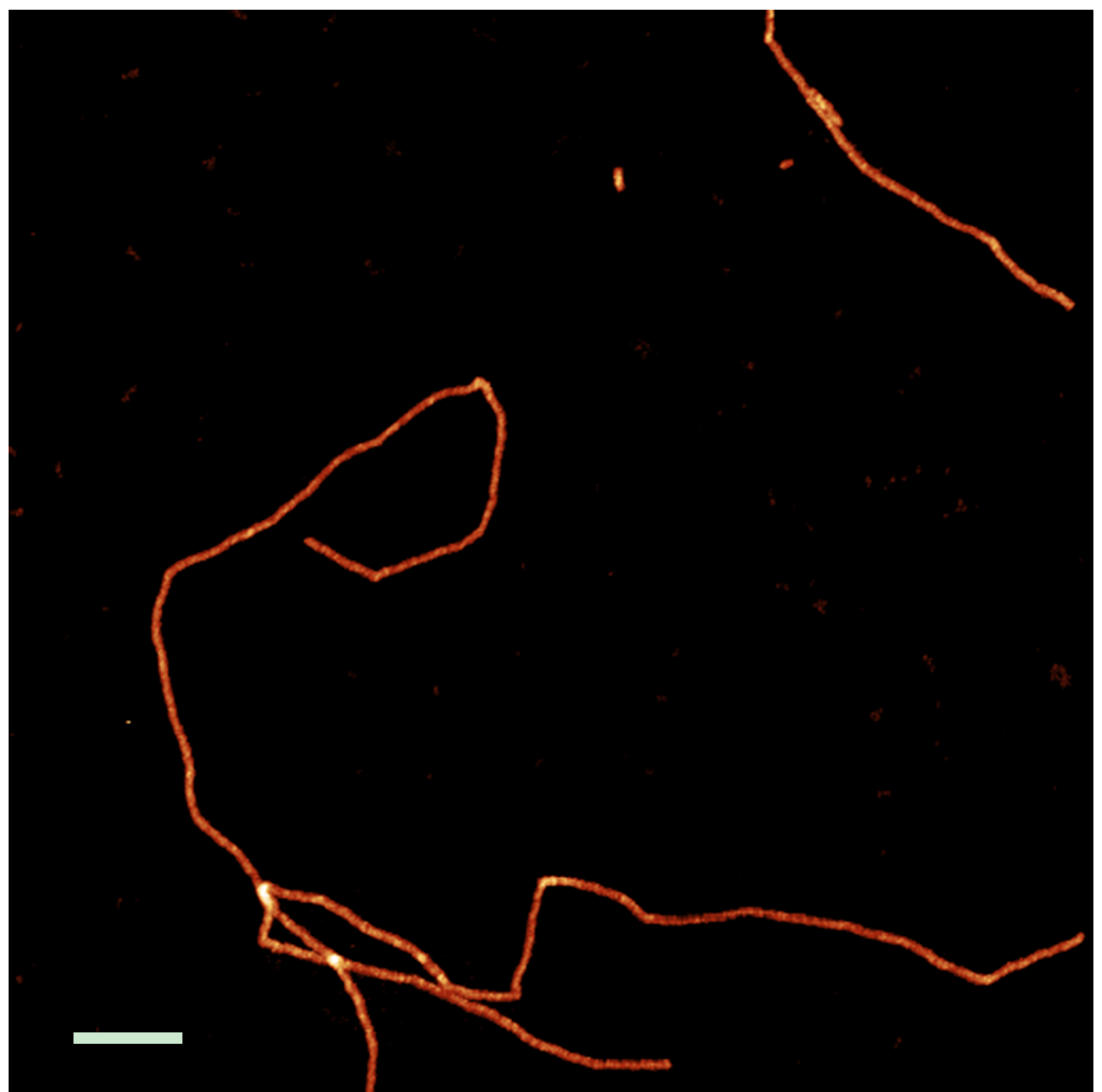

Supplementary Figure 39 | Wide-area AFM image of the structure assembled from claw-like tile LCS3WJ. The corresponding small-area image is the middle panel in Figure 4c. Scale bar is $200 \mathrm{~nm}$. 


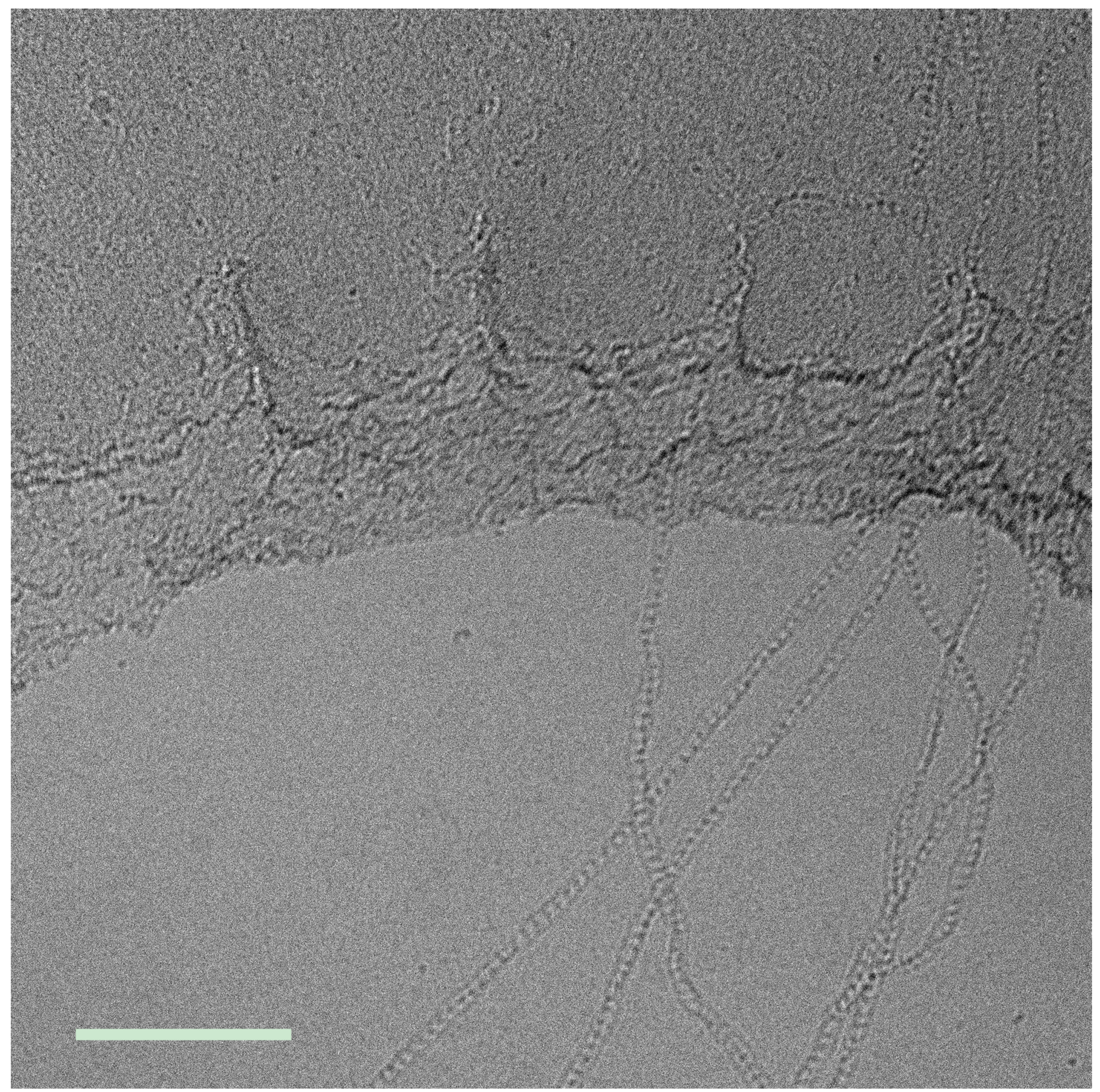

Supplementary Figure 40| Wide-area cryo-EM image of the structure assembled from claw-like tile LCS3WJ. The corresponding small-area image is the right panel in Figure 4c. Scale bar is $100 \mathrm{~nm}$. 




Supplementary Figure 41 | Wide-area AFM image of the structure assembled from claw-like tile LCS4WJ. The corresponding small-area image is the middle panel in Figure 4d. Scale bar is $200 \mathrm{~nm}$. 


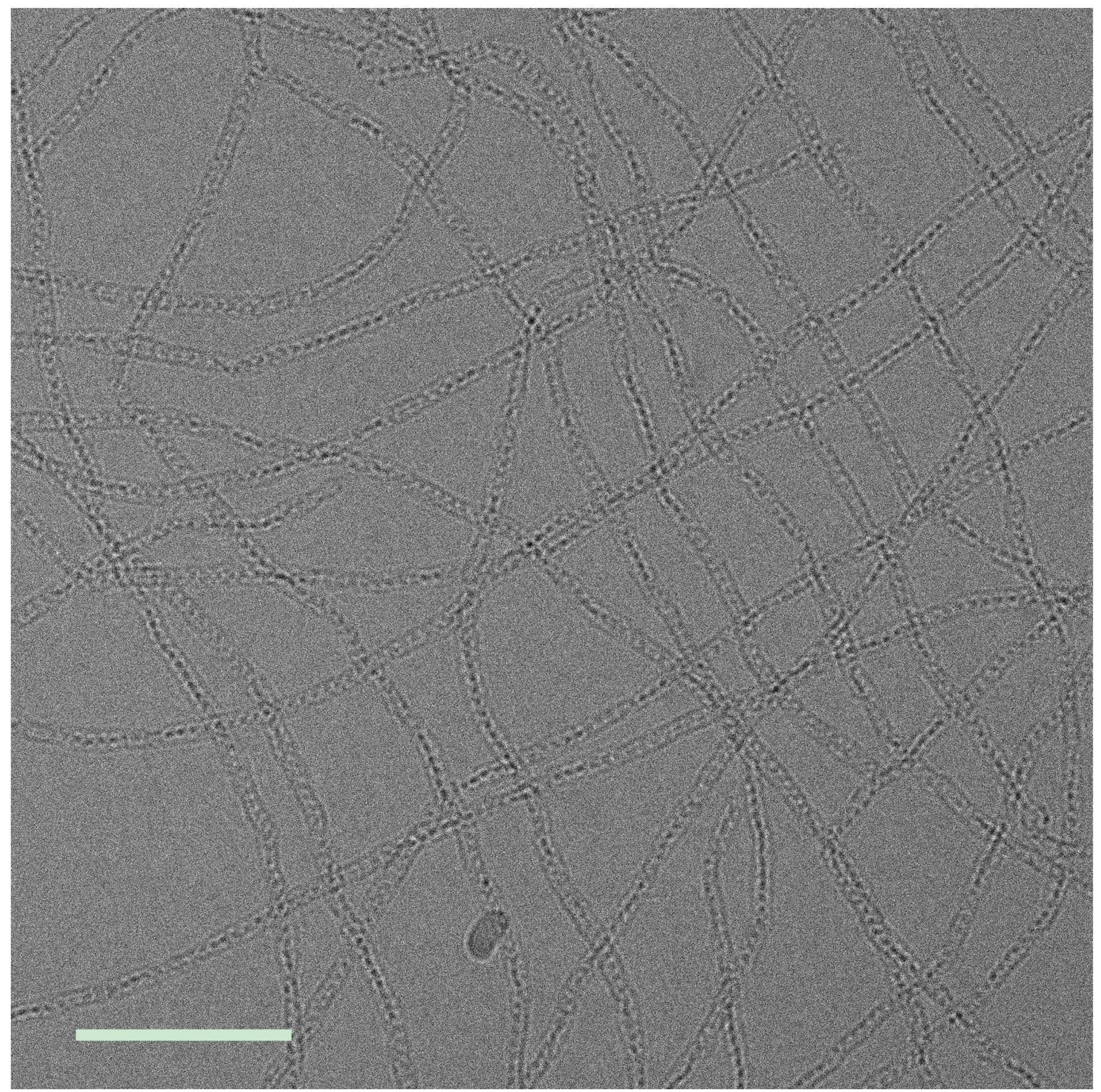

Supplementary Figure 42 | Wide-area cryo-EM image of the structure assembled from claw-like tile LCS4WJ. The corresponding small-area image is the right panel in Figure 4d. Scale bar is $100 \mathrm{~nm}$. 


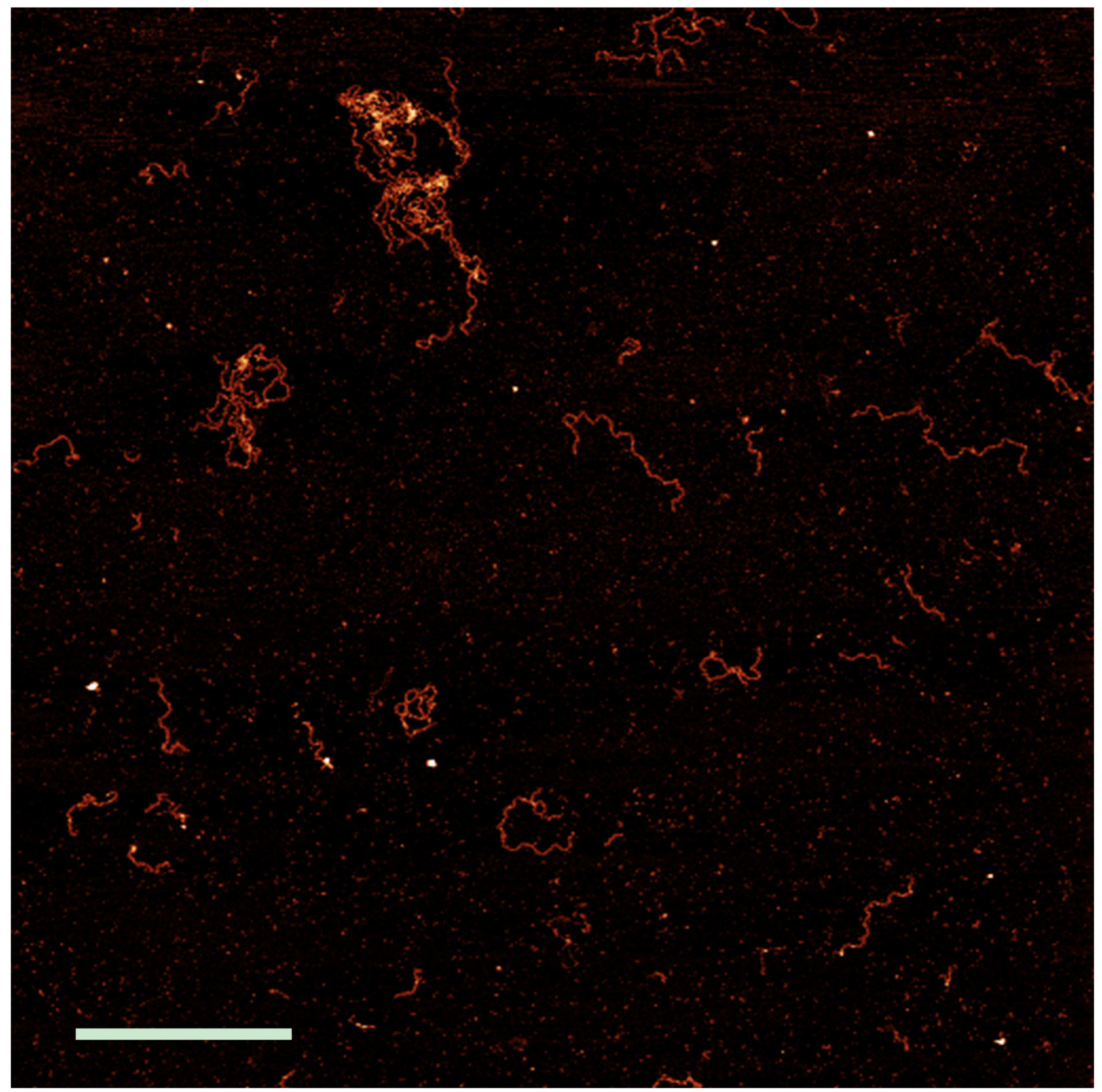

Supplementary Figure 43 | Wide-area AFM image of the structure assembled in vitro from TSSP that is produced in $E$. coli. and purified by dPAGE. The corresponding small-area image is Figure 6f. Scale bar is $1 \mu \mathrm{m}$. 


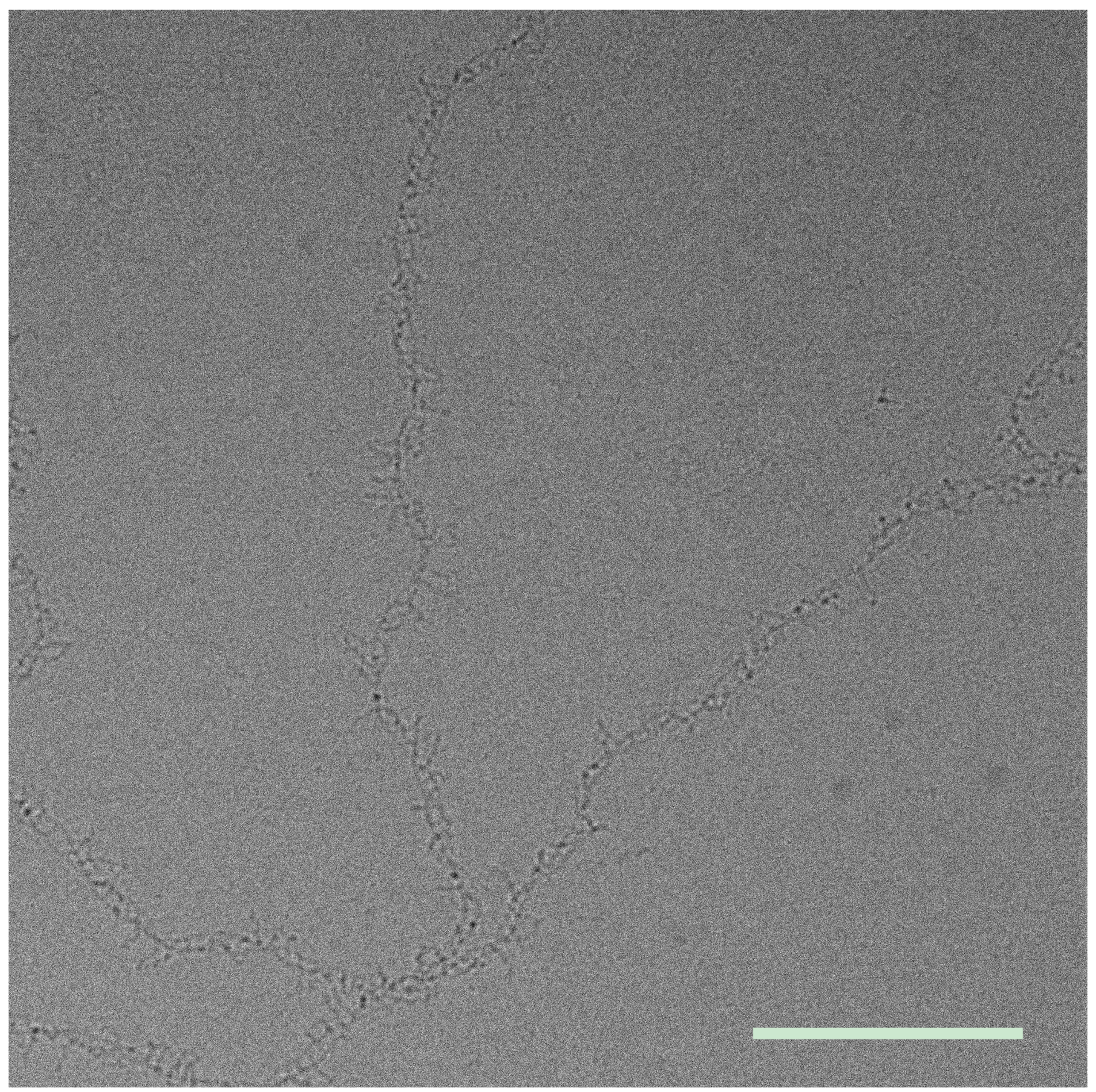

Supplementary Figure 44 | Wide-area cryo-EM image of the structure assembled in vitro from TSSP that is produced in $E$. coli. and purified by dPAGE. The corresponding small-area image is Figure $6 \mathrm{~g}$. Scale bar is $100 \mathrm{~nm}$. 


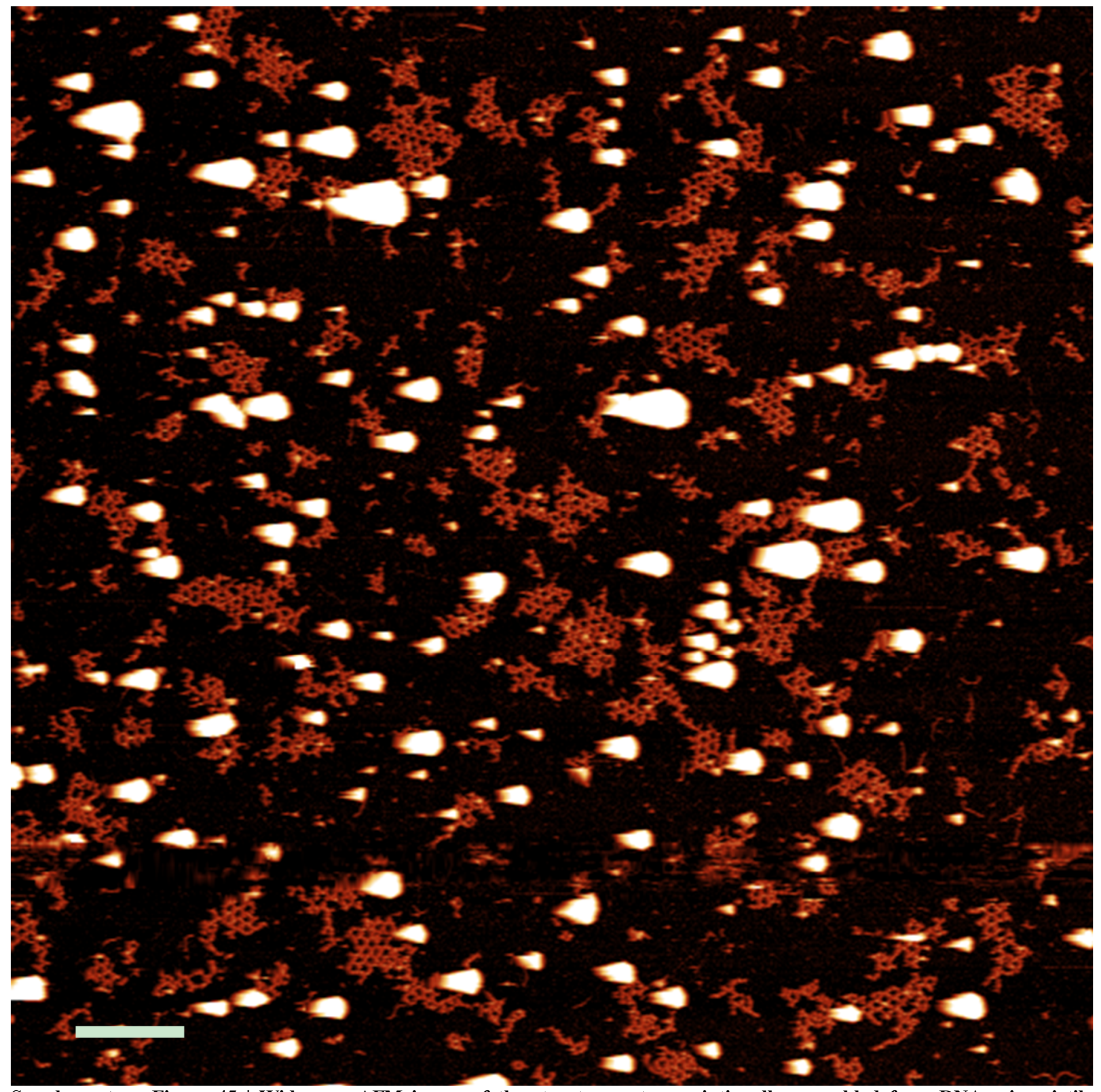

Supplementary Figure 45 | Wide-area AFM image of the structure cotranscriptionally assembled from RNA origami tile DAE-bKL. The corresponding small-area image is Figure 7c. Scale bar is $200 \mathrm{~nm}$. 


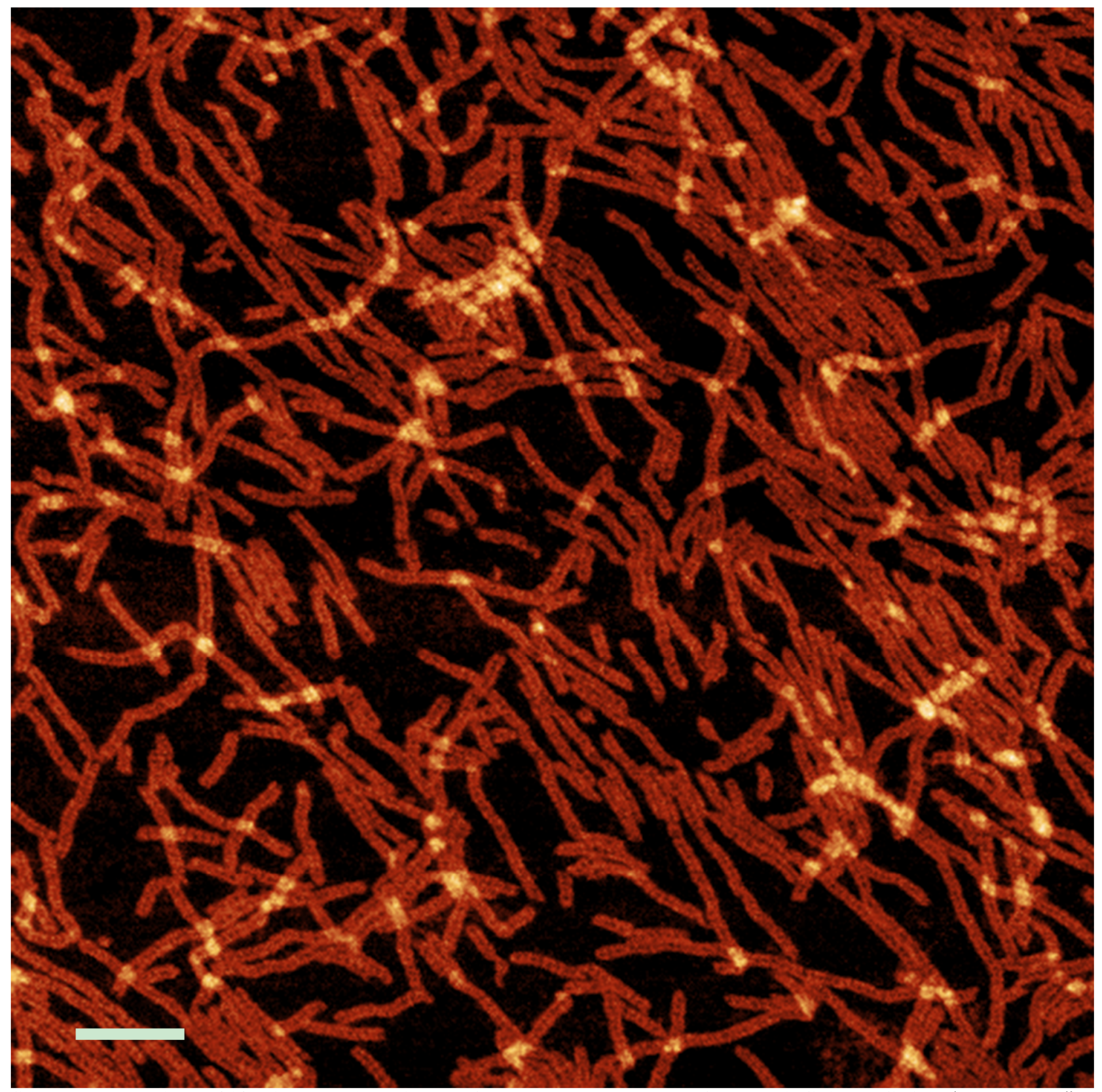

Supplementary Figure 46 | Wide-area AFM image of the structure assembled from DNA C-tile dLCB15. The corresponding small-area image is Figure $7 \mathrm{f}$. Scale bar is $100 \mathrm{~nm}$. 
